Supporting Information

\title{
Palladium-Catalyzed Annulation of Internal Alkynes: Direct Access to $\pi$-Conjugated Ullazines
}

Danyang Wan, Xiaoyu Li, Ruyong Jiang, Boya Feng, Jingbo Lan, Ruilin Wang and Jingsong You*

Key Laboratory of Green Chemistry and Technology of Ministry of Education, College of Chemistry, and State Key Laboratory of Biotherapy, West China Medical School, Sichuan University, 29 Wangjiang Road, Chengdu 610064, PR China College of Materials Science and Engineering, Sichuan University, 29 Wangjiang Road, Chengdu 610064, PR China 


\section{Table of Contents}

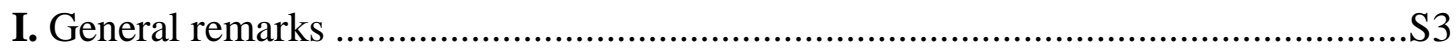

II. Optimization of Lewis acid additives used in the annulation reaction ..................S3

III. General procedure for the synthesis of 2,6-dibromophenylpyrroles ....................S5

IV. General procedure for the Pd-catalyzed cyclization reaction of

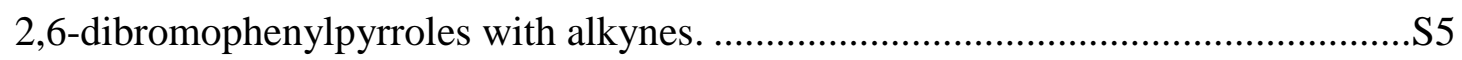

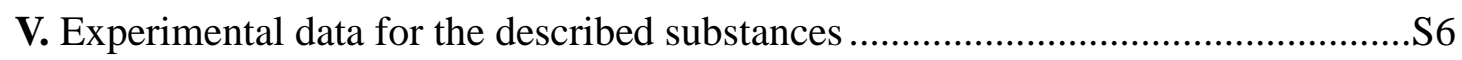

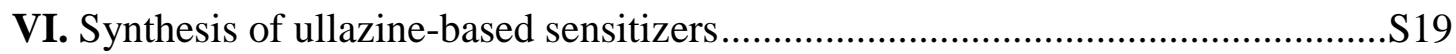

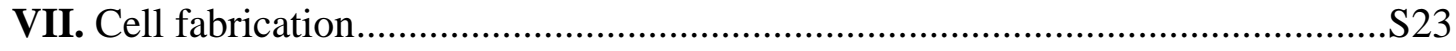

VIII. Electrochemical and photochemical properties of dyes ..............................S23

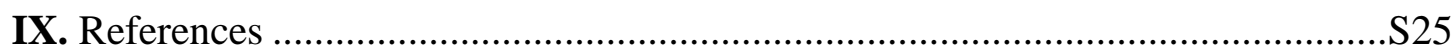

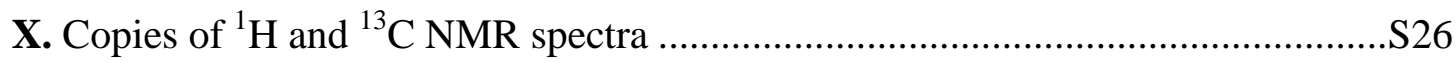




\section{General remarks}

NMR spectra were obtained on a Bruker AV II-400 MHz spectrometer. The ${ }^{1} \mathrm{H}$ NMR (400 MHz) chemical shifts and the ${ }^{13} \mathrm{C}$ NMR (100 MHz) chemical shifts were measured relative to $\mathrm{CDCl}_{3}$ as the internal reference. High resolution mass spectra (HRMS) were obtained with a Waters-Q-TOF-Premier (ESI) or a Shimadzu LCMS-IT-TOF (ESI). Melting points were determined with XRC-1 and are uncorrected. UV/vis spectra were measured on a HITACHI U-2910. Fluorescence spectra were collected on a Horiba Jobin Yvon-Edison Fluoromax-4 fluorescence spectrometer with a calibrated integrating sphere system.

Unless otherwise noted, all reagents were obtained from commercial suppliers and used without further purification. The dye bis(tetrabutylammonium)-cis-di(thiocyanato)- $N, N^{\prime}$-bis(4-carboxylato-4'-carboxylic acid-2,2'-bipyridine)ruthenium (coded as N719) and Pt-counter electrode were purchased from OPV Tech (China) Co., Ltd.. Two types of $\mathrm{TiO}_{2}$ photoanodes were purchased from OPV Tech (China) Co., Ltd.: 1) $7 \mu \mathrm{m}$ of $20 \mathrm{~nm} \mathrm{TiO}_{2}$ particles as the absorbing layer with dimensions of $1.0 \times 1.0 \mathrm{~cm}^{2}$; 2) $13 \mu \mathrm{m}$ of $20 \mathrm{~nm} \mathrm{TiO}{ }_{2}$ particles as the absorbing layer and $5 \mu \mathrm{m}$ of $400 \mathrm{~nm} \mathrm{TiO}_{2}$ particles as the scattering layer with dimensions of $0.4 \times 0.4 \mathrm{~cm}^{2}$. Both of them are coated on an FTO glass substrate by screen printing, and the film thickness was measured by means of a profilometer (E-MC-S24B., Japan). The $7 \mu \mathrm{m} \mathrm{TiO}_{2}$ thin film was dipped into a solution containing $5.0 \times 10^{-4} \mathrm{M}$ of the dye in chloroform/ethanol (4:6, v/v) for $24 \mathrm{~h}$ for UV-Vis absorption spectra test. The $0.4 \times 0.4 \mathrm{~cm}^{2} \mathrm{TiO}_{2}$ thin film $(13 \mu \mathrm{m}$ of $20 \mathrm{~nm}$ particles as the absorbing layer and $5 \mu \mathrm{m}$ of $400 \mathrm{~nm}$ particles as the scattering layer) was used for device fabrication. Alkynes ${ }^{1}$, 1-(2,6-dibromophenyl)-1H-pyrrole ${ }^{2}$, and 2,6-dibromoanilines ${ }^{3}$ were prepared according to the literature procedure. Solvents were dried by refluxing over $\mathrm{CaH}_{2}$ (acetonitrile) and freshly distilled prior to use. Unless otherwise indicated, all reactions were carried out under $\mathrm{N}_{2}$ atmosphere.

\section{Optimization of Lewis acid additives used in the annulation reaction}

A flame-dried Schlenk tube with a magnetic stir bar was charged with 
1-(2,6-dibromophenyl)-1H-pyrrole (75.2 mg, $0.25 \mathrm{mmol})$, diphenyl acetylene (133.7 $\mathrm{mg}, 0.75 \mathrm{mmol}), \mathrm{K}_{2} \mathrm{CO}_{3}(103.7 \mathrm{mg}, 0.75 \mathrm{mmol})$, catalyst $(50.0 \mu \mathrm{mol})$, ligand (0.10 $\mathrm{mmol})$, additive $(0.50 \mathrm{mmol})$ and $\mathrm{MeCN}(2.0 \mathrm{~mL})$ under $\mathrm{N}_{2}$. The tube was sealed with a teflon-coated screw cap and the reaction solution was heated at $120{ }^{\circ} \mathrm{C}$ for $24 \mathrm{~h}$. The mixture was then cooled to ambient temperature, diluted with $10 \mathrm{~mL}$ of $\mathrm{CH}_{2} \mathrm{Cl}_{2}$, filtered through a celite pad, and washed with $10-20 \mathrm{~mL}$ of $\mathrm{CH}_{2} \mathrm{Cl}_{2}$. The combined organic phases were concentrated and the residue was purified by column chromatography on silica gel to provide the desired product.

Table S1. Optimization of Lewis Acid Additives Used in the Annulation Reaction ${ }^{a}$

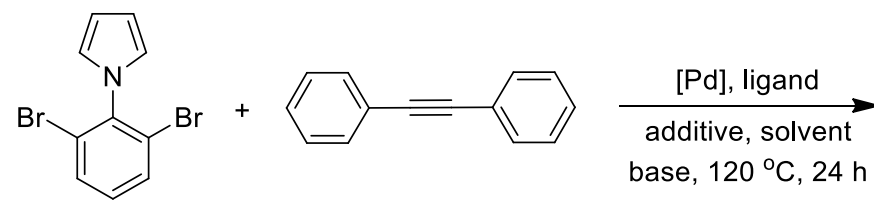

$1 \mathbf{a}$

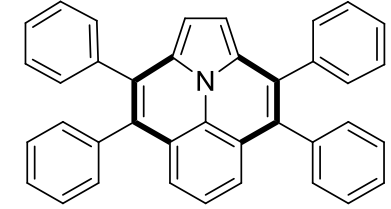

3aa

$\begin{array}{ccccc}\text { entry } & \text { catalyst }^{b} & \text { additive } & \text { base } & \text { yield }(\%) \\ 1 & \mathrm{Pd}(\mathrm{TFA})_{2}, \mathbf{L} \mathbf{1} & \mathrm{LiCl} & \mathrm{K}_{2} \mathrm{CO}_{3} & \text { trace } \\ 2 & \mathrm{Pd}(\mathrm{TFA})_{2}, \mathbf{L 2} & \mathrm{LiCl} & \mathrm{K}_{2} \mathrm{CO}_{3} & \text { trace } \\ 3 & \mathrm{Pd}(\mathrm{TFA})_{2}, \mathbf{L 3} & \mathrm{LiCl} & \mathrm{K}_{2} \mathrm{CO}_{3} & \text { trace } \\ 4 & \mathrm{Pd}(\mathrm{TFA})_{2}, \mathbf{L} \mathbf{4} & \mathrm{LiCl} & \mathrm{K}_{2} \mathrm{CO}_{3} & 12 \\ 5 & \mathrm{Pd}(\mathrm{TFA})_{2}, \mathbf{L 5} & \mathrm{LiCl} & \mathrm{K}_{2} \mathrm{CO}_{3} & \text { trace } \\ 6 & \mathrm{Pd}(\mathrm{TFA})_{2}, \mathbf{L 6} & \mathrm{LiCl} & \mathrm{K}_{2} \mathrm{CO}_{3} & \text { trace } \\ 7 & \mathrm{Pd}(\mathrm{TFA})_{2}, \mathbf{L 7} & \mathrm{LiCl} & \mathrm{K}_{2} \mathrm{CO}_{3} & 15 \\ 8 & \mathrm{Pd}(\mathrm{TFA})_{2}, \mathbf{L 8} & \mathrm{LiCl} & \mathrm{K}_{2} \mathrm{CO}_{3} & \text { trace } \\ 9 & \mathrm{Pd}(\mathrm{TFA})_{2}, \mathbf{L 9} & \mathrm{Cu}(\mathrm{OTf})_{2} & \mathrm{~K}_{2} \mathrm{CO}_{3} & 30 \\ 10 & \mathrm{Pd}(\mathrm{TFA})_{2}, \mathbf{L 9} & \mathrm{AgOTf} & \mathrm{K}_{2} \mathrm{CO}_{3} & \text { trace } \\ 11 & \mathrm{Pd}(\mathrm{TFA})_{2}, \mathbf{L 9} & \mathrm{Sc}(\mathrm{OTf})_{3} & \mathrm{~K}_{2} \mathrm{CO}_{3} & 60 \\ 12 & \mathrm{Pd}(\mathrm{TFA})_{2}, \mathbf{L 9} & \mathrm{Mn}(\mathrm{OAc})_{3} & \mathrm{~K}_{2} \mathrm{CO}_{3} & \text { trace } \\ 13 & \mathrm{Pd}(\mathrm{TFA})_{2}, \mathbf{L 9} & \mathrm{InBr} & \mathrm{K}_{2} \mathrm{CO}_{3} & \text { trace } \\ 14 & \mathrm{Pd}(\mathrm{TFA})_{2}, \mathbf{L 9} & \mathrm{AgOAc} & \mathrm{K}_{2} \mathrm{CO}_{3} & \text { trace }\end{array}$

${ }^{a}$ The reaction was performed with $\mathbf{1 a}(0.25 \mathrm{mmol}), \mathbf{2 a}(0.75 \mathrm{mmol}, 3.0$ equiv), catalyst $(0.05$ 
mmol), ligand $(0.10 \mathrm{mmol})$, additive $(0.50 \mathrm{mmol})$, base $(0.75 \mathrm{mmol})$ in $2.0 \mathrm{~mL}$ of $\mathrm{MeCN}$ under a $\mathrm{N}_{2}$ atmosphere. Isolated yield. ${ }^{b} \mathbf{L 1}=2$-(di-tert-butylphosphino)biphenyl, $\mathbf{L} \mathbf{2}=$ tris(2-methoxyphenyl)phosphine, $\mathbf{L 3}=$ butyldi-1-adamantylphosphine, $\mathbf{L 4}=\mathrm{X}$-phos, $\mathbf{L 5}=$ tricyclohexylphosphine, $\mathbf{L 6}=2$-(dicyclohexylphosphino)biphenyl, $\mathbf{L 7}=$ 1,3-bis(diphenylphosphino)propane, $\mathbf{L 8}=1,10$-phenanthroline, $\mathbf{L 9}=$ tri-tert-butylphosphine tetrafluoroborate.

\section{General procedure for the synthesis of 2,6-dibromophenylpyrroles}

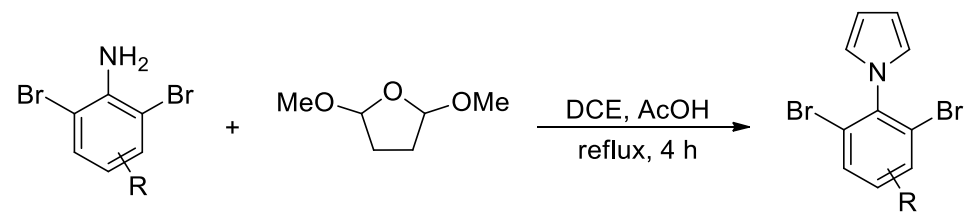

To a $100 \mathrm{~mL}$ round bottom flask was added 2,6-dibromoanilines (17.4 mmol, 1.0 equiv), dichloroethane $(20.0 \mathrm{~mL})$, acetic acid $(20.0 \mathrm{~mL})$, and 2,5-dimethoxytetrahydrofuran $(5.3 \mathrm{~mL})$. The reaction mixture was then heated to reflux for 4 hours. The mixture was diluted with $\mathrm{CH}_{2} \mathrm{Cl}_{2}$, and rinsed with $\mathrm{H}_{2} \mathrm{O}$. The organic layer was separated, washed with saturated aqueous $\mathrm{K}_{2} \mathrm{CO}_{3}$, dried with $\mathrm{MgSO}_{4}$, and concentrated. The residue was purified by column chromatography on silica gel to provide the desired product.

\section{General procedure for the Pd-catalyzed cyclization reaction of}

\section{2,6-dibromophenylpyrroles with alkynes}

A flame-dried Schlenk tube with a magnetic stir bar was charged with 2,6-dibromophenylpyrrole (0.25 mmol), alkyne (0.75 mmol), $\mathrm{K}_{2} \mathrm{CO}_{3}(103.7 \mathrm{mg}, 0.75$ mmol), Pd(TFA) 2 (16.6 mg, $50.0 \mu \mathrm{mol}), t \mathrm{Bu}_{3} \mathrm{P} \cdot \mathrm{HBF}_{4}(29.0 \mathrm{mg}, 0.10 \mathrm{mmol}), \mathrm{Y}(\mathrm{OTf})_{3}$ (268.1 $\mathrm{mg}, 0.50 \mathrm{mmol}$ ) and $\mathrm{MeCN}(2.0 \mathrm{~mL})$ under $\mathrm{N}_{2}$. The tube was sealed with a teflon-coated screw cap and the reaction solution was heated at $120{ }^{\circ} \mathrm{C}$ for $24 \mathrm{~h}$. The mixture was then cooled to ambient temperature, diluted with $10 \mathrm{~mL}$ of $\mathrm{CH}_{2} \mathrm{Cl}_{2}$, filtered through a celite pad, and washed with $10-20 \mathrm{~mL}$ of $\mathrm{CH}_{2} \mathrm{Cl}_{2}$. The combined organic phases were concentrated and the residue was purified by column chromatography on silica gel to provide the desired product. 


\section{Experimental data for the described substances}<smiles>Cc1cc(Br)c(-n2cccc2)c(Br)c1</smiles>

\section{1-(2,6-Dibromo-4-methylphenyl)-1H-pyrrole (1b)}

Purification by column chromatography on silica gel (dichloromethane/petroleum ether $=1 / 10, \mathrm{v} / \mathrm{v})$ afforded $\mathbf{1 b}$ as a white solid $\left(4.9 \mathrm{~g}, 90 \%\right.$ yield). M.p.: $88-90{ }^{\circ} \mathrm{C} .{ }^{1} \mathrm{H}$ NMR (400 MHz, $\left.\mathrm{CDCl}_{3}\right): \delta=2.38(\mathrm{~s}, 3 \mathrm{H}), 6.37(\mathrm{q}, J=2.0 \mathrm{~Hz}, 2 \mathrm{H}), 6.66(\mathrm{q}, J=2.0$ $\mathrm{Hz}, 2 \mathrm{H}), 7.46$ (s, 2H) ppm. ${ }^{13} \mathrm{C} \mathrm{NMR}\left(100 \mathrm{MHz}, \mathrm{CDCl}_{3}\right): \delta=20.7,109.2,121.8$, 123.7, 133.0, 137.2, 141.5 ppm. HRMS $\left(\mathrm{ESI}^{+}\right)$: calcd for $\mathrm{C}_{11} \mathrm{H}_{10} \mathrm{Br}_{2} \mathrm{~N}[\mathrm{M}+\mathrm{H}]^{+}$ 313.9180, found 313.9178.<smiles>CC(C)(C)c1cc(Br)c(-n2cccc2)c(Br)c1</smiles>

\section{1-(2,6-Dibromo-4-(tert-butyl)phenyl)-1H-pyrrole (1c)}

Purification by column chromatography on silica gel (dichloromethane/petroleum ether $=1 / 10, \mathrm{v} / \mathrm{v})$ afforded $1 \mathrm{c}$ as a white solid $(5.9 \mathrm{~g}, 95 \%$ yield $)$. M.p.: $70-72{ }^{\circ} \mathrm{C} .{ }^{1} \mathrm{H}$ NMR (400 MHz, $\left.\mathrm{CDCl}_{3}\right): \delta=1.36(\mathrm{~s}, 9 \mathrm{H}), 6.37(\mathrm{t}, J=2.0 \mathrm{~Hz}, 2 \mathrm{H}), 6.68(\mathrm{t}, J=2.0$ $\mathrm{Hz}, 2 \mathrm{H}), 7.63$ (s, 2H) ppm. ${ }^{13} \mathrm{C} \mathrm{NMR}\left(100 \mathrm{MHz}, \mathrm{CDCl}_{3}\right): \delta=31.2,35.1,109.2,121.8$, 123.7, 129.7, 137.1, 154.7 ppm. HRMS $\left(\mathrm{ESI}^{+}\right)$: calcd for $\mathrm{C}_{14} \mathrm{H}_{16} \mathrm{Br}_{2} \mathrm{~N}[\mathrm{M}+\mathrm{H}]^{+}$ 355.9649 , found 355.9654 .<smiles>Cc1cc(Br)c(-n2cccc2)c(Br)c1C</smiles> 


\section{1-(2,6-Dibromo-3,4-dimethylphenyl)-1H-pyrrole (1d)}

Purification by column chromatography on silica gel (dichloromethane/petroleum ether $=1 / 10, \mathrm{v} / \mathrm{v})$ afforded $1 \mathbf{d}$ as a white solid $\left(4.9 \mathrm{~g}, 86 \%\right.$ yield). M.p.: $76-78{ }^{\circ} \mathrm{C} .{ }^{1} \mathrm{H}$ NMR (400 MHz, $\mathrm{CDCl}_{3}$ ): $\delta=2.38$ (s, 3H), 2.40 (s, 3H), 6.36-6.37 (m, 2H), 6.64-6.65 $(\mathrm{m}, 2 \mathrm{H}), 7.45$ (s, 1H) ppm. ${ }^{13} \mathrm{C} \mathrm{NMR}\left(100 \mathrm{MHz}, \mathrm{CDCl}_{3}\right): \delta=20.5,21.2,109.1,120.3$, 121.7, 127.4, 132.7, 137.5, 137.7, 139.8 ppm. HRMS (ESI ${ }^{+}$): calcd for $\mathrm{C}_{12} \mathrm{H}_{12} \mathrm{Br}_{2} \mathrm{~N}$ $[\mathrm{M}+\mathrm{H}]^{+}$327.9336, found 327.9339.<smiles>Brc1cc(-c2ccccc2)cc(Br)c1-n1cccc1</smiles>

\section{1-(3,5-Dibromo-[1,1'-biphenyl]-4-yl)-1H-pyrrole (1e)}

Purification by column chromatography on silica gel (dichloromethane/petroleum ether $=1 / 6, \mathrm{v} / \mathrm{v})$ afforded $1 \mathrm{e}$ as a white solid $(5.6 \mathrm{~g}, 85 \%$ yield $)$. M.p.: $148-150{ }^{\circ} \mathrm{C} .{ }^{1} \mathrm{H}$ NMR (400 MHz, $\left.\mathrm{CDCl}_{3}\right): \delta=6.40-6.42(\mathrm{~m}, 2 \mathrm{H}), 6.73-6.74(\mathrm{~m}, 2 \mathrm{H}), 7.44-7.51(\mathrm{~m}$, 3H), 7.57-7.59 (m, 2H), 7.85 (s, 2H) ppm. $\left.{ }^{13} \mathrm{C} \mathrm{NMR} \mathrm{(100} \mathrm{MHz,} \mathrm{CDCl}_{3}\right): \delta=109.5$, $121.8,124.4,127.3,128.9,129.3,131.0,137.7,138.6,144.1$ ppm. HRMS $\left(\mathrm{ESI}^{+}\right)$: calcd for $\mathrm{C}_{16} \mathrm{H}_{12} \mathrm{Br}_{2} \mathrm{~N}[\mathrm{M}+\mathrm{H}]^{+}$375.9336, found 375.9331 .<smiles>COc1cc(Br)c(-n2cccc2)c(Br)c1</smiles>

\section{1-(2,6-Dibromo-4-methoxyphenyl)-1H-pyrrole (1f)}

Purification by column chromatography on silica gel (dichloromethane/petroleum ether $=1 / 4$, v/v) afforded $\mathbf{1 f}$ as a white solid (5.2 g, 90\% yield). M.p.: $147-149{ }^{\circ} \mathrm{C} .{ }^{1} \mathrm{H}$ NMR (400 MHz, $\left.\mathrm{CDCl}_{3}\right): \delta=3.85(\mathrm{~s}, 3 \mathrm{H}), 6.36(\mathrm{~d}, J=1.6 \mathrm{~Hz}, 2 \mathrm{H}), 6.65(\mathrm{~d}, J=1.6$ $\mathrm{Hz}, 2 \mathrm{H}), 7.18$ (s, 2H) ppm. ${ }^{13} \mathrm{C} \mathrm{NMR}\left(100 \mathrm{MHz}, \mathrm{CDCl}_{3}\right): \delta=56.2,109.2,118.0$, 122.1, 124.3, 132.9, 159.9 ppm. HRMS $\left(\mathrm{ESI}^{+}\right)$: calcd for $\mathrm{C}_{11} \mathrm{H}_{10} \mathrm{Br}_{2} \mathrm{NO}[\mathrm{M}+\mathrm{H}]^{+}$ 
329.9129, found 329.9128 .<smiles>FC(F)(F)c1cc(Br)c(-n2cccc2)c(Br)c1</smiles>

\section{1-(2,6-Dibromo-4-(trifluoromethyl)phenyl)-1H-pyrrole (1g)}

Purification by column chromatography on silica gel (dichloromethane/petroleum ether $=1 / 15, \mathrm{v} / \mathrm{v}$ ) afforded $1 \mathrm{~g}$ as a white solid (5.6 g, 88\% yield). M.p.: $108-110{ }^{\circ} \mathrm{C}$. ${ }^{1} \mathrm{H}$ NMR $\left(400 \mathrm{MHz}, \mathrm{CDCl}_{3}\right): \delta=6.41(\mathrm{t}, J=2.0 \mathrm{~Hz}, 2 \mathrm{H}), 6.68(\mathrm{t}, J=2.0 \mathrm{~Hz}, 2 \mathrm{H})$, $7.92(\mathrm{~s}, 2 \mathrm{H}) \mathrm{ppm} .{ }^{13} \mathrm{C} \mathrm{NMR}\left(100 \mathrm{MHz}, \mathrm{CDCl}_{3}\right): \delta=110.1,121.3,122.2$ (q, $J=272.0$ $\mathrm{Hz}), 124.9,129.6(\mathrm{q}, J=4.0 \mathrm{~Hz}), 132.8(\mathrm{q}, J=3.4 \mathrm{~Hz}), 143.1 \mathrm{ppm} . \mathrm{HRMS}\left(\mathrm{ESI}^{+}\right)$: calcd for $\mathrm{C}_{11} \mathrm{H}_{7} \mathrm{Br}_{2} \mathrm{~F}_{3} \mathrm{~N}[\mathrm{M}+\mathrm{H}]^{+}$367.8897, found 367.8893 .<smiles>Clc1cc(Br)c(-n2cccc2)c(Br)c1</smiles>

\section{1-(2,6-Dibromo-4-chlorophenyl)-1H-pyrrole (1h)}

Purification by column chromatography on silica gel (dichloromethane/petroleum ether $=1 / 10, \mathrm{v} / \mathrm{v})$ afforded $\mathbf{1 h}$ as a white solid (5.2 g, 89\% yield). M.p.: $100-102{ }^{\circ} \mathrm{C}$. ${ }^{1} \mathrm{H}$ NMR (400 MHz, $\left.\mathrm{CDCl}_{3}\right): \delta=6.38(\mathrm{~d}, J=2.0 \mathrm{~Hz}, 2 \mathrm{H}), 6.65(\mathrm{~d}, J=2.0 \mathrm{~Hz}, 2 \mathrm{H})$, 7.66-7.67 (m, 2H) ppm. $\left.{ }^{13} \mathrm{C} \mathrm{NMR} \mathrm{(100} \mathrm{MHz,} \mathrm{CDCl}_{3}\right): \delta=109.8,121.6,124.5,132.2$, 135.5, 138.8 ppm. HRMS $\left(\mathrm{ESI}^{+}\right)$: calcd for $\mathrm{C}_{10} \mathrm{H}_{7} \mathrm{Br}_{2} \mathrm{ClN}[\mathrm{M}+\mathrm{H}]^{+}$333.8634, found 333.8638 . 
<smiles>CCOC(=O)c1cc(Br)c(-n2cccc2)c(Br)c1</smiles>

\section{Ethyl 3,5-dibromo-4-(1H-pyrrol-1-yl)benzoate (1i)}

Purification by column chromatography on silica gel (dichloromethane/petroleum ether $=1 / 2, \mathrm{v} / \mathrm{v})$ afforded $\mathbf{1 i}$ as a white solid $(3.2 \mathrm{~g}, 50 \%$ yield $)$. M.p.: $130-132{ }^{\circ} \mathrm{C} .{ }^{1} \mathrm{H}$ NMR (400 MHz, $\left.\mathrm{CDCl}_{3}\right): \delta=1.43(\mathrm{t}, J=7.2 \mathrm{~Hz}, 3 \mathrm{H}), 4.43(\mathrm{q}, J=7.2 \mathrm{~Hz}, 2 \mathrm{H}), 6.40$ (t, $J=2.4 \mathrm{~Hz}, 2 \mathrm{H}), 6.68$ (t, $J=2.4 \mathrm{~Hz}, 2 \mathrm{H}), 8.29$ (s, 2H) ppm. ${ }^{13} \mathrm{C}$ NMR (100 MHz, $\left.\mathrm{CDCl}_{3}\right): \delta=14.4,62.3,109.9,121.3,124.2,132.7,133.5,143.4,163.6$ ppm. HRMS $\left(\mathrm{ESI}^{+}\right)$: calcd for $\mathrm{C}_{13} \mathrm{H}_{12} \mathrm{Br}_{2} \mathrm{NO}_{2}[\mathrm{M}+\mathrm{H}]^{+} 371.9235$, found 371.9236 .<smiles>N#Cc1cc(Br)c(-n2cccc2)c(Br)c1</smiles>

\section{3,5-Dibromo-4-(1H-pyrrol-1-yl)benzonitrile (1j)}

Purification by column chromatography on silica gel (dichloromethane/petroleum ether $=1 / 2, \mathrm{v} / \mathrm{v})$ afforded $1 \mathrm{i}$ as a white solid (4.2 g, $75 \%$ yield). M.p.: $168-170{ }^{\circ} \mathrm{C} .{ }^{1} \mathrm{H}$ NMR (400 MHz, $\left.\mathrm{CDCl}_{3}\right): \delta=6.42(\mathrm{t}, J=2.0 \mathrm{~Hz}, 2 \mathrm{H}), 6.67(\mathrm{t}, J=2.0 \mathrm{~Hz}, 2 \mathrm{H}), 7.95$ (s, 2H) ppm. $\left.{ }^{13} \mathrm{C} \mathrm{NMR} \mathrm{(100} \mathrm{MHz,} \mathrm{CDCl}_{3}\right): \delta=110.4,114.8,115.5,121.1,125.1$, 135.7, 144.2 ppm. HRMS (ESI ${ }^{+}$): calcd for $\mathrm{C}_{11} \mathrm{H}_{7} \mathrm{Br}_{2} \mathrm{~N}_{2}[\mathrm{M}+\mathrm{H}]^{+} 324.8976$, found 324.8972 .<smiles>c1ccc(-c2c(-c3ccccc3)c3ccc4c(-c5ccccc5)c(-c5ccccc5)c5cccc2c5n34)cc1</smiles>

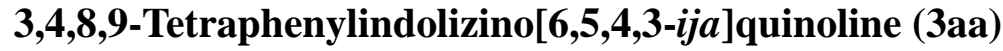

Purification by column chromatography on silica gel (dichloromethane/petroleum 
ether $=1 / 8, \mathrm{v} / \mathrm{v})$ afforded 3aa as a yellow solid (89 mg, $72 \%$ yield). M.p.: $>250{ }^{\circ} \mathrm{C}$. ${ }^{1} \mathrm{H}$ NMR (400 MHz, $\left.\mathrm{CDCl}_{3}\right): \delta=6.72(\mathrm{~s}, 2 \mathrm{H}), 7.22-7.26(\mathrm{~m}, 10 \mathrm{H}), 7.27-7.29(\mathrm{~m}, 5 \mathrm{H})$, 7.31-7.35 (m, 8H) ppm. ${ }^{13} \mathrm{C} \mathrm{NMR}\left(100 \mathrm{MHz}, \mathrm{CDCl}_{3}\right): \delta=106.8,119.5,123.4,126.4$, 127.1, 127.3, 128.0, 128.6, 130.1, 130.4, 131.0, 131.4, 132.6, 137.5, 137.8 ppm. HRMS (ESI ${ }^{+}$): calcd for $\mathrm{C}_{38} \mathrm{H}_{26} \mathrm{~N}[\mathrm{M}+\mathrm{H}]^{+}$496.2065, found 496.2063.<smiles>Cc1ccc(-c2c(-c3ccc(C)cc3)c3ccc4c(-c5ccc(C)cc5)c(-c5ccc(C)cc5)c5cccc2c5n34)cc1</smiles>

\section{3,4,8,9-Tetra-p-tolylindolizino[6,5,4,3-ija]quinoline (3ab)}

Purification by column chromatography on silica gel (dichloromethane/petroleum ether $=1 / 8, \mathrm{v} / \mathrm{v})$ afforded 3ab as a yellow solid (90 mg, 65\% yield). M.p.: $>250{ }^{\circ} \mathrm{C}$. ${ }^{1} \mathrm{H} \mathrm{NMR}\left(400 \mathrm{MHz}, \mathrm{CDCl}_{3}\right): \delta=2.33(\mathrm{~s}, 6 \mathrm{H}), 2.35$ (s, 6H), $6.70(\mathrm{~s}, 2 \mathrm{H}), 7.07(\mathrm{~d}, J=$ $8.0 \mathrm{~Hz}, 4 \mathrm{H}), 7.13(\mathrm{bs}, 8 \mathrm{H}), 7.20-7.23(\mathrm{~m}, 7 \mathrm{H}) \mathrm{ppm} .{ }^{13} \mathrm{C} \mathrm{NMR}\left(100 \mathrm{MHz}, \mathrm{CDCl}_{3}\right): \delta=$ $21.45,21.48,106.6,119.3,123.2,126.6,128.2$, 128.7, 129.3, 129.8, 130.3, 130.9, 131.2, 132.5, 134.7, 135.0, 136.5, 136.7 ppm. HRMS $\left(\mathrm{ESI}^{+}\right)$: calcd for $\mathrm{C}_{42} \mathrm{H}_{34} \mathrm{~N}$ $[\mathrm{M}+\mathrm{H}]^{+}$552.2691, found 552.2695.<smiles></smiles>

\section{3,4,8,9-Tetrakis(4-(tert-butyl)phenyl)indolizino[6,5,4,3-ija]quinoline (3ac)}

Purification by column chromatography on silica gel (dichloromethane/petroleum ether $=1 / 8, \mathrm{v} / \mathrm{v})$ afforded 3ac as a yellow solid (121 mg, 67\% yield). M.p.: $>250{ }^{\circ} \mathrm{C}$. ${ }^{1} \mathrm{H}$ NMR (400 MHz, $\left.\mathrm{CDCl}_{3}\right): \delta=1.28$ (s, 18H), 1.30 (s, 18H), 6.79 (s, 2H), 7.13 (d, $J$ $=8.0 \mathrm{~Hz}, 4 \mathrm{H}), 7.22(\mathrm{bs}, 8 \mathrm{H}), 7.28-7.30(\mathrm{~m}, 7 \mathrm{H}) \mathrm{ppm} .{ }^{13} \mathrm{C} \mathrm{NMR}\left(100 \mathrm{MHz}, \mathrm{CDCl}_{3}\right): \delta$ $=31.4,31.5,34.61,34.63,106.6,119.2,123.2,124.6,125.2,126.5,128.1,130.0$, 130.7, 131.4, 134.7, 135.0, 149.7, 149.9 ppm. HRMS $\left(\mathrm{ESI}^{+}\right)$: calcd for $\mathrm{C}_{54} \mathrm{H}_{58} \mathrm{~N}$ 
<smiles>Cc1cccc(-c2c(-c3cccc(C)c3)c3ccc4c(-c5cccc(C)c5)c(-c5cccc(C)c5)c5cccc2c5n34)c1</smiles>

\section{3,4,8,9-Tetra-m-tolylindolizino[6,5,4,3-ija]quinoline (3ad)}

Purification by column chromatography on silica gel (dichloromethane/petroleum ether $=1 / 8, \mathrm{v} / \mathrm{v})$ afforded 3ad as a yellow solid $(83 \mathrm{mg}, 60 \%$ yield $)$. M.p.: $>250{ }^{\circ} \mathrm{C}$. ${ }^{1} \mathrm{H}$ NMR $\left(400 \mathrm{MHz}, \mathrm{CDCl}_{3}\right): \delta=2.28(\mathrm{~s}, 6 \mathrm{H}), 2.31(\mathrm{~s}, 6 \mathrm{H}), 6.73(\mathrm{~s}, 2 \mathrm{H}), 7.05-7.07(\mathrm{~m}$, 8H), 7.11-7.24 (m, 11H) ppm. ${ }^{13} \mathrm{C}$ NMR (100 MHz, $\left.\mathrm{CDCl}_{3}\right): \delta=21.5,21.6,106.6$, $119.4,123.3,126.5,127.5,127.77,127.80,128.0,128.05,128.07,128.4,130.1,131.1$, 131.3, 131.6, 132.5, 137.4, 137.5, 137.8, 138.0 ppm. HRMS $\left(\mathrm{ESI}^{+}\right)$: calcd for $\mathrm{C}_{42} \mathrm{H}_{34} \mathrm{~N}[\mathrm{M}+\mathrm{H}]^{+}$552.2691, found 552.2692.<smiles></smiles>

\section{3,4,8,9-Tetrakis(4-(trifluoromethyl)phenyl)indolizino $[6,5,4,3-i j a] q u i n o l i n e ~(3 a e)$}

Purification by column chromatography on silica gel (dichloromethane/petroleum ether $=1 / 10$, v/v) afforded 3ae as a yellow solid (106 mg, 55\% yield). M.p.: $>250{ }^{\circ} \mathrm{C}$. ${ }^{1} \mathrm{H}$ NMR $\left(400 \mathrm{MHz}, \mathrm{CDCl}_{3}\right): \delta=6.71(\mathrm{~s}, 2 \mathrm{H}), 7.19(\mathrm{~d}, J=8.0 \mathrm{~Hz}, 2 \mathrm{H}), 7.32(\mathrm{~d}, J=$ $7.6 \mathrm{~Hz}, 1 \mathrm{H}), 7.35-7.38(\mathrm{~m}, 4 \mathrm{H}), 7.44(\mathrm{~d}, J=8.0 \mathrm{~Hz}, 4 \mathrm{H}), 7.57(\mathrm{~d}, J=8.0 \mathrm{~Hz}, 4 \mathrm{H})$, $7.63(\mathrm{~d}, J=8.0 \mathrm{~Hz}, 4 \mathrm{H}) \mathrm{ppm} .{ }^{13} \mathrm{C} \mathrm{NMR}\left(100 \mathrm{MHz}, \mathrm{CDCl}_{3}\right): \delta=107.5,120.0,124.0$, $124.1(\mathrm{q}, J=264.0 \mathrm{~Hz}), 124.2(\mathrm{q}, J=270.0 \mathrm{~Hz}), 125.5(\mathrm{q}, J=4.0 \mathrm{~Hz}), 125.8,126.0(\mathrm{q}$, $J=4.0 \mathrm{~Hz}), 127.6,129.3,129.9(\mathrm{q}, J=32.0 \mathrm{~Hz}), 130.1(\mathrm{q}, J=32.0 \mathrm{~Hz}), 130.4,130.6$, 131.3, 132.9, 140.5, 141.0 ppm. ${ }^{19} \mathrm{~F}$ NMR $\left(376 \mathrm{MHz} \mathrm{CDCl}_{3}\right): \delta=-62.59,-62.55 \mathrm{ppm}$. HRMS (ESI ${ }^{+}$): calcd for $\mathrm{C}_{42} \mathrm{H}_{22} \mathrm{~F}_{12} \mathrm{~N}[\mathrm{M}+\mathrm{H}]^{+}$768.1561, found 768.1570. 
<smiles></smiles>

\section{3,4,8,9-Tetrakis(4-fluorophenyl)indolizino[6,5,4,3-ija]quinoline (3af)}

Purification by column chromatography on silica gel (dichloromethane/petroleum ether $=1 / 10, \mathrm{v} / \mathrm{v})$ afforded 3af as a yellow solid (106 mg, 75\% yield). M.p.: $>250{ }^{\circ} \mathrm{C}$. ${ }^{1} \mathrm{H}$ NMR $\left(400 \mathrm{MHz}, \mathrm{CDCl}_{3}\right): \delta=6.61(\mathrm{t}, J=8.8 \mathrm{~Hz}, 2 \mathrm{H}), 6.70-6.75(\mathrm{~m}, 4 \mathrm{H})$, 6.96-7.06 (m, 7H), 7.17-7.21 (m, 5H), 7.25-7.26 (m, 1H), 7.27-7.29 (m, 2H) ppm. ${ }^{13} \mathrm{C}$ NMR (100 MHz, $\left.\mathrm{CDCl}_{3}\right): \delta=106.9,115.3(\mathrm{~d}, J=21.0 \mathrm{~Hz}), 115.9(\mathrm{~d}, J=22.0 \mathrm{~Hz})$, $119.5,123.6,126.3,128.0,129.4,130.7,131.9(\mathrm{~d}, J=8.0 \mathrm{~Hz}), 132.6(\mathrm{~d}, J=8.0 \mathrm{~Hz})$, $133.1(\mathrm{~d}, J=4.0 \mathrm{~Hz}), 133.4(\mathrm{~d}, J=3.0 \mathrm{~Hz}), 162.0(\mathrm{~d}, J=245.0 \mathrm{~Hz}), 162.1(\mathrm{~d}, J=$ $245.0 \mathrm{~Hz}$ ) ppm. ${ }^{19} \mathrm{~F}$ NMR $\left(376 \mathrm{MHz}, \mathrm{CDCl}_{3}\right): \delta=-114.56,-114.26 \mathrm{ppm}$. HRMS $\left(\mathrm{ESI}^{+}\right.$): calcd for $\mathrm{C}_{38} \mathrm{H}_{22} \mathrm{~F}_{4} \mathrm{~N}[\mathrm{M}+\mathrm{H}]^{+}$568.1688, found 568.1686.<smiles></smiles>

\section{3,4,8,9-Tetrakis(4-chlorophenyl)indolizino[6,5,4,3-ija $]$ quinoline (3ag)}

Purification by column chromatography on silica gel (dichloromethane/petroleum ether $=1 / 8, \mathrm{v} / \mathrm{v})$ afforded 3ag as a yellow solid (100 mg, 63\% yield). M.p.: $>250{ }^{\circ} \mathrm{C}$. ${ }^{1} \mathrm{H}$ NMR $\left(400 \mathrm{MHz}, \mathrm{CDCl}_{3}\right): \delta=6.69$ (s, 2H), 7.14-7.23 (m, 11H), 7.26-7.33 (m, 8H) ppm. ${ }^{13} \mathrm{C}$ NMR $\left(100 \mathrm{MHz}, \mathrm{CDCl}_{3}\right): \delta=107.1,119.6,123.7,126.0,127.8,128.7$, $129.1,129.2,130.5,131.6,132.3,132.7,133.4,133.6,135.5,135.8$ ppm. HRMS $\left(\mathrm{ESI}^{+}\right.$): calcd for $\mathrm{C}_{38} \mathrm{H}_{22} \mathrm{Cl}_{4} \mathrm{~N}[\mathrm{M}+\mathrm{H}]^{+}$632.0506, found 632.0506 . 
<smiles>N#Cc1cccc(-c2c(-c3cccc(C#N)c3)c3ccc4c(-c5cccc(C#N)c5)c(-c5cccc(C#N)c5)c5cccc2c5n34)c1</smiles>

\section{3,3',3",3'"'-(Indolizino[6,5,4,3-ija ]quinoline-3,4,8,9-tetrayl)tetrabenzonitrile (3ah)}

Purification by column chromatography on silica gel (ethyl acetate/petroleum ether = 1/3, v/v) afforded 3ah as a yellow solid (60 mg, $40 \%$ yield). M.p.: > $250{ }^{\circ} \mathrm{C} .{ }^{1} \mathrm{H}$ NMR $\left(400 \mathrm{MHz}, \mathrm{CDCl}_{3}\right): \delta=6.69(\mathrm{~s}, 2 \mathrm{H}), 7.15(\mathrm{~d}, J=8.0 \mathrm{~Hz}, 2 \mathrm{H}), 7.37(\mathrm{t}, J=8.0 \mathrm{~Hz}, 1 \mathrm{H})$, 7.44-7.52 (m, 10H), 7.60-7.65 (m, 6H) ppm. $\left.{ }^{13} \mathrm{C} \mathrm{NMR} \mathrm{(100} \mathrm{MHz,} \mathrm{CDCl}_{3}\right): \delta=107.7$, $113.0,113.5,118.4,120.1,124.3,125.5,127.5,128.7,129.6,130.0,130.1,131.7$, 131.8, 132.9, 133.5, 134.2, 134.6, 135.46, 135.50, 137.7, 138.1 ppm. HRMS (ESI $\left.{ }^{+}\right)$: calcd for $\mathrm{C}_{42} \mathrm{H}_{22} \mathrm{~N}_{5}[\mathrm{M}+\mathrm{H}]^{+}$596.1875, found 596.1877.<smiles>O=[W]Oc1ccc(-c2c(-c3ccc(O)cc3)c3cccc4c(-c5ccc(OO)cc5)c(-c5ccc(O)cc5)c5ccc2n5c43)cc1</smiles>

\section{3,4,8,9-Tetrakis(4-(hexyloxy)phenyl)indolizino[6,5,4,3-ija]quinoline (3ai)}

Purification by column chromatography on silica gel (dichloromethane/petroleum ether $=1 / 2, \mathrm{v} / \mathrm{v})$ afforded 3ai as a yellow solid (78 mg, 35\% yield). M.p.: $>250{ }^{\circ} \mathrm{C}$. ${ }^{1} \mathrm{H}$ NMR (400 MHz, $\left.\mathrm{CDCl}_{3}\right): \delta=0.90-0.94$ (m, 12H), 1.33-1.38 (m, 16H), 1.43-1.50 $(\mathrm{m}, 8 \mathrm{H}), 1.75-1.83(\mathrm{~m}, 8 \mathrm{H}), 3.95(\mathrm{q}, J=6.8 \mathrm{~Hz}, 8 \mathrm{H}), 6.72(\mathrm{~s}, 2 \mathrm{H}), 6.80(\mathrm{~d}, J=8.8 \mathrm{~Hz}$, $4 \mathrm{H}), 6.86(\mathrm{~d}, J=8.4 \mathrm{~Hz}, 4 \mathrm{H}), 7.13(\mathrm{~d}, J=8.4 \mathrm{~Hz}, 4 \mathrm{H}), 7.22-7.24(\mathrm{~m}, 7 \mathrm{H}) \mathrm{ppm} .{ }^{13} \mathrm{C}$ NMR (100 MHz, $\left.\mathrm{CDCl}_{3}\right): \delta=14.2,22.8,25.9,29.5,31.8,68.03,68.07,106.5,114.0$, $114.6,119.2,123.2,126.8,128.3,129.6,129.9,130.1,131.0,131.5,132.1,132.4$, 158.1, 158.2 ppm. HRMS $\left(\mathrm{ESI}^{+}\right)$: calcd for $\mathrm{C}_{62} \mathrm{H}_{74} \mathrm{NO}_{4}[\mathrm{M}+\mathrm{H}]^{+}$896.5618, found 896.5612 . 
<smiles></smiles>

\section{Tetramethyl}

3,3',3' ,3'"'-(Indolizino[6,5,4,3-ija $]$ quinoline-3,4,8,9-tetrayl)tetrabenzoate (3aj)

Purification by column chromatography on silica gel (dichloromethane) afforded 3aj as a yellow solid (49 mg, 27\% yield). M.p.: > $250{ }^{\circ} \mathrm{C} .{ }^{1} \mathrm{H}$ NMR $\left(400 \mathrm{MHz}, \mathrm{CDCl}_{3}\right): \delta$ $=3.89(\mathrm{~s}, 12 \mathrm{H}), 6.70(\mathrm{~s}, 2 \mathrm{H}), 7.16(\mathrm{~d}, J=7.6 \mathrm{~Hz}, 2 \mathrm{H}), 7.27-7.45(\mathrm{~m}, 9 \mathrm{H}), 7.92-7.94$ $(\mathrm{m}, 4 \mathrm{H}), 8.01(\mathrm{~d}, J=5.6 \mathrm{~Hz}, 2 \mathrm{H}), 8.09(\mathrm{~s}, 2 \mathrm{H}) \mathrm{ppm} .{ }^{13} \mathrm{C} \mathrm{NMR}\left(100 \mathrm{MHz}, \mathrm{CDCl}_{3}\right): \delta=$ $52.30,52.32,107.2,119.7,123.8,126.0,127.9,128.5,128.7,128.9,129.1,129.5$, $130.3,130.75,130.82,131.4,132.2,132.7,134.9,135.7,137.3,137.7,166.9,167.0$ ppm. HRMS (ESI ${ }^{+}$: calcd for $\mathrm{C}_{46} \mathrm{H}_{33} \mathrm{NNaO}_{8}[\mathrm{M}+\mathrm{Na}]^{+}$750.2104, found 750.2105.<smiles>CC(=O)c1cccc(-c2c(-c3cccc(C(C)=O)c3)c3ccc4c(-c5cccc(C(C)=O)c5)c(-c5cccc(C(C)=O)c5)c5cccc2c5n34)c1</smiles>

\section{1,1',1",1"'-(Indolizino[6,5,4,3-ija]quinoline-3,4,8,9-tetrayltetrakis(benzene-3,1-di yl))tetraethanone (3ak)}

Purification by column chromatography on silica gel (ethyl acetate/petroleum ether $=$ $1 / 2$, v/v) afforded 3ak as a yellow solid (80 mg, $48 \%$ yield). M.p.: > $250{ }^{\circ} \mathrm{C} .{ }^{1} \mathrm{H}$ NMR $\left(400 \mathrm{MHz}, \mathrm{CDCl}_{3}\right): \delta=2.49(\mathrm{~s}, 6 \mathrm{H}), 2.52(\mathrm{~s}, 6 \mathrm{H}), 6.72(\mathrm{~s}, 2 \mathrm{H}), 7.20(\mathrm{~d}, J=8.0 \mathrm{~Hz}$, 2H), $7.31(\mathrm{t}, J=8.0 \mathrm{~Hz}, 1 \mathrm{H}), 7.40(\mathrm{t}, J=8.0 \mathrm{~Hz}, 2 \mathrm{H}), 7.48(\mathrm{~m}, 4 \mathrm{H}), 7.56(\mathrm{~d}, J=7.2$ $\mathrm{Hz}, 2 \mathrm{H}), 7.84-7.93(\mathrm{~m}, 8 \mathrm{H}) \mathrm{ppm} .{ }^{13} \mathrm{C} \mathrm{NMR}\left(100 \mathrm{MHz}, \mathrm{CDCl}_{3}\right): \delta=26.7,26.8,107.3$, $110.2,119.8,123.9,126.0,127.6,127.8,128.7,129.3,129.6,130.5,130.9,131.1$, 
132.8, 134.9, 135.7, 137.1, 137.4, 137.7, 137.9, 197.7, 197.8 ppm. HRMS (ESI $\left.{ }^{+}\right)$: calcd for $\mathrm{C}_{46} \mathrm{H}_{33} \mathrm{NNaO}_{4}[\mathrm{M}+\mathrm{Na}]^{+}$686.2307, found 686.2308.<smiles>Cc1cc2c(-c3ccccc3)c(-c3ccccc3)c3cc(-c4ccccc4)c(-c4ccccc4)c-3ccc-2n1</smiles>

\section{6-Methyl-3,4,8,9-tetraphenylindolizino[6,5,4,3-ija $]$ quinoline (3ba)}

Purification by column chromatography on silica gel (dichloromethane/petroleum ether $=1 / 8, \mathrm{v} / \mathrm{v})$ afforded 3 ba as a yellow solid (64 mg, $50 \%$ yield). M.p.: $>250{ }^{\circ} \mathrm{C}$. ${ }^{1} \mathrm{H}$ NMR (400 MHz, $\left.\mathrm{CDCl}_{3}\right): \delta=2.32$ (s, 3H), 6.68 (s, 2H), 7.02 (s, 2H), 7.24-7.26 (m, $10 \mathrm{H}), 7.28-7.35(\mathrm{~m}, 10 \mathrm{H}) \mathrm{ppm} .{ }^{13} \mathrm{C} \mathrm{NMR}\left(100 \mathrm{MHz}, \mathrm{CDCl}_{3}\right): \delta=22.3,106.6,120.0$, 126.3, 127.1, 127.2, 127.9, 128.0, 128.6, 129.9, 130.4, 131.1, 131.4, 133.0, 137.6, 137.9 ppm. HRMS (ESI $\left.{ }^{+}\right)$: calcd for $\mathrm{C}_{39} \mathrm{H}_{27} \mathrm{NNa}[\mathrm{M}+\mathrm{Na}]^{+}$532.2041, found 532.2047.<smiles></smiles>

\section{6-(tert-Butyl)-3,4,8,9-tetraphenylindolizino[6,5,4,3-ija]quinoline (3ca)}

Purification by column chromatography on silica gel (dichloromethane/petroleum ether $=1 / 8, \mathrm{v} / \mathrm{v})$ afforded 3ca as a yellow solid $(62 \mathrm{mg}, 45 \%$ yield $)$. M.p.: $>250{ }^{\circ} \mathrm{C}$. ${ }^{1} \mathrm{H}$ NMR (400 MHz, $\mathrm{CDCl}_{3}$ ): $\delta=1.17$ (s, 9H), 6.67 (s, 2H), 7.22-7.35 (m, 22H) ppm. ${ }^{13} \mathrm{C} \mathrm{NMR}\left(100 \mathrm{MHz}, \mathrm{CDCl}_{3}\right): \delta=31.7,35.1,106.4,116.7,125.9,127.1,127.2,127.8$, 128.0, 128.5, 130.2, 130.4, 130.95, 131.00, 131.2, 137.7, 137.9, 146.2 ppm. HRMS $\left(\mathrm{ESI}^{+}\right)$: calcd for $\mathrm{C}_{42} \mathrm{H}_{33} \mathrm{NNa}[\mathrm{M}+\mathrm{Na}]^{+}$574.2511, found 574.2507. 
<smiles>Cc1=c(C)-c2c(-c3ccccc3)c(-c3ccccc3)c3c(c(-c4ccccc4)c(-c4ccccc4)c4ccc2n43)=c-1</smiles>

\section{5,6-Dimethyl-3,4,8,9-tetraphenylindolizino[6,5,4,3-ija]quinoline (3da)}

Purification by column chromatography on silica gel (dichloromethane/petroleum ether $=1 / 8, \mathrm{v} / \mathrm{v})$ afforded 3da as a yellow solid (55 mg, 42\% yield). M.p.: $>250{ }^{\circ} \mathrm{C}$. ${ }^{1} \mathrm{H}$ NMR (400 MHz, $\left.\mathrm{CDCl}_{3}\right): \delta=1.76$ (s, 3H), 2.29 (s, 3H), $6.45(\mathrm{~d}, J=4.0 \mathrm{~Hz}, 1 \mathrm{H})$, $6.62(\mathrm{~d}, J=4.0 \mathrm{~Hz}, 1 \mathrm{H}), 7.11-7.17(\mathrm{~m}, 8 \mathrm{H}), 7.20-7.25(\mathrm{~m}, 8 \mathrm{H}), 7.28-7.35(\mathrm{~m}, 5 \mathrm{H})$ ppm. ${ }^{13} \mathrm{C}$ NMR $\left(100 \mathrm{MHz}, \mathrm{CDCl}_{3}\right): \delta=19.4,22.2,106.2$. 106.4, 121.6, 124.19, 124.23, 126.3, 126.8, 127.0, 127.1, 127.5, 127.8, 127.9, 128.0, 128.4, 128.6, 129.8, $130.0,130.3,130.4,131.1,131.2,132.7,133.3,133.6,137.6,138.03,138.04,141.7$ ppm. HRMS $\left(\mathrm{ESI}^{+}\right)$: calcd for $\mathrm{C}_{40} \mathrm{H}_{29} \mathrm{NNa}[\mathrm{M}+\mathrm{Na}]^{+}$546.2198, found 546.2203.<smiles></smiles>

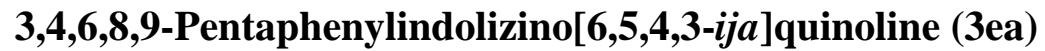

Purification by column chromatography on silica gel (dichloromethane/petroleum ether $=1 / 4, \mathrm{v} / \mathrm{v})$ afforded 3ea as a yellow solid (114 mg, 80\% yield). M.p.: $>250{ }^{\circ} \mathrm{C}$. ${ }^{1} \mathrm{H}$ NMR (400 MHz, $\left.\mathrm{CDCl}_{3}\right): \delta=6.74(\mathrm{~s}, 2 \mathrm{H}), 6.84(\mathrm{~s}, 2 \mathrm{H}), 7.24-7.26(\mathrm{~m}, 2 \mathrm{H})$, 7.28-7.35 (m, 19H), 7.40-7.42 (m, 2H), $7.46(\mathrm{~s}, 2 \mathrm{H}) \mathrm{ppm} .{ }^{13} \mathrm{C}$ NMR (100 MHz, $\left.\mathrm{CDCl}_{3}\right): \delta=107.1,118.4,126.71,126.74,127.0,127.2,127.3,127.8,128.1,128.7$, $128.8,130.2$, 130.4, 131.0, 131.6, 131.8, 136.6, 137.5, 137.7, 142.1 ppm. HRMS $\left(\mathrm{ESI}^{+}\right)$: calcd for $\mathrm{C}_{44} \mathrm{H}_{30} \mathrm{~N}[\mathrm{M}+\mathrm{H}]^{+}$572.2378, found 572.2369. 
<smiles></smiles>

\section{6-Methoxy-3,4,8,9-tetraphenylindolizino[6,5,4,3-ija]quinoline (3fa)}

Purification by column chromatography on silica gel (dichloromethane/petroleum ether $=1 / 2, \mathrm{v} / \mathrm{v})$ afforded $3 \mathrm{fa}$ as a yellow solid $\left(85 \mathrm{mg}, 65 \%\right.$ yield). M.p.: $>250{ }^{\circ} \mathrm{C}$. ${ }^{1} \mathrm{H}$ NMR (400 MHz, $\left.\mathrm{CDCl}_{3}\right): \delta=3.64$ (s, 3H), 6.70 (s, 2H), 6.79 (s, 2H), 7.23-7.25 (m, $10 \mathrm{H}), 7.26-7.32(\mathrm{~m}, 10 \mathrm{H}) \mathrm{ppm} .{ }^{13} \mathrm{C} \mathrm{NMR}\left(100 \mathrm{MHz}, \mathrm{CDCl}_{3}\right): \delta=55.6,104.9,106.8$, $127.2,127.3,127.6,127.7,128.0,128.3,128.7,129.7,130.4,130.9,131.9,137.5$, 137.8, 155.9 ppm. HRMS (ESI ${ }^{+}$: calcd for $\mathrm{C}_{39} \mathrm{H}_{27} \mathrm{NNaO}[\mathrm{M}+\mathrm{Na}]^{+}$548.1990, found 548.1982.<smiles>FC(F)(F)C1=Cc2c3ccc(c2-c2ccccc2)N(c2ccccc2)C(=C1)C(c1ccccc1)=C3c1ccccc1</smiles>

\section{3,4,8,9-Tetraphenyl-6-(trifluoromethyl)indolizino[6,5,4,3-ija]quinoline (3ga)}

Purification by column chromatography on silica gel (dichloromethane/petroleum ether $=1 / 8, \mathrm{v} / \mathrm{v})$ afforded 3ga as a yellow solid (56 mg, $40 \%$ yield). M.p.: $>250{ }^{\circ} \mathrm{C}$. ${ }^{1} \mathrm{H}$ NMR (400 MHz, $\left.\mathrm{CDCl}_{3}\right): \delta=6.84(\mathrm{~s}, 2 \mathrm{H}), 7.23-7.25(\mathrm{~m}, 5 \mathrm{H}), 7.26-7.37(\mathrm{~m}, 15 \mathrm{H})$, $7.48(\mathrm{~s}, 2 \mathrm{H}) \mathrm{ppm} .{ }^{13} \mathrm{C} \mathrm{NMR}\left(100 \mathrm{MHz}, \mathrm{CDCl}_{3}\right): \delta=108.1,115.7$ (q, $\left.J=4.0 \mathrm{~Hz}\right)$, $122.1(\mathrm{q}, J=262.0 \mathrm{~Hz}), 125.7(\mathrm{q}, J=32.0 \mathrm{~Hz}), 126.6,127.6,128.2,128.4,128.9$, 130.0, 130.3, 130.9, 132.6, 133.6, 136.96, 137.03 ppm. ${ }^{19} \mathrm{~F}$ NMR (376 MHz, $\left.\mathrm{CDCl}_{3}\right)$ : $\delta=-60.94$ ppm. HRMS $\left(\mathrm{ESI}^{+}\right)$: calcd for $\mathrm{C}_{39} \mathrm{H}_{24} \mathrm{~F}_{3} \mathrm{NNa}[\mathrm{M}+\mathrm{Na}]^{+}$586.1759, found 586.1761 . 
<smiles></smiles>

\section{6-Chloro-3,4,8,9-tetraphenylindolizino[6,5,4,3-ija]quinoline (3ha)}

Purification by column chromatography on silica gel (dichloromethane/petroleum ether $=1 / 8, \mathrm{v} / \mathrm{v})$ afforded 3ha as a yellow solid (40 mg, 30\% yield). M.p.: $>250{ }^{\circ} \mathrm{C}$. ${ }^{1} \mathrm{H}$ NMR (400 MHz, $\left.\mathrm{CDCl}_{3}\right): \delta=6.79(\mathrm{~s}, 2 \mathrm{H}), 7.18(\mathrm{~s}, 2 \mathrm{H}), 7.22-7.26(\mathrm{~m}, 7 \mathrm{H})$, 7.28-7.36 (m, 13H) ppm. ${ }^{13} \mathrm{C}$ NMR (100 MHz, $\left.\mathrm{CDCl}_{3}\right): \delta=107.7,118.6,127.46$, 127.52, 127.9, 128.0, 128.1, 128.8, 129.4, 129.7, 130.3, 130.9, 132.4, 137.1, 137.2 ppm. HRMS (ESI ${ }^{+}$): calcd for $\mathrm{C}_{38} \mathrm{H}_{25} \mathrm{ClN}[\mathrm{M}+\mathrm{H}]^{+}$530.1676, found 530.1671.<smiles></smiles>

Ethyl 3,4,8,9-tetraphenylindolizino[6,5,4,3-ija]quinoline-6-carboxylate (3ia)

Purification by column chromatography on silica gel (dichloromethane/petroleum ether $=1 / 1, \mathrm{v} / \mathrm{v})$ afforded 3ia as a yellow solid (85 mg, 35\% yield). M.p.: $>250{ }^{\circ} \mathrm{C}$. ${ }^{1} \mathrm{H}$ NMR (400 MHz, $\left.\mathrm{CDCl}_{3}\right): \delta=1.26(\mathrm{t}, J=7.2 \mathrm{~Hz}, 3 \mathrm{H}), 4.25(\mathrm{q}, J=7.2 \mathrm{~Hz}, 2 \mathrm{H})$, $6.79(\mathrm{~s}, 2 \mathrm{H}), 7.24-7.36(\mathrm{~m}, 20 \mathrm{H}), 7.97(\mathrm{~s}, 2 \mathrm{H}) \mathrm{ppm} .{ }^{13} \mathrm{C} \mathrm{NMR}\left(100 \mathrm{MHz}, \mathrm{CDCl}_{3}\right): \delta=$ 14.3, 61.1, 107.7, 120.7, 125.5, 126.2, 127.4, 127.5, 128.1, 128.4, 128.7, 130.3, 131.0, 132.0, 134.6, 137.18, 137.23, 167.2 ppm. HRMS (ESI ${ }^{+}$): calcd for $\mathrm{C}_{41} \mathrm{H}_{29} \mathrm{NNaO}_{2}$ $[\mathrm{M}+\mathrm{Na}]^{+}$590.2096, found 590.2098.<smiles></smiles>

3,4,8,9-Tetraphenylindolizino $[6,5,4,3-i j a] q u i n o l i n e-6-c a r b o n i t r i l e ~(3 j a)$ 
Purification by column chromatography on silica gel (dichloromethane/petroleum ether $=1 / 2, \mathrm{v} / \mathrm{v})$ afforded $3 \mathbf{j a}$ as a yellow solid (70 mg, 54\% yield). M.p.: $>250{ }^{\circ} \mathrm{C}$. ${ }^{1} \mathrm{H}$ NMR (400 MHz, $\mathrm{CDCl}_{3}$ ): $\delta=6.89$ (s, 2H), $7.22(\mathrm{~d}, J=7.2 \mathrm{~Hz}, 4 \mathrm{H}), 7.28-7.39$ (m, 16H), 7.49 (s, 2H) ppm. ${ }^{13} \mathrm{C}$ NMR (100 MHz, $\left.\mathrm{CDCl}_{3}\right): \delta=106.9,108.7,120.1,122.4$, 127.0, 127.77, 127.78, 128.2, 128.4, 129.0, 129.4, 130.2, 130.8, 132.9, 136.6, 136.7 ppm. HRMS (ESI ${ }^{+}$): calcd for $\mathrm{C}_{39} \mathrm{H}_{24} \mathrm{~N}_{2} \mathrm{Na}[\mathrm{M}+\mathrm{Na}]^{+}$543.1837, found 543.1841.

\section{Synthesis of ullazine-based sensitizers}<smiles>[R]c1ccc(-c2c(-c3ccc([R])cc3)c3ccc4c(-c5ccc([R])cc5)c(-c5ccc([R])cc5)c5cccc2c5n34)cc1</smiles>

3aa, $\mathrm{R}=\mathrm{H}$

3ai, $\mathrm{R}=\mathrm{OHexyl}$<smiles></smiles>

5c $(80 \%), \mathrm{R}=\mathrm{OHexyl}$

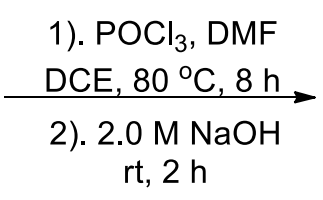<smiles>[R]c1ccc(-c2c(-c3ccc([R])cc3)c3ccc4c(-c5ccc([R])cc5)c(-c5ccc([R])cc5)c5c(C=O)ccc2c5n34)cc1</smiles>

4a $(78 \%), \mathrm{R}=\mathrm{H}$

4b $(72 \%), \mathrm{R}=$ OHexyl<smiles>[R]c1ccc(-c2c(-c3ccc([R])cc3)c3ccc4c(-c5ccc([R])cc5)c(-c5ccc([R])cc5)c5c(C=C(C#N)C(=O)O)ccc2c5n34)cc1</smiles>

$5 a(90 \%), \mathrm{R}=\mathrm{H}$ 5b $(85 \%), \mathrm{R}=\mathrm{OHexyl}$

\section{3,4,8,9-Tetraphenylindolizino[6,5,4,3-ija]quinoline-5-carbaldehyde (4a)}

To a Schlenk tube with a magnetic stir bar was added 3,4,8,9-tetraphenylindolizino[6,5,4,3-ija]quinoline 3aa (495 mg, $1.0 \mathrm{mmol}, 1.0$ equiv), dichloroethane $(20.0 \mathrm{~mL})$ and anhydrous DMF $(20.0 \mathrm{~mL})$ under $\mathrm{N}_{2}$. The reaction was kept at room temperature and $\mathrm{POCl}_{3}(274.6 \mu \mathrm{L}, 3.0 \mathrm{mmol}, 3.0$ equiv) was added dropwise via a syringe. The tube was sealed with a teflon-coated screw cap and the reaction solution was heated at $80{ }^{\circ} \mathrm{C}$ for $8 \mathrm{~h}$. The reaction mixture was diluted with $10 \mathrm{~mL}$ of $\mathrm{CH}_{2} \mathrm{Cl}_{2}$ and $10 \mathrm{~mL}$ of $\mathrm{NaOH}$ aqueous solution $(2.0 \mathrm{M})$. The mixture was 
then stirred rapidly for 2 hour. The organic layer was separated, washed with water, dried with $\mathrm{MgSO}_{4}$, and concentrated. The residue was purified by column chromatography on silica gel (ethyl acetate/petroleum ether $=1 / 10$, v/v) to give $\mathbf{4 a}$ as a yellow solid (408 mg, 78\%). ${ }^{1} \mathrm{H}$ NMR (400 MHz, $\left.\mathrm{CDCl}_{3}\right): \delta=7.20-7.26(\mathrm{~m}, 8 \mathrm{H})$, 7.27-7.34 (m, 16H), 8.83 (s, 1H) ppm. $\left.{ }^{13} \mathrm{C} \mathrm{NMR} \mathrm{(100} \mathrm{MHz,} \mathrm{CDCl}_{3}\right): \delta=106.8,121.1$, $121.5,121.9,124.7,125.8,127.0,127.6,127.7,128.27,128.30,128.6,128.7,128.8$, 129.0, 130.16, 130.20, 130.4, 130.7, 130.8, 131.0, 131.9, 132.1, 132.4, 136.1, 136.4, 136.7, 137.0, 137.4, 187.0 ppm. HRMS $\left(\mathrm{ESI}^{+}\right)$: calcd for $\mathrm{C}_{39} \mathrm{H}_{26} \mathrm{NO}[\mathrm{M}+\mathrm{H}]^{+}$524.2014, found 524.2013.

\section{3,4,8,9-Tetrakis(4-(hexyloxy)phenyl)indolizino[6,5,4,3-ija]quinoline-5-carbaldehy} de (4b)

To a Schlenk tube with a magnetic stir bar was added 3,4,8,9-tetrakis(4-(hexyloxy)phenyl)indolizino[6,5,4,3-ija]quinoline 3ai (896 mg, 1.0 mmol, 1.0 equiv), dichloroethane $(20.0 \mathrm{~mL})$ and anhydrous DMF (20.0 mL) under $\mathrm{N}_{2}$. The reaction was kept at room temperature and $\mathrm{POCl}_{3}(274.6 \mu \mathrm{L}, 3.0 \mathrm{mmol}, 3.0$ equiv) was added dropwise via syringe. The tube was sealed with a teflon-coated screw cap and the reaction solution was heated at $80{ }^{\circ} \mathrm{C}$ for $8 \mathrm{~h}$. The reaction mixture was diluted with $10 \mathrm{~mL}$ of $\mathrm{CH}_{2} \mathrm{Cl}_{2}$ and $10 \mathrm{~mL}$ of $\mathrm{NaOH}$ aqueous solution $(2.0 \mathrm{M})$. The mixture was then stirred rapidly for 2 hour. The organic layer was separated, washed with water, dried with $\mathrm{MgSO}_{4}$, and concentrated. The residue was purified by column chromatography on silica gel (ethyl acetate/petroleum ether $=1 / 10$, v/v) to give $\mathbf{4 b}$ as a yellow solid (665 mg, 72\%). ${ }^{1} \mathrm{H}$ NMR $\left(400 \mathrm{MHz}, \mathrm{CDCl}_{3}\right): \delta=0.92(\mathrm{t}, J=6.4 \mathrm{~Hz}$, $12 \mathrm{H}), 1.35-1.36(\mathrm{~m}, 16 \mathrm{H}), 1.47(\mathrm{~m}, 8 \mathrm{H}), 1.76-1.81(\mathrm{~m}, 8 \mathrm{H}), 3.89-3.97(\mathrm{~m}, 8 \mathrm{H})$, 6.77-6.88 (m, 8H), 7.08-7.22 (m, 9H), 7.25-7.26 (m, 1H), 7.33-7.35 (m, 2H), $8.89(\mathrm{~s}$, 1H) ppm. ${ }^{13} \mathrm{C}$ NMR (100 MHz, $\left.\mathrm{CDCl}_{3}\right): \delta=14.2,22.8,25.89,25.92,29.39,29.43$, $31.76,31.78,68.03,68.04,68.1,100.1,106.4,114.3,114.6,114.7,114.9,120.9,121.2$, 121.6, 124.5, 126.1, 127.3, 128.7, 128.9, 129.05, 129.09, 129.5, 130.9, 131.2, 131.36, 131.39, 131.46, 131.49, 131.8, 131.9, 136.1, 158.28, 158.32, 158.4, 158.8, 187.4 ppm. HRMS (ESI ${ }^{+}$: calcd for $\mathrm{C}_{63} \mathrm{H}_{74} \mathrm{NO}_{5}[\mathrm{M}+\mathrm{H}]^{+}$924.5567, found 924.5576. 
(5a)

To a Schlenk tube with a magnetic stir bar was added 3,4,8,9-tetraphenylindolizino[6,5,4,3-ija]quinoline-5-carbaldehyde 4a (93 mg, 0.178 mmol, 1.0 equiv), chloroform (2.0 mL), cyanoacetic acid (90 mg, $1.062 \mathrm{mmol}, 6.0$ equiv) and piperidine (122.2 $\mu \mathrm{L}, 2.492$ mmol, 14.0 equiv) under $\mathrm{N}_{2}$. The tube was sealed with a teflon-coated screw cap and the reaction solution was heated at $80{ }^{\circ} \mathrm{C}$ for $4 \mathrm{~h}$. Then the reaction mixture was diluted with $\mathrm{CH}_{2} \mathrm{Cl}_{2}$ and $\mathrm{AcOH}$ was added to ensure acidity before rinsing with $\mathrm{H}_{2} \mathrm{O}$. The organic layer was separated, washed with water, dried with $\mathrm{MgSO}_{4}$, and concentrated. The residue was purified by column chromatography on silica gel (methanol/dichloromethane $=1 / 50, \mathrm{v} / \mathrm{v})$ to give $\mathbf{5 a}$ as a red solid (95 mg, 90\%). ${ }^{1} \mathrm{H}$ NMR (400 MHz, $d_{6}$-DMSO): $\delta=5.75$ (s, 1H), 7.07 (d, $J$ $=8.0 \mathrm{~Hz}, 1 \mathrm{H}), 7.17(\mathrm{~d}, J=7.6 \mathrm{~Hz}, 1 \mathrm{H}), 7.21-7.41(\mathrm{~m}, 20 \mathrm{H}), 7.48(\mathrm{t}, J=8.0 \mathrm{~Hz}, 1 \mathrm{H})$, 7.57 (s, 1H) ppm. ${ }^{13} \mathrm{C}$ NMR (100 MHz, $d_{6}$-DMSO): $\delta=54.9,105.5,113.4,117.6$, $121.0,121.5,125.3,125.5,125.9,127.5,127.6,127.9,128.15,128.23,128.4,128.65$, 128.73, 128.9, 129.5, 129.6, 129.7, 129.9, 130.3, 130.5, 130.7, 131.0, 132.3, 135.4, 135.7, 136.0, 136.1, 136.4, 145.8, 163.8 ppm. HRMS $\left(\mathrm{ESI}^{+}\right)$: calcd for $\mathrm{C}_{42} \mathrm{H}_{27} \mathrm{~N}_{2} \mathrm{O}_{2}$ $[\mathrm{M}+\mathrm{H}]^{+}$591.2073, found 591.2070.

\section{2-Cyano-3-(3,4,8,9-tetrakis(4-(hexyloxy)phenyl)indolizino $[6,5,4,3-i j a] q u i n o l i n-5-y$} l)acrylic acid (5b)

To a Schlenk tube with a magnetic stir bar was added 2-cyano-3-(3,4,8,9-tetraphenylindolizino[6,5,4,3-ija]quinolin-5-yl)acrylic acid $\mathbf{4 b}$ (165 mg, $0.178 \mathrm{mmol}, 1.0$ equiv), chloroform $(2.0 \mathrm{~mL})$, cyanoacetic acid (90 mg, $1.062 \mathrm{mmol}, 6.0$ equiv) and piperidine (122.2 $\mu \mathrm{L}, 2.492 \mathrm{mmol}, 14.0$ equiv) under $\mathrm{N}_{2}$. The tube was sealed with a teflon-coated screw cap and the reaction solution was heated at $80{ }^{\circ} \mathrm{C}$ for $4 \mathrm{~h}$. Then the reaction mixture was diluted with $\mathrm{CH}_{2} \mathrm{Cl}_{2}$ and $\mathrm{AcOH}$ was added to ensure acidity before rinsing with $\mathrm{H}_{2} \mathrm{O}$. The organic layer was separated, washed with water, dried with $\mathrm{MgSO}_{4}$, and concentrated. The residue was purified by 
column chromatography on silica gel (methanol/dichloromethane $=1 / 50, \mathrm{v} / \mathrm{v}$ ) to give 5b as a red solid (150 mg, 85\%). ${ }^{1} \mathrm{H}$ NMR (400 MHz, $\left.\mathrm{CDCl}_{3}\right): \delta=0.82-0.85$ (m, 3H), 0.90-0.94 (m, 9H), 1.27-1.37 (m, 16H), 1.43-1.48 (m, 8H), 1.75-1.81 (m, 8H), 3.92-3.98 (m, 8H), 6.80-6.89 (m, 8H), 7.06-7.12 (m, 6H), $7.22(\mathrm{~d}, J=8.8 \mathrm{~Hz}, 2 \mathrm{H})$, 7.25-7.27 (m, 2H), 7.34-7.36 (m, 2H), $7.58(\mathrm{~s}, 1 \mathrm{H}), 7.79(\mathrm{~s}, 1 \mathrm{H}) \mathrm{ppm} .{ }^{13} \mathrm{C}$ NMR $(100$ $\left.\mathrm{MHz}, \mathrm{CDCl}_{3}\right): \delta=14.2,22.8,25.9,29.41,29.43,29.5,31.8,68.0,68.07,68.12,68.5$, $94.1,107.0,114.3,114.4,114.6,114.8,115.4,117.1,121.6,122.1,125.0,126.5,127.2$, $127.8,128.2$, 128.6, 129.0, 130.4, 131.0, 131.1, 131.3, 131.4, 131.5, 131.56, 131.58, 131.9, 132.4, 137.6, 149.4, 158.4, 158.7, 159.4 ppm. HRMS $\left(\mathrm{ESI}^{+}\right)$: calcd for $\mathrm{C}_{66} \mathrm{H}_{75} \mathrm{~N}_{2} \mathrm{O}_{6}[\mathrm{M}+\mathrm{H}]^{+}$991.5625, found 991.5620.

\section{2-(4-Oxo-5-((3,4,8,9-tetrakis(4-(hexyloxy)phenyl)indolizino[6,5,4,3-ija]quinolin-5- yl)methylene)-2-thioxothiazolidin-3-yl)acetic acid (5c)}

To a Schlenk tube with a magnetic stir bar was added 2-cyano-3-(3,4,8,9-tetraphenylindolizino[6,5,4,3-ija]quinolin-5-yl)acrylic acid $\mathbf{4 b}$ (165 mg, $0.178 \mathrm{mmol}, 1.0$ equiv), ethanol (4.0 mL), rhodanine-3-acetic acid (37 mg, $0.196 \mathrm{mmol}, 1.1$ equiv) and piperidine (18 $\mu \mathrm{L}, 0.196 \mathrm{mmol}, 1.1$ equiv) under $\mathrm{N}_{2}$. The tube was sealed with a teflon-coated screw cap and the reaction solution was reflux for $4 \mathrm{~h}$. Then the reaction mixture was diluted with $\mathrm{CH}_{2} \mathrm{Cl}_{2}$ and $\mathrm{AcOH}$ was added to ensure acidity before rinsing with $\mathrm{H}_{2} \mathrm{O}$. The organic layer was separated, washed with water, dried with $\mathrm{MgSO}_{4}$, and concentrated. The residue was purified by column chromatography on silica gel (methanol/dichloromethane $=1 / 50, \mathrm{v} / \mathrm{v})$ to give $\mathbf{5} \mathbf{c}$ as a red solid (156 mg, 80\%). ${ }^{1} \mathrm{H}$ NMR (400 MHz, $\left.\mathrm{CDCl}_{3}\right): \delta=0.91-0.92(\mathrm{~m}, 12 \mathrm{H})$, 1.36-1.48 (m, 24H), 1.75-1.85 (m, 8H), 3.91-4.02 (m, 8H), 4.77 (s, 2H), 6.80-6.91 (m, 9H), 7.02-7.11 (m, 7H), 7.18-7.24 (m, 3H), 7.31-7.33 (m, 2H) ppm. ${ }^{13} \mathrm{C}$ NMR (100 $\left.\mathrm{MHz}, \mathrm{CDCl}_{3}\right): \delta=14.2,22.8,25.90,25.92,25.94,29.37,29.43,29.5,31.78,31.83$, 68.0, 68.06, 68.07, 68.5, 106.4, 114.4, 114.6, 114.7, 115.3, 116.40, 116.43, 121.1, 121.6, 124.7, 126.6, 126.9, 128.1, 128.5, 128.8, 129.0, 129.78, 129.81, 130.0, 130.3, 130.7, 131.1, 131.2, 131.5, 131.7, 131.75, 131.80, 132.2, 136.0, 158.27, 158.31, 158.6, 159.2, 166.3, 187.5, 193.4 HRMS $\left(\mathrm{ESI}^{+}\right)$: calcd for $\mathrm{C}_{68} \mathrm{H}_{77} \mathrm{~N}_{2} \mathrm{O}_{7} \mathrm{~S}_{2}[\mathrm{M}+\mathrm{H}]^{+}$1097.5172, 
found 1097.5180.

\section{Cell fabrication}

The photoanode used was a $\mathrm{TiO}_{2}$ thin film $(13 \mu \mathrm{m}$ of $20 \mathrm{~nm}$ particles as the absorbing layer and $5 \mu \mathrm{m}$ of $400 \mathrm{~nm}$ particles as the scattering layer) coated on an FTO glass substrate with dimensions of $0.4 \times 0.4 \mathrm{~cm}^{2}$, and a film thickness was measured by means of a profilometer (E-MC-S24B, Japan.). The $\mathrm{TiO}_{2}$ thin film was dipped into the solution containing $5.0 \times 10^{-4} \mathrm{M}$ dye sensitizers in chloroform/ethanol (4:6, v/v) at room temperature for $24 \mathrm{~h}$. After rinsing with $\mathrm{EtOH}$, the photoanode was adhered with polyimide tape (Parafilm, Sigma-Aldrich) with $30 \mu \mathrm{m}$ thickness and a square aperture of $0.36 \mathrm{~cm}^{2}$ was placed on the top of the counter electrode, which were tightly clipped together to form a cell. Electrolyte was then injected into the seam between the two electrodes. The electrolyte was composed of $1.0 \mathrm{M}$ 1,2-dimethy-3-propyl imidazolium iodide (DMPII), 0.1 M lithium iodide (LiI), 0.05 M iodine $\left(\mathrm{I}_{2}\right)$, and $0.5 \mathrm{M}$ 4-tert-butylpyridine dissolved in acetonitrile.

Photoelectro-chemical characterizations on the solar cells were performed by using an Oriel Class AAA solar simulator (Oriel 94023A, Newport Corp.). Photo-current-voltage characteristics of the DSSCs were recorded with a potentiostat/galvanostat (Keithley Series 2400 SourceMeter, Keithley Instruments, Inc.) at a light intensity of $100 \mathrm{~mW} \mathrm{~cm}^{2}$ calibrated by an Oriel reference solar cell (Oriel 91150V, Newport Corp.). The monochromatic quantum efficiency was recorded through a monochromator (Oriel 77890, Newport Corp.) under short-circuit condition.

\section{Electrochemical and photochemical properties of dyes}

Cyclic voltammetry (CV) measurements were performed on LK2005A at a scan rate of $50 \mathrm{mV} \mathrm{s}^{-1}$ with a platinum disk as a working electrode, a platinum wire as counter electrode and an $\mathrm{Ag} / \mathrm{Ag}^{+}\left(0.01 \mathrm{M}\right.$ of $\mathrm{AgNO}_{3}$ in acetonitrile) as a pseudo-reference electrode calibrated against ferrocene $(\mathrm{Fc})$ couple and converted to NHE by addition of $630 \mathrm{mV}$. CVs were recorded in $0.1 \mathrm{M} n-\mathrm{Bu}_{4} \mathrm{NPF}_{6} / \mathrm{CH}_{2} \mathrm{Cl}_{2}$ for $\mathbf{5 a}, \mathbf{5 b}$ and $\mathbf{5 c}$. 


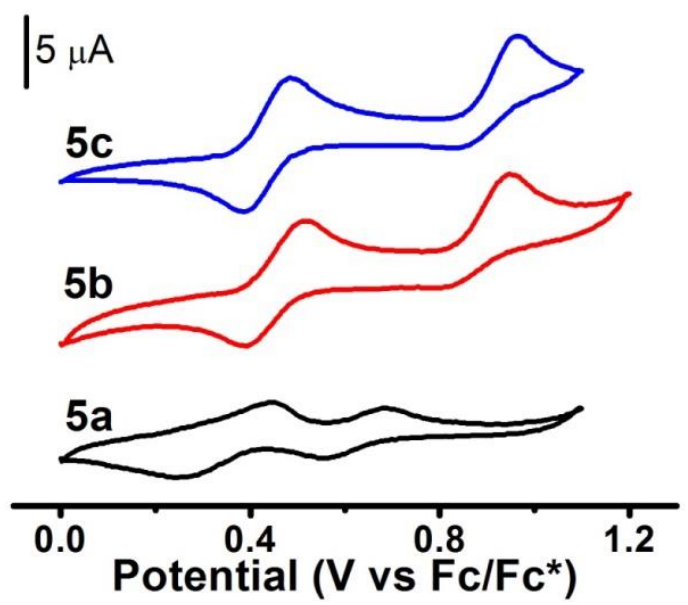

Figure S1. Cyclic voltammograms of $\mathbf{5 a}, \mathbf{5 b}$ and $\mathbf{5 c}$.

Table S2. Absorption and Electrochemical Parameters for 5a, 5b, and $\mathbf{5 c}$.

\begin{tabular}{|c|c|c|c|c|}
\hline Dye & $\lambda_{\max }\left(\varepsilon \times 10^{4} \mathrm{M}^{-1} \mathrm{~cm}^{-1}\right) / \mathrm{nm}^{a}$ & $E_{1 / 2}^{o x} / \mathrm{V}^{b}$ & $E_{0-0} / \mathrm{eV}^{c}$ & $E_{1 / 2}^{o x *} / \mathrm{V}^{d}$ \\
\hline $\mathbf{5 a}$ & $475(2.80), 387(1.52)$ & $0.86,1.14$ & 2.34 & -1.48 \\
\hline $\mathbf{5 b}$ & $482(2.82), 391(1.59)$ & $0.96,1.40$ & 2.33 & -1.37 \\
\hline $\mathbf{5 c}$ & $531(4.14), 417(1.83)$ & $0.95,1.42$ & 2.12 & -1.17 \\
\hline
\end{tabular}

${ }^{a}$ Absorption maximum in a $\mathrm{CH}_{2} \mathrm{Cl}_{2}$ solution $\left(1.0 \times 10^{-5} \mathrm{M}\right) .{ }^{b}$ Oxidation potential in a $\mathrm{CH}_{2} \mathrm{Cl}_{2}$ solution containing $0.1 \mathrm{M}\left(n-\mathrm{C}_{4} \mathrm{H}_{9}\right)_{4} \mathrm{NPF}_{6}$ with a scan rate of $50 \mathrm{mV} \mathrm{s}^{-1}$ (vs NHE). ${ }^{c} E_{0-0}$ was determined from the onset of absorption spectrum. ${ }^{d} E_{1 / 2}^{o x *}=E_{1 / 2}^{o x}-$ $E_{0-0}$.

Table S3. Photovoltaic Parameters of DSSCs under Full Sunlight Illumination (AM $\left.1.5 \mathrm{G}, 100 \mathrm{~mW} \mathrm{~cm}^{-2}\right)^{a}$

\begin{tabular}{|c|c|c|c|c|}
\hline Dye & $J_{\mathrm{sc}} / \mathrm{mA} \mathrm{cm}^{-2}$ & $V_{\mathrm{oc}} / \mathrm{V}$ & $\mathrm{FF}$ & $\eta / \%$ \\
\hline $\mathbf{5 a}$ & 6.45 & 0.635 & 0.633 & 2.6 \\
\hline $\mathbf{5 b}$ & 9.06 & 0.649 & 0.663 & 3.9 \\
\hline $\mathbf{5 c}$ & 14.45 & 0.662 & 0.639 & 6.1 \\
\hline $\mathbf{N 7 1 9}$ & 18.77 & 0.760 & 0.692 & 9.9 \\
\hline
\end{tabular}

${ }^{a}$ Performance of DSSCs measured in a $0.4 \times 0.4 \mathrm{~cm}^{2}$ working area on a FTO substrate 
at room temperature. Dyes were maintained at $0.5 \mathrm{mM}$ in chloroform/ethanol (4:6, v/v) for 5a, 5b, 5c and N719. Electrolyte: LiI (0.1 M), $\mathrm{I}_{2}(0.05 \mathrm{M})$, and DMPII (1.0 M) and 4-tert-butylpyridine $(0.5 \mathrm{M})$ in acetonitrile.

\section{References}

(1) (a) Mio, M. J.; Kople, L. C.; Braun, J. B.; Gadzikwa, T. L.; Hull, K. L.; Brisbois, R. G.; Markworth, C. J.; Grieco, P. A. Org. Lett. 2002, 4, 3199-3202. b) Park, K.; Bae, G.; Moon, J.; Choe, J.; Song, K. H.; Lee, S. J. Org. Chem. 2010, 75, 6244-6251.

(2) Delcamp, J. H.; Yella, A.; Holcombe, T. W.; Nazeeruddin, M. K.; Grätzel, M. Angew. Chem. Int. Ed. 2013, 52, 376-380.

(3) Osako, T.; Uozumi, Y. Org. Lett. 2014, 16, 5866-5869. 
X. Copies of ${ }^{1} \mathrm{H}$ and ${ }^{13} \mathrm{C}$ NMR spectra

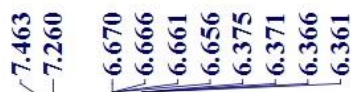

$\underset{\substack{\infty \\ \text { i }}}{i}$
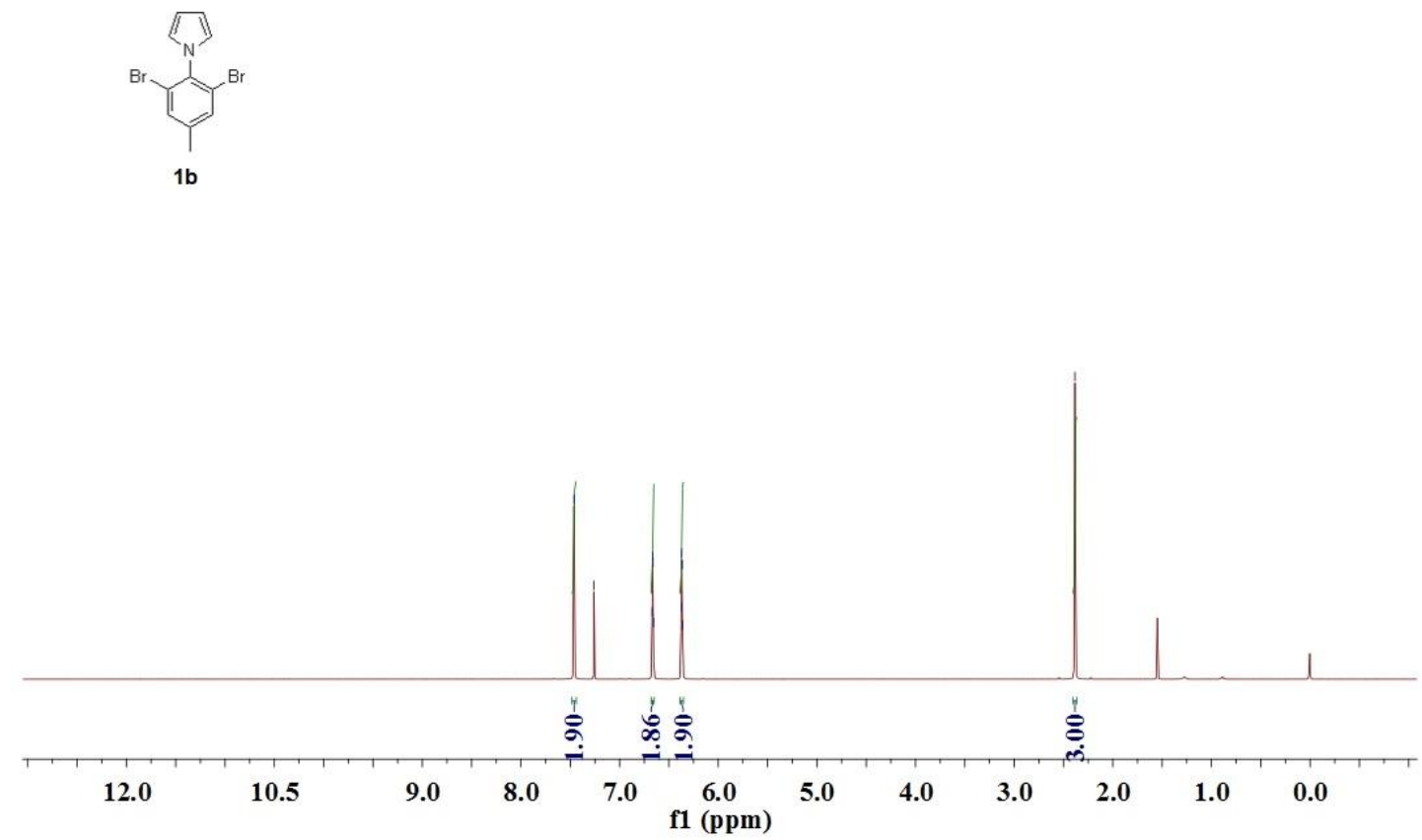

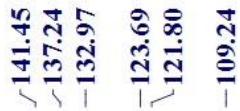

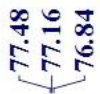

$\stackrel{1}{\stackrel{1}{*}}$

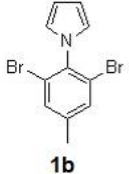

$1 \mathrm{~b}$

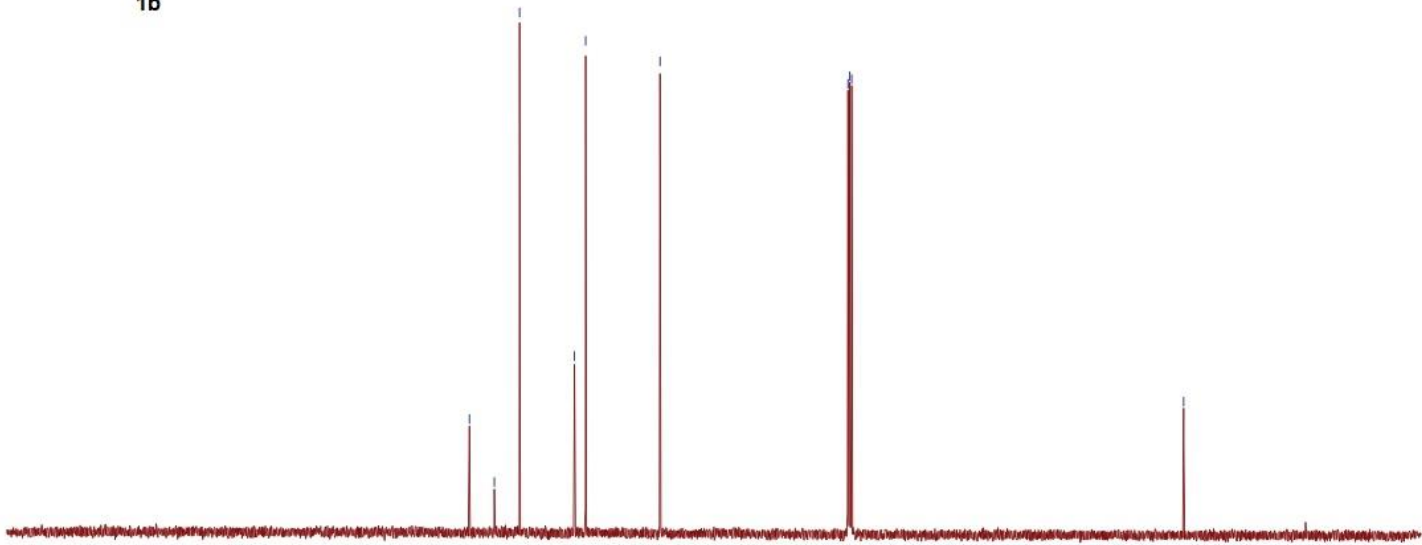

210

190

170

150

130

11

$\begin{array}{llllllllll}90 & 80 & 70 & 60 & 50 & 40 & 30 & 20 & 10 & 0\end{array}$ f1 (ppm) 


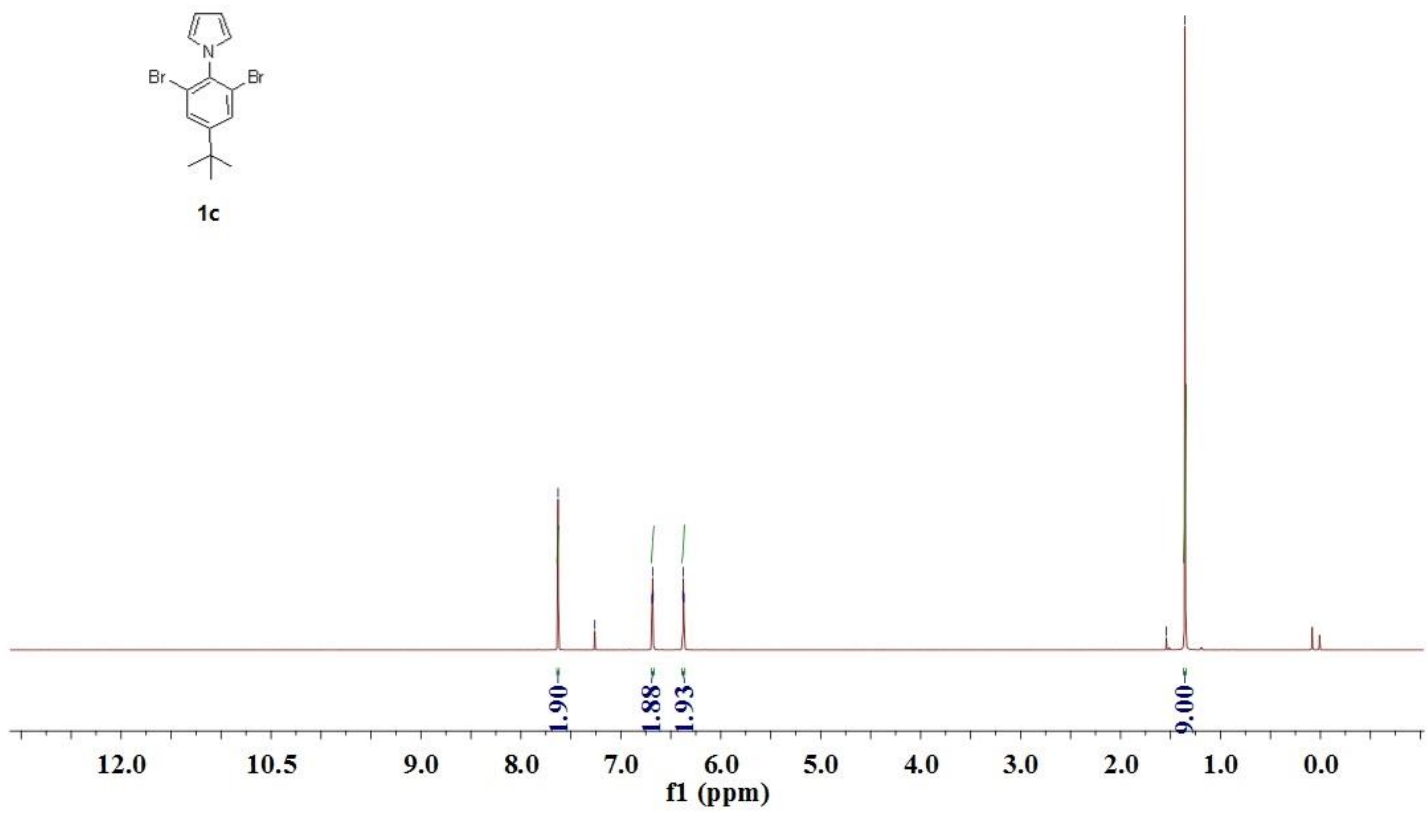

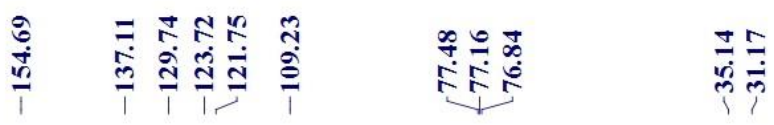

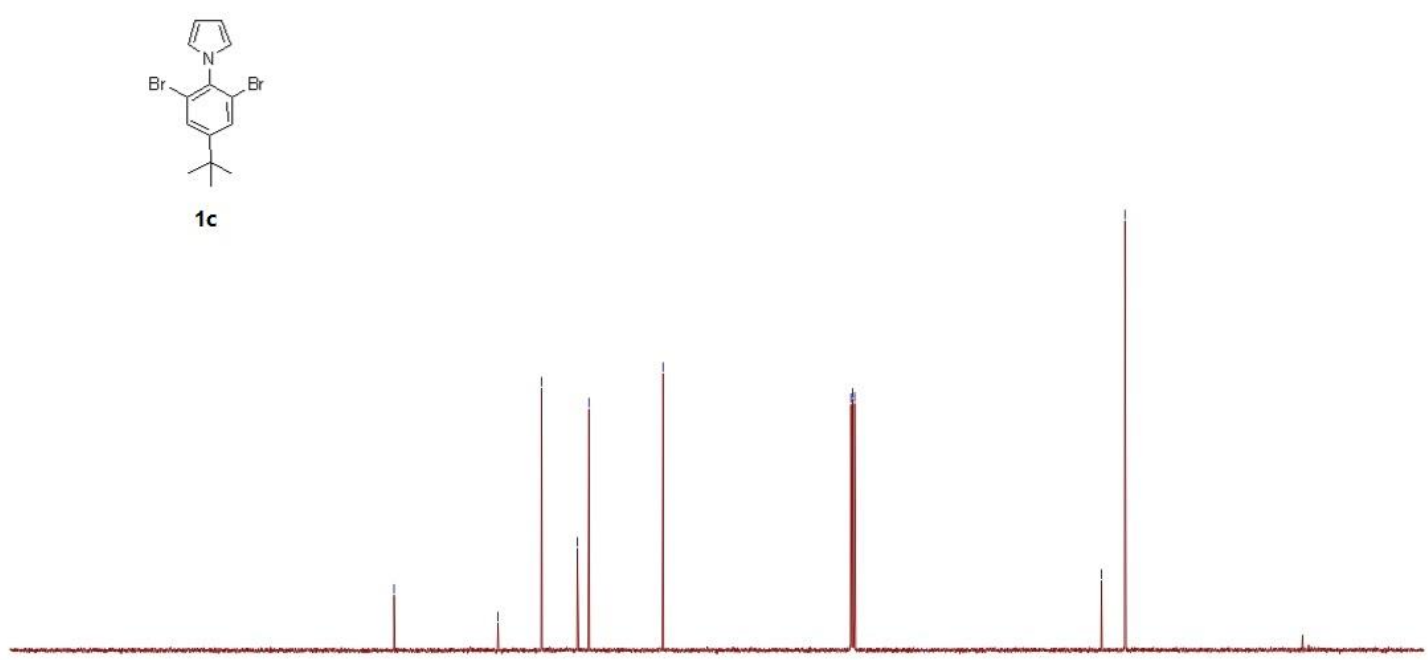

$\begin{array}{lllllllllllllllll}210 & 190 & 170 & 150 & 130 & 110 & 90 & 80 & 70 & 60 & 50 & 40 & 30 & 20 & 10 & 0\end{array}$ 

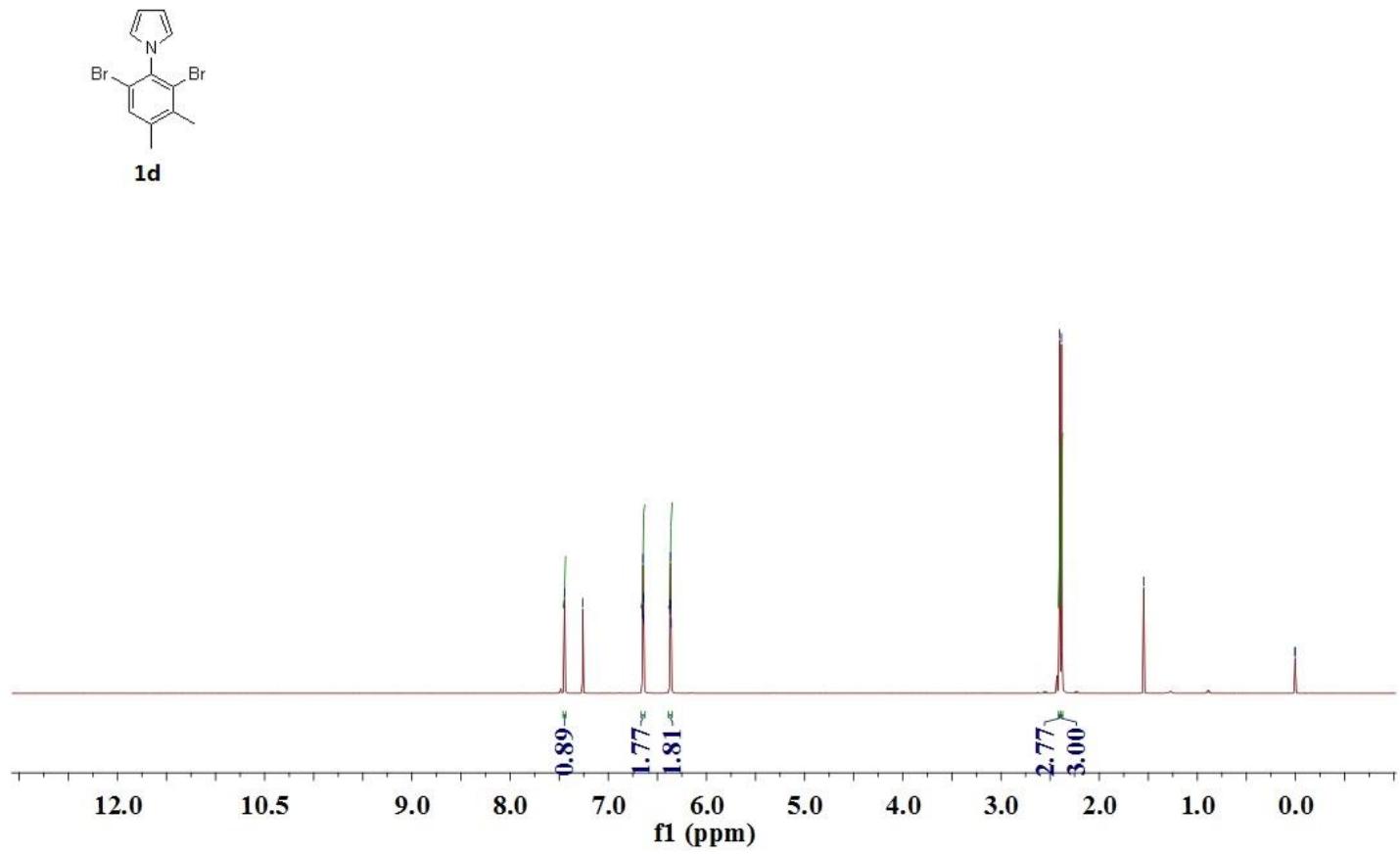

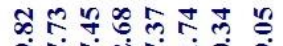

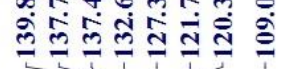

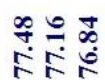

ฺุ?

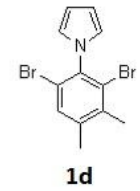

年兵

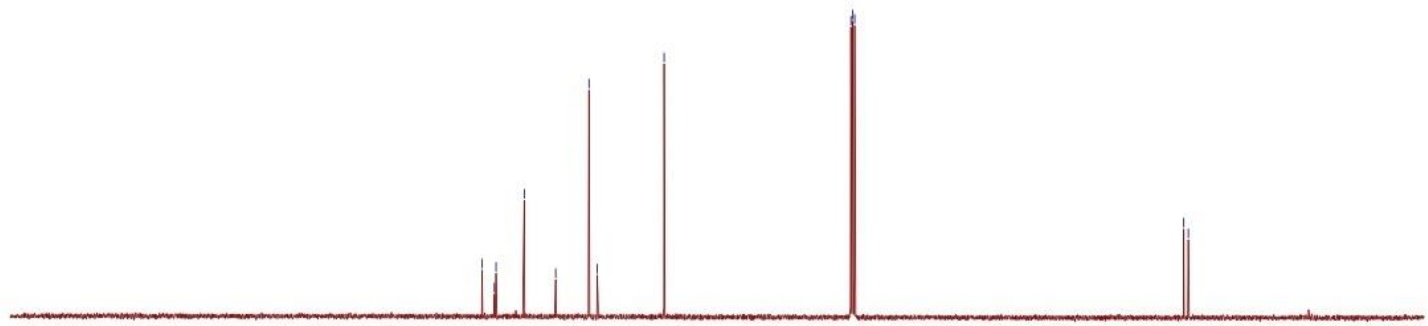

$\begin{array}{llllllllllllllll}210 & 190 & 170 & 150 & 130 & 110 & 90 & 80 & 70 & 60 & 50 & 40 & 30 & 20 & 10 & 0\end{array}$ f1 (ppm) 


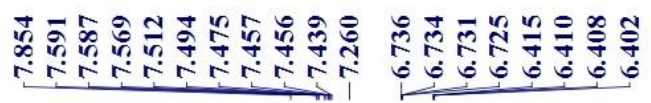
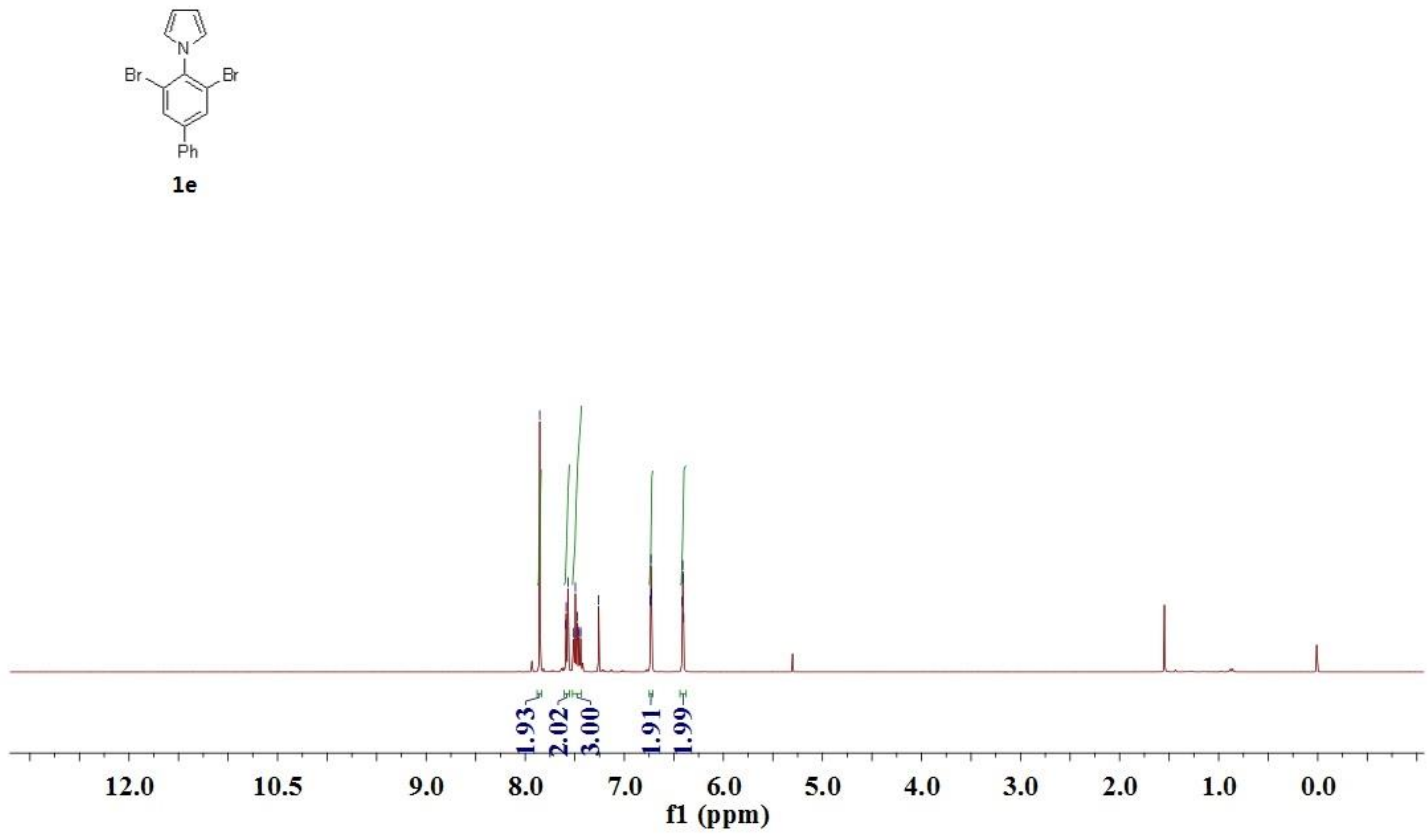

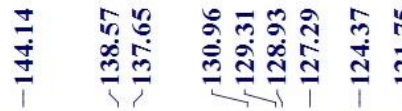

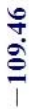

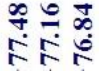

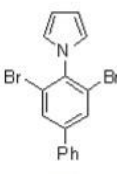

$1 e$

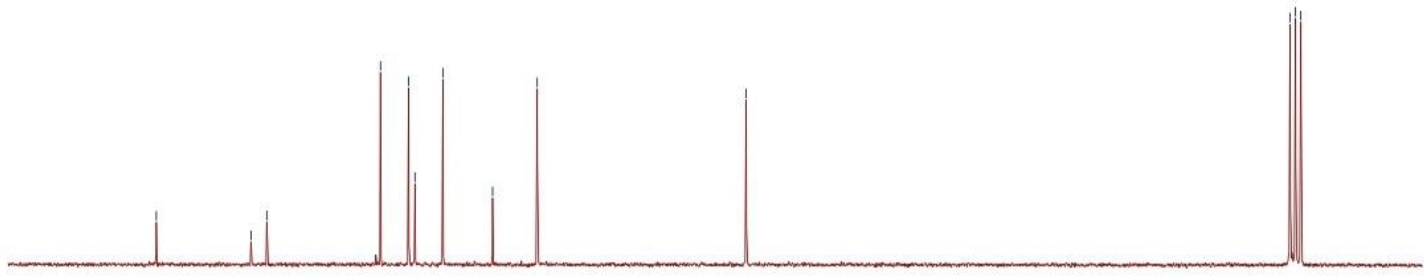

$\begin{array}{lllllllll}150 & 145 & 140 & 135 & 130 & 125 & 120 & 115 & \begin{array}{l}110 \\ \text { f1 }(\mathrm{ppm})\end{array}\end{array}$

$\begin{array}{llllllll}105 & 100 & 95 & 90 & 85 & 80 & 75 & 70\end{array}$ 


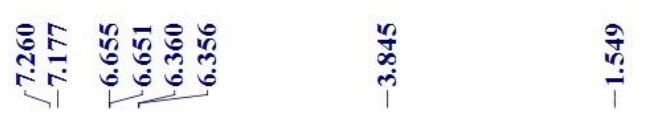

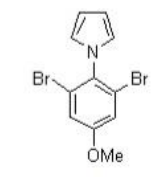

$1 f$

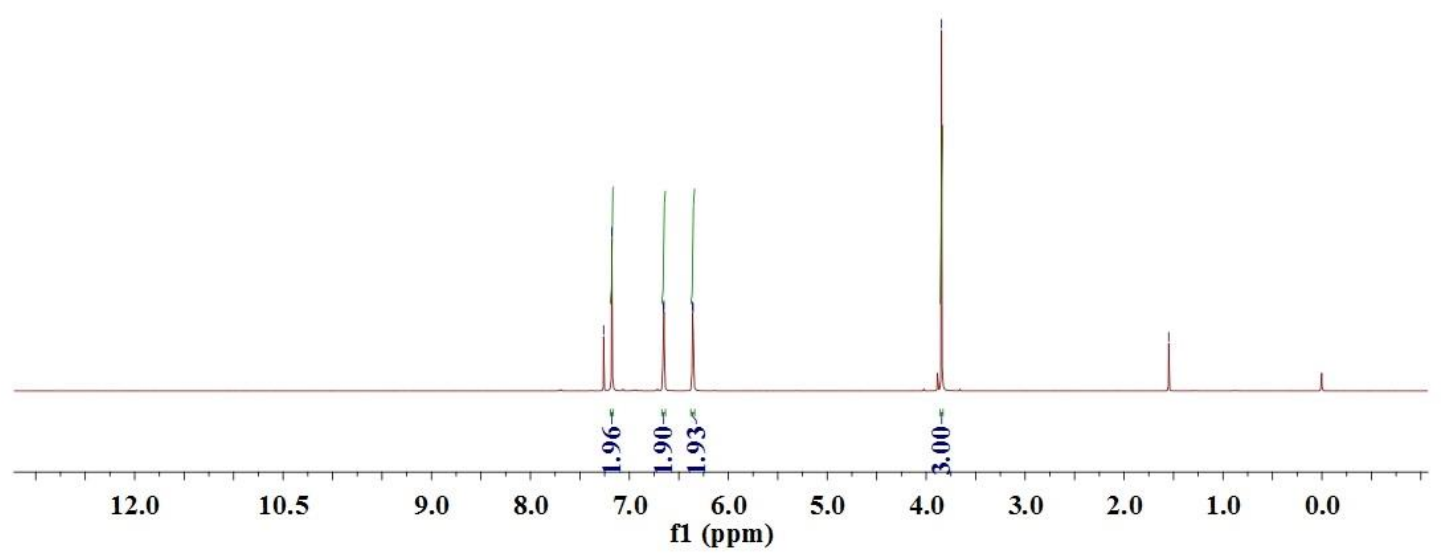

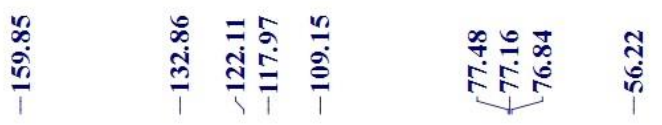

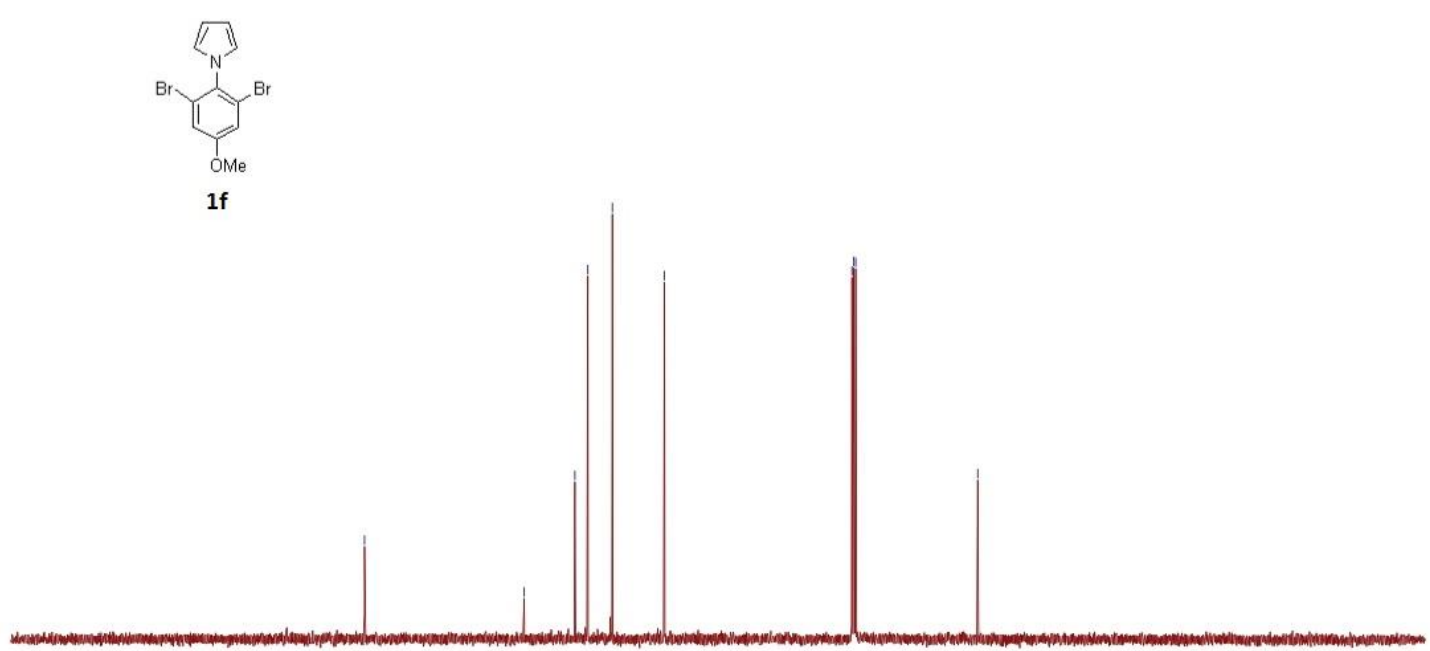

$\begin{array}{lllllllllllllllll}210 & 190 & 170 & 150 & 130 & 110 & 90 & 80 & 70 & 60 & 50 & 40 & 30 & 20 & 10 & 0\end{array}$ 


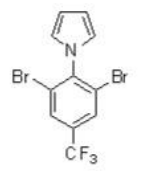

$1 \mathrm{~g}$
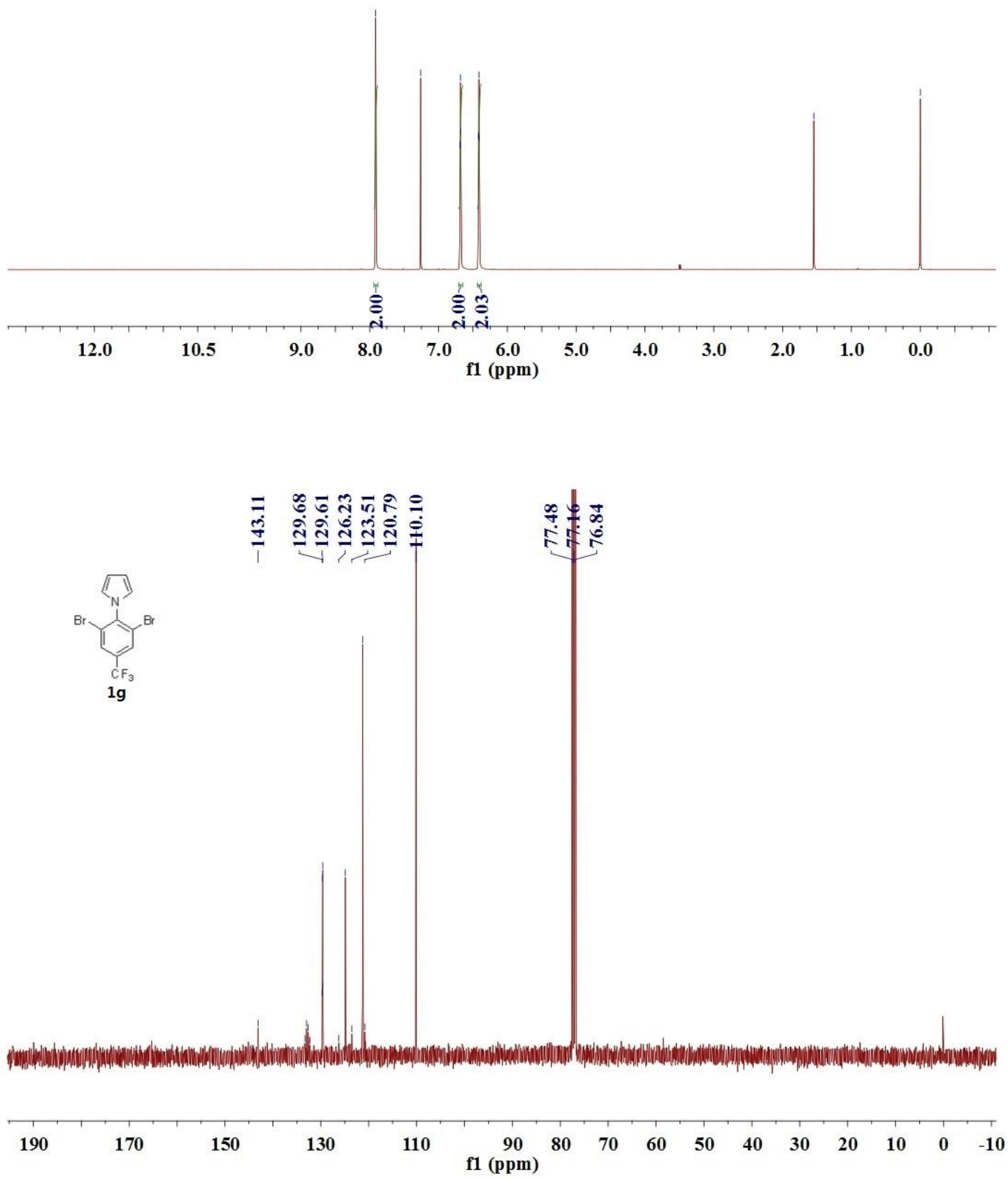


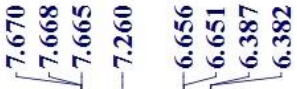

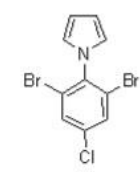

1h

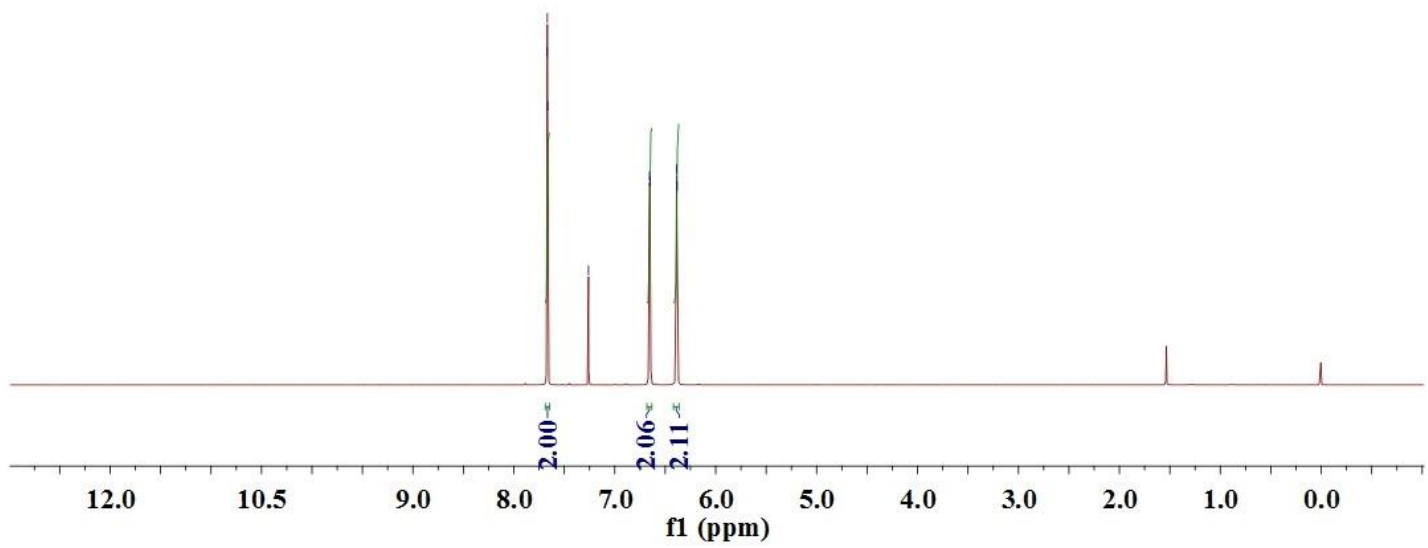

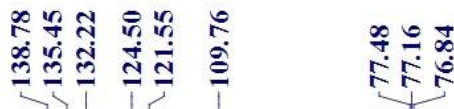

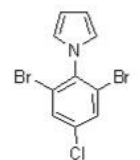

1h

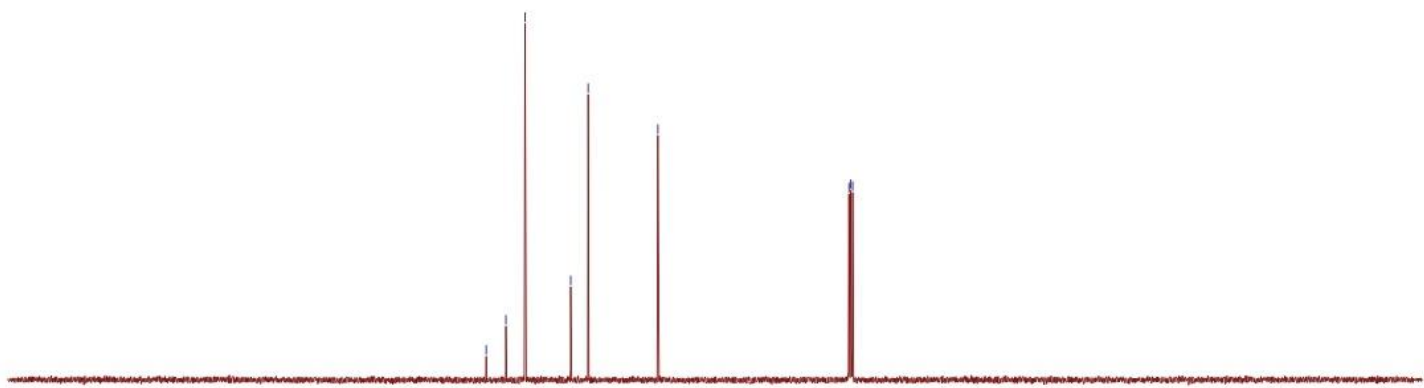

$210 \quad 190$

150

130

110

f1 (ppm) 


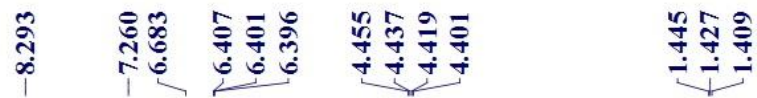
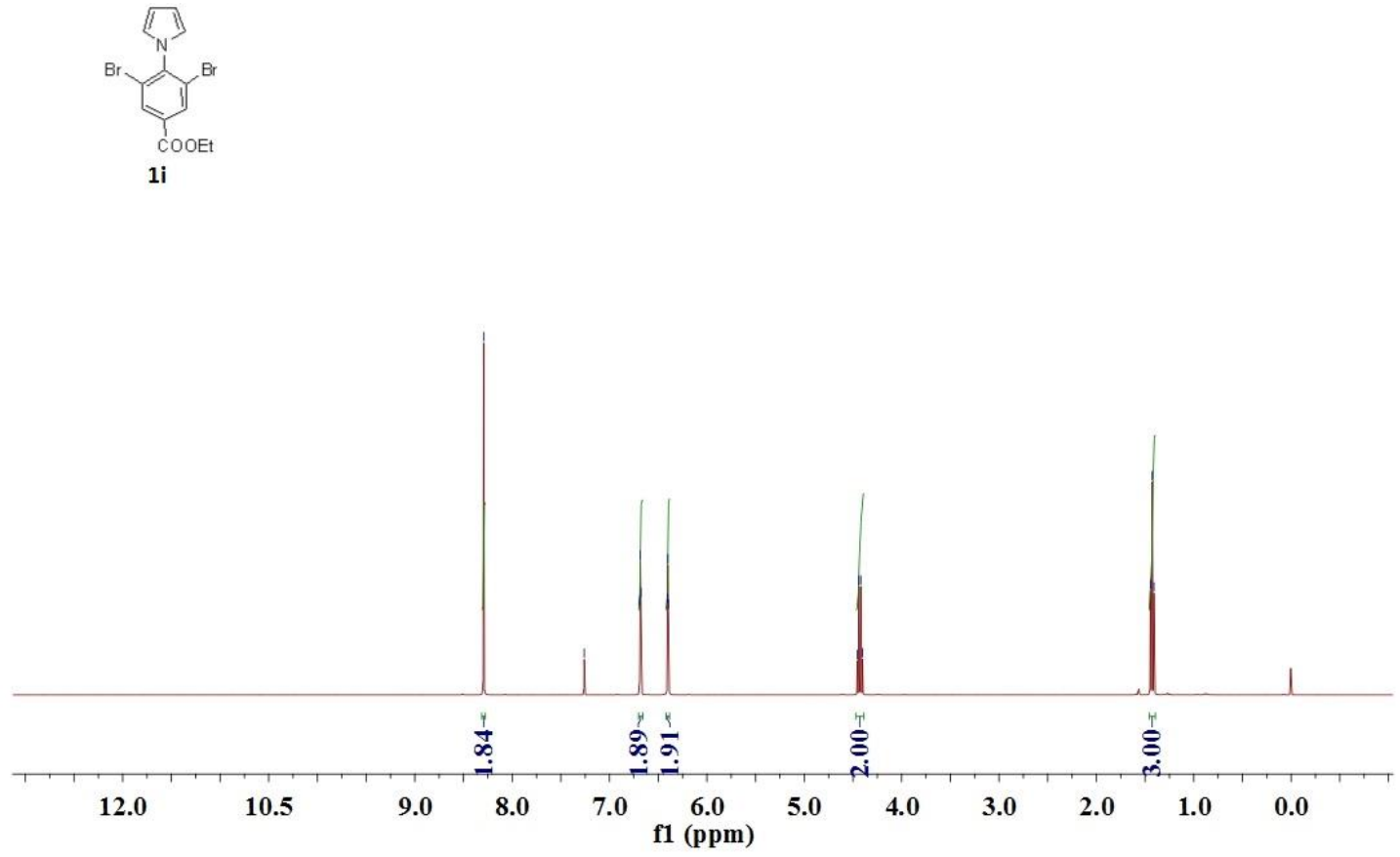

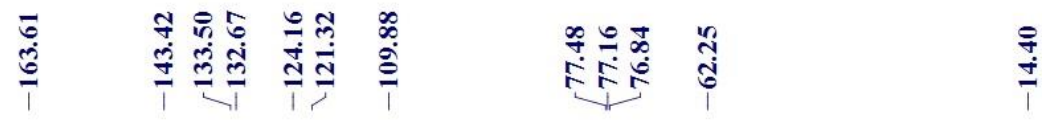

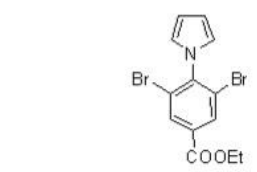

$1 i$

$\underset{+}{\dot{q}}$

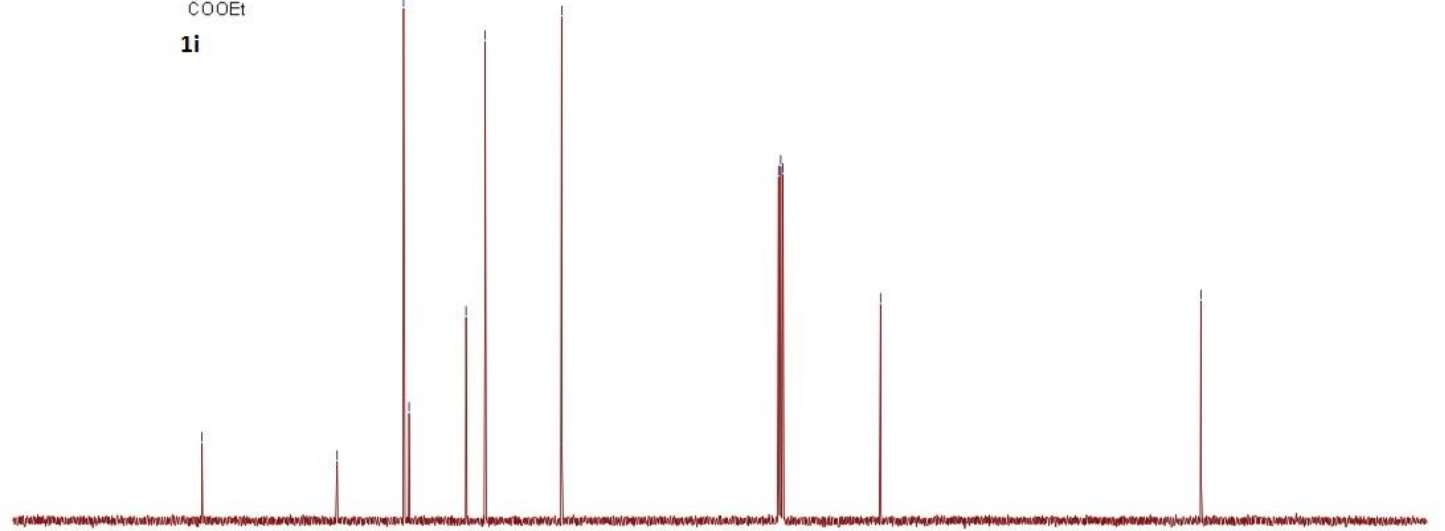

190

170

150

130

110

$\begin{array}{cc}90 & 80 \\ \text { f1 }(\mathrm{ppm})\end{array}$ 


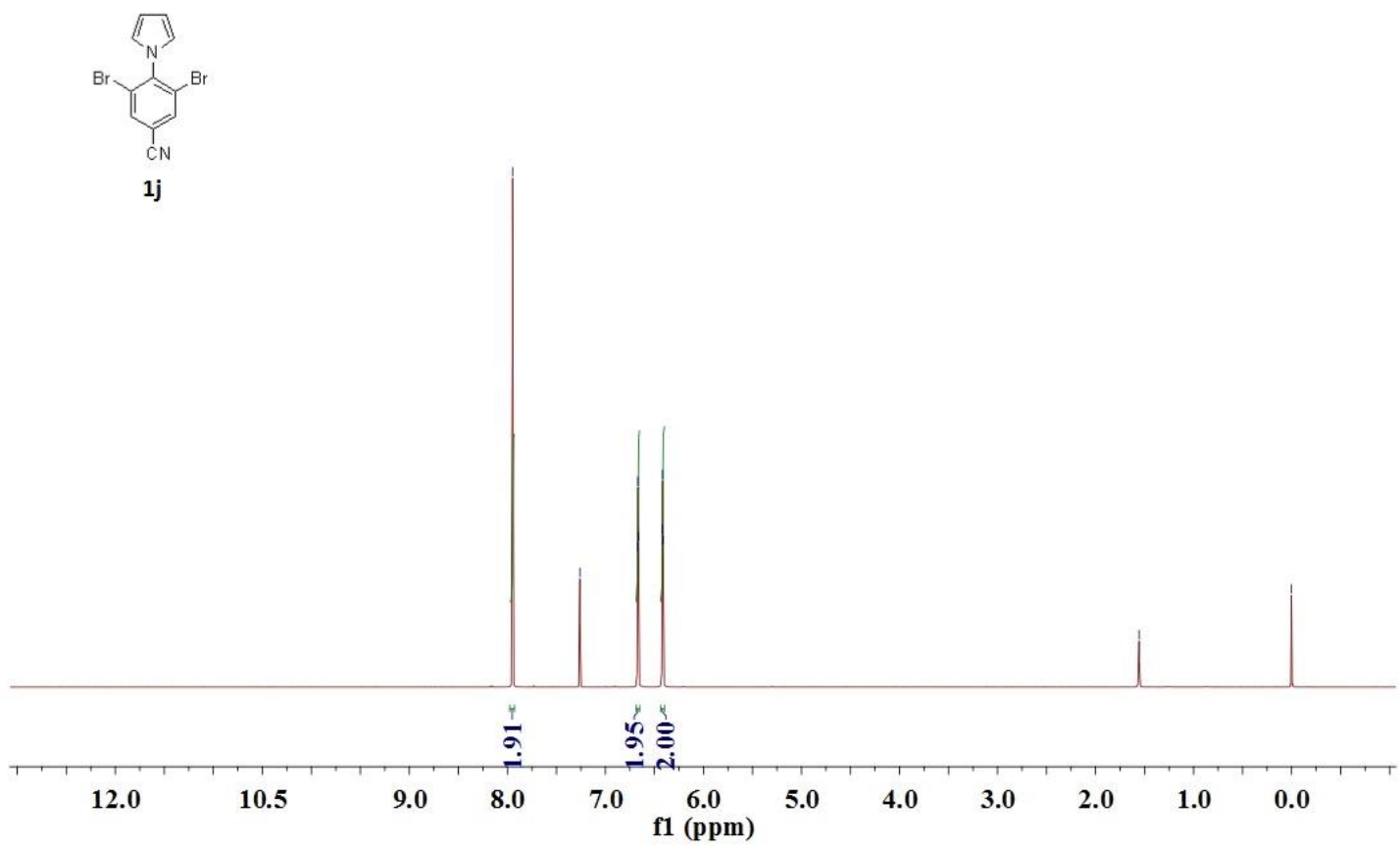

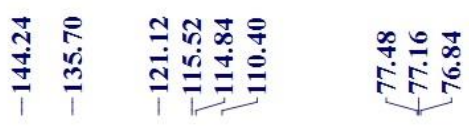

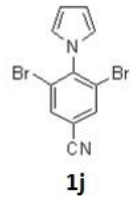

1j
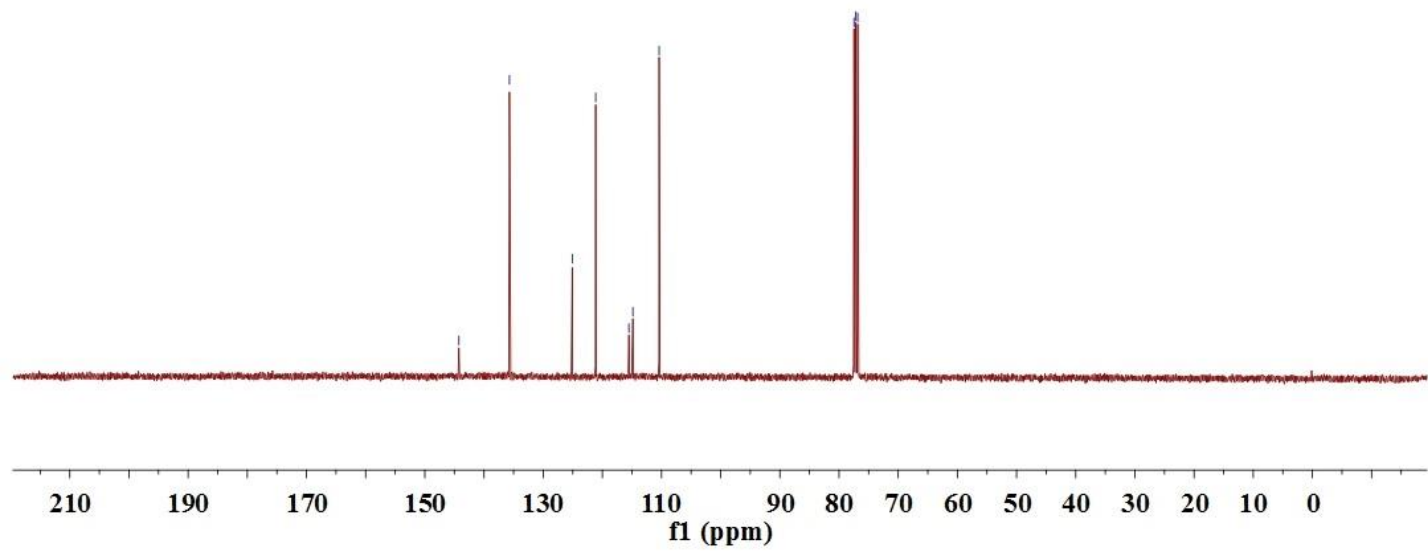


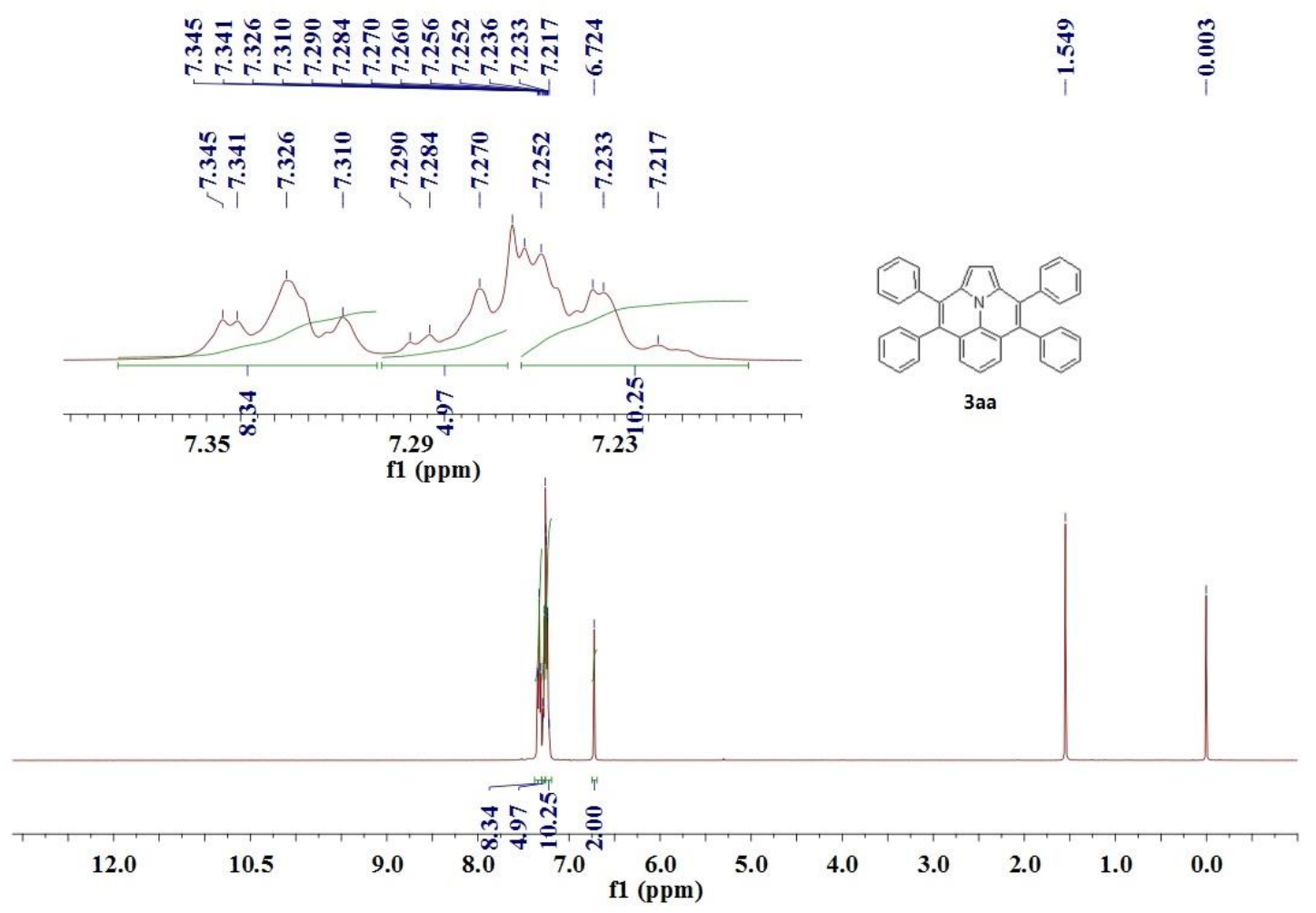

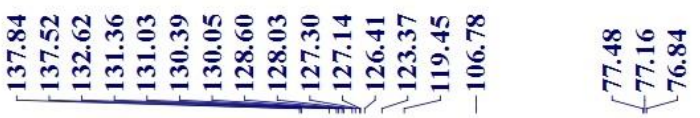
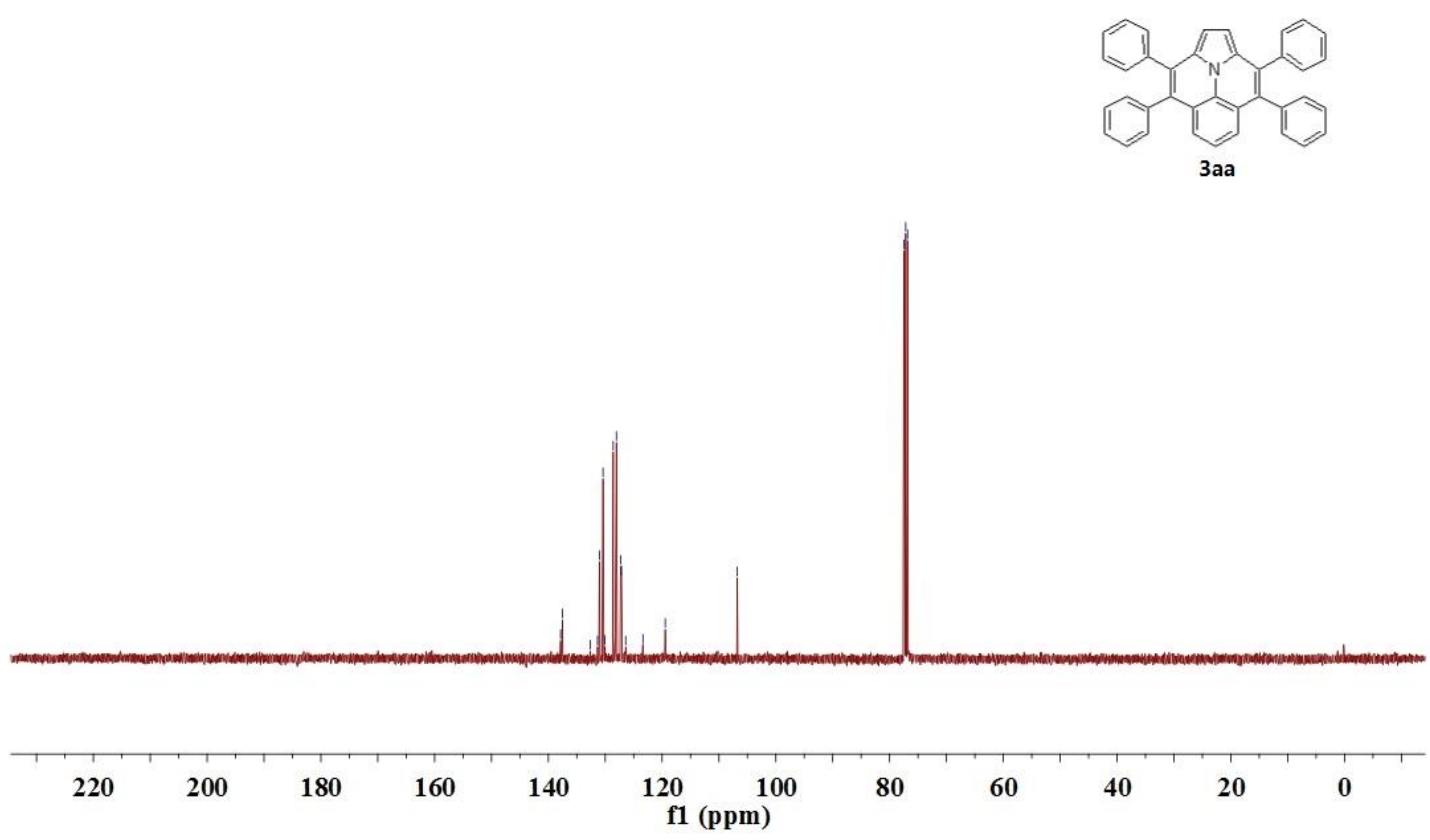

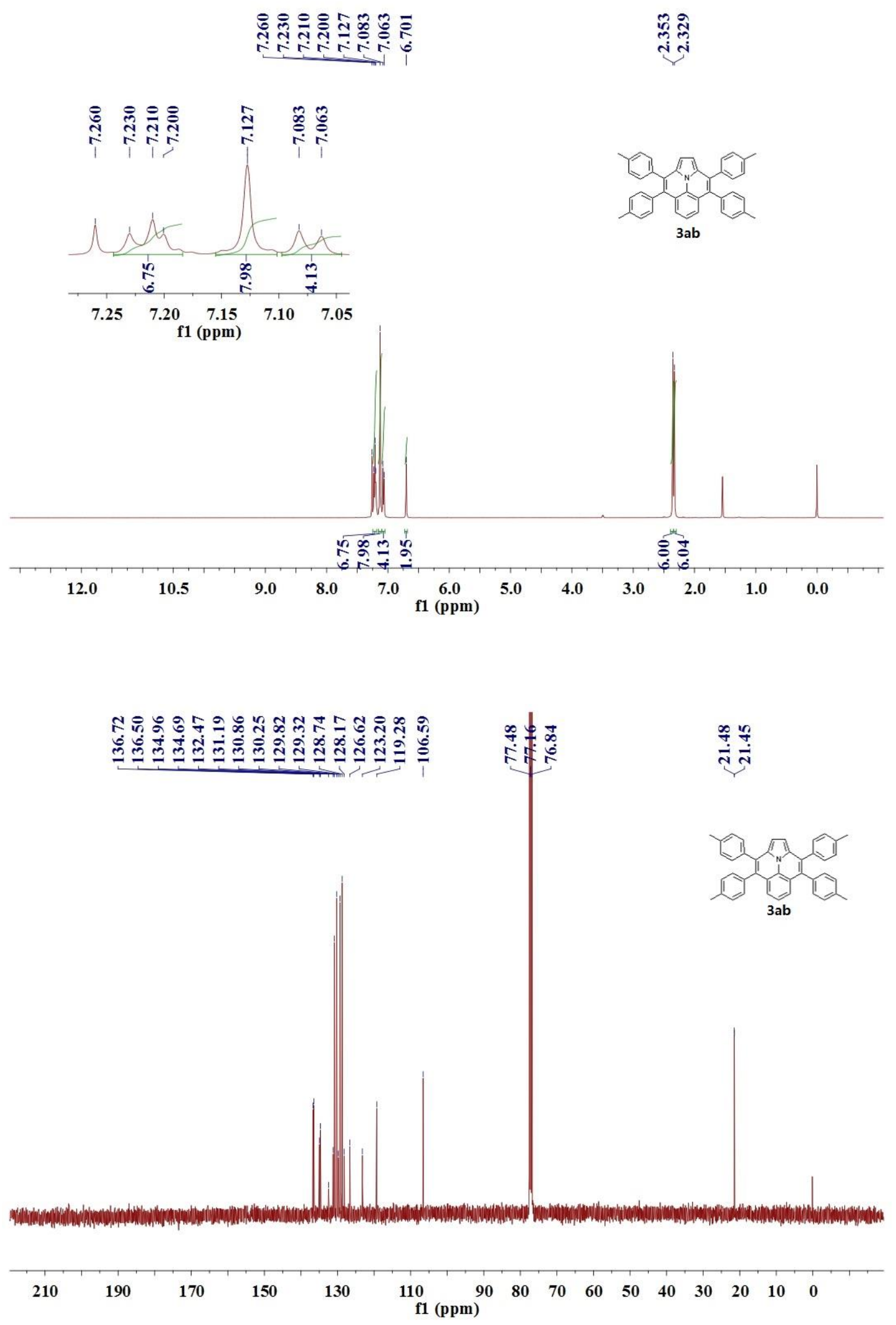


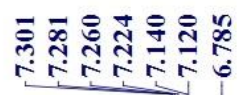
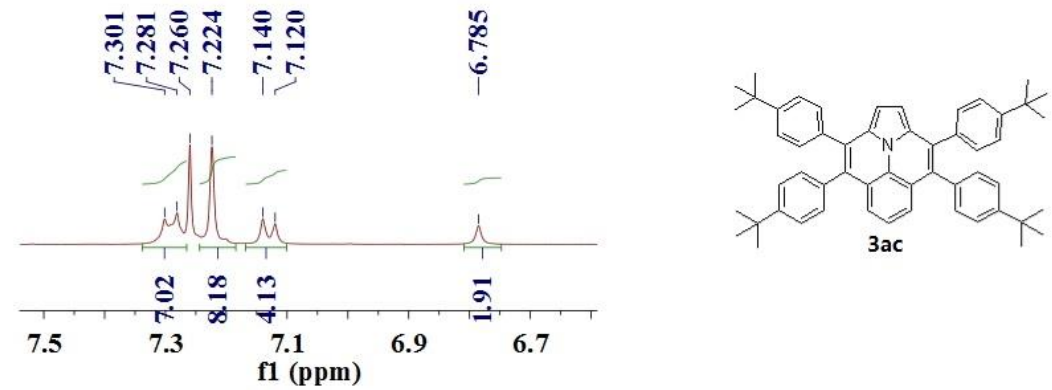

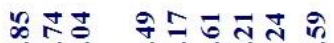

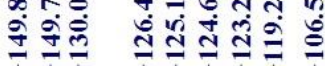

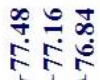

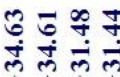

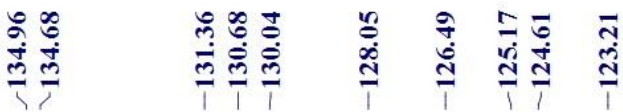
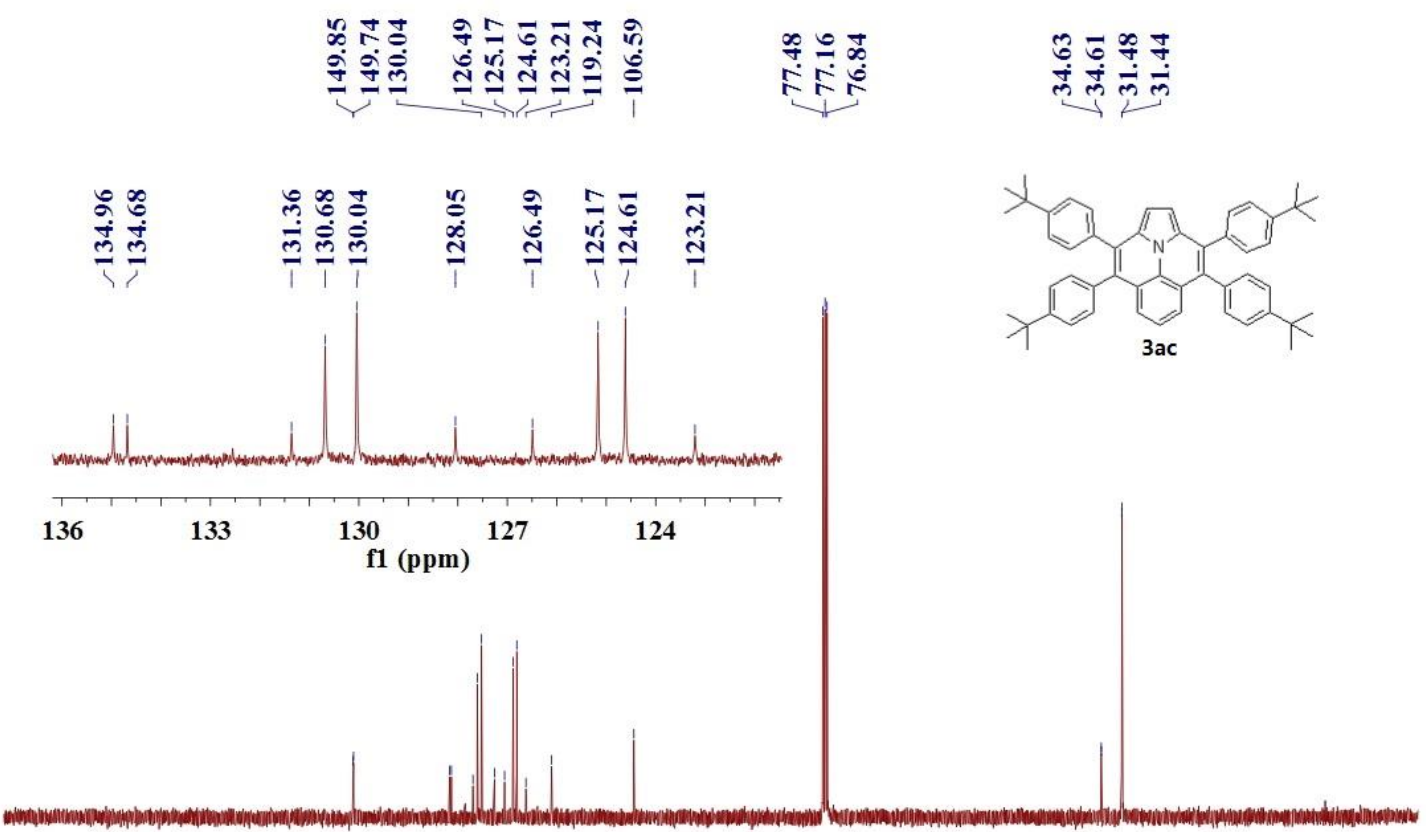

要

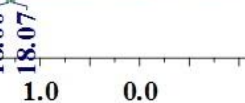

3ac

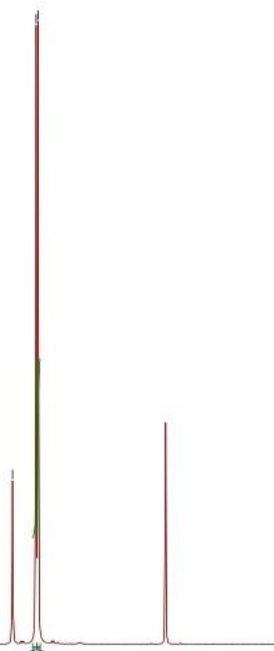

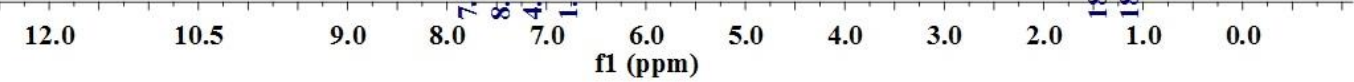




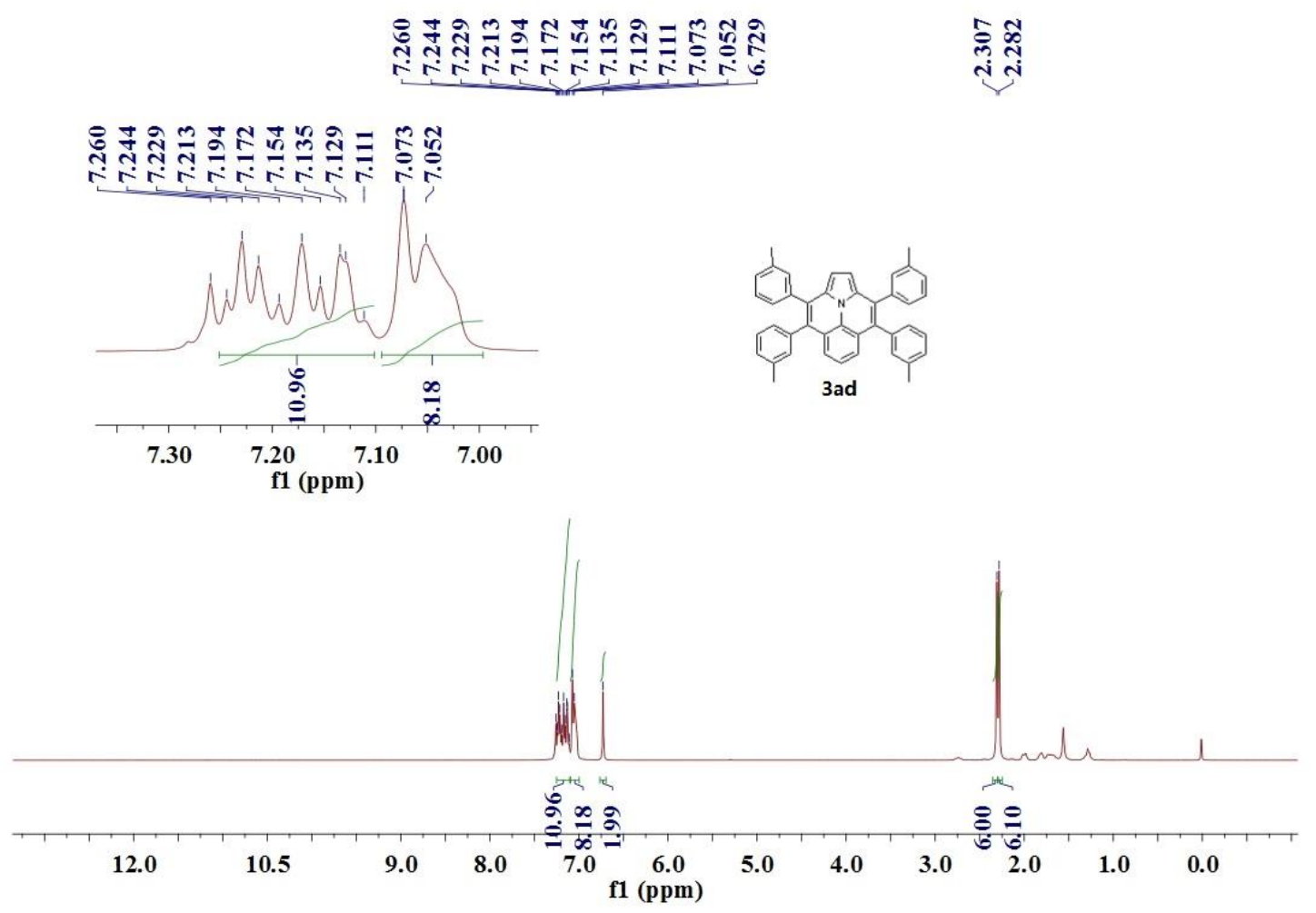

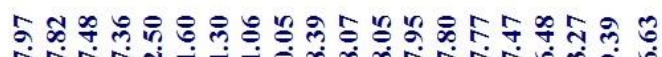

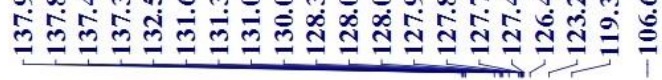

i

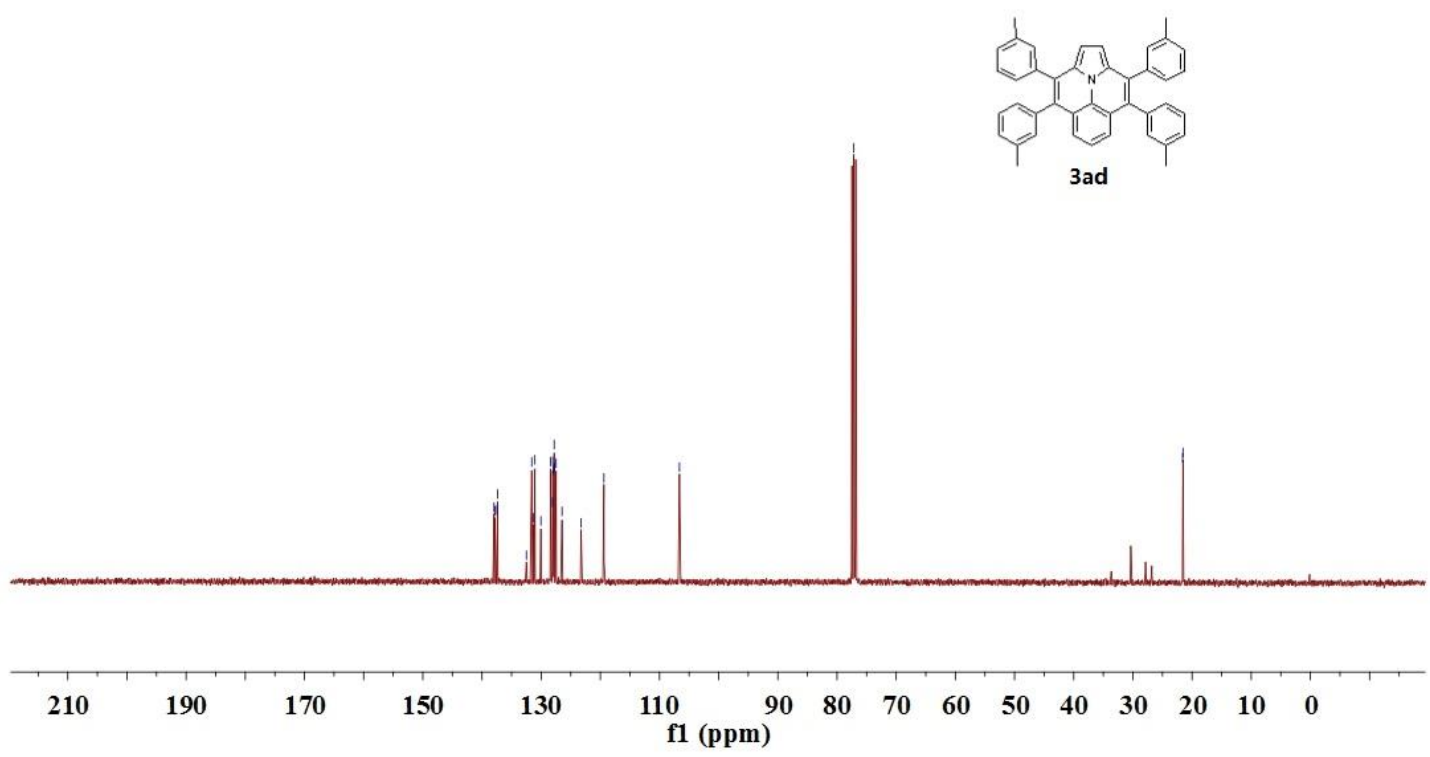



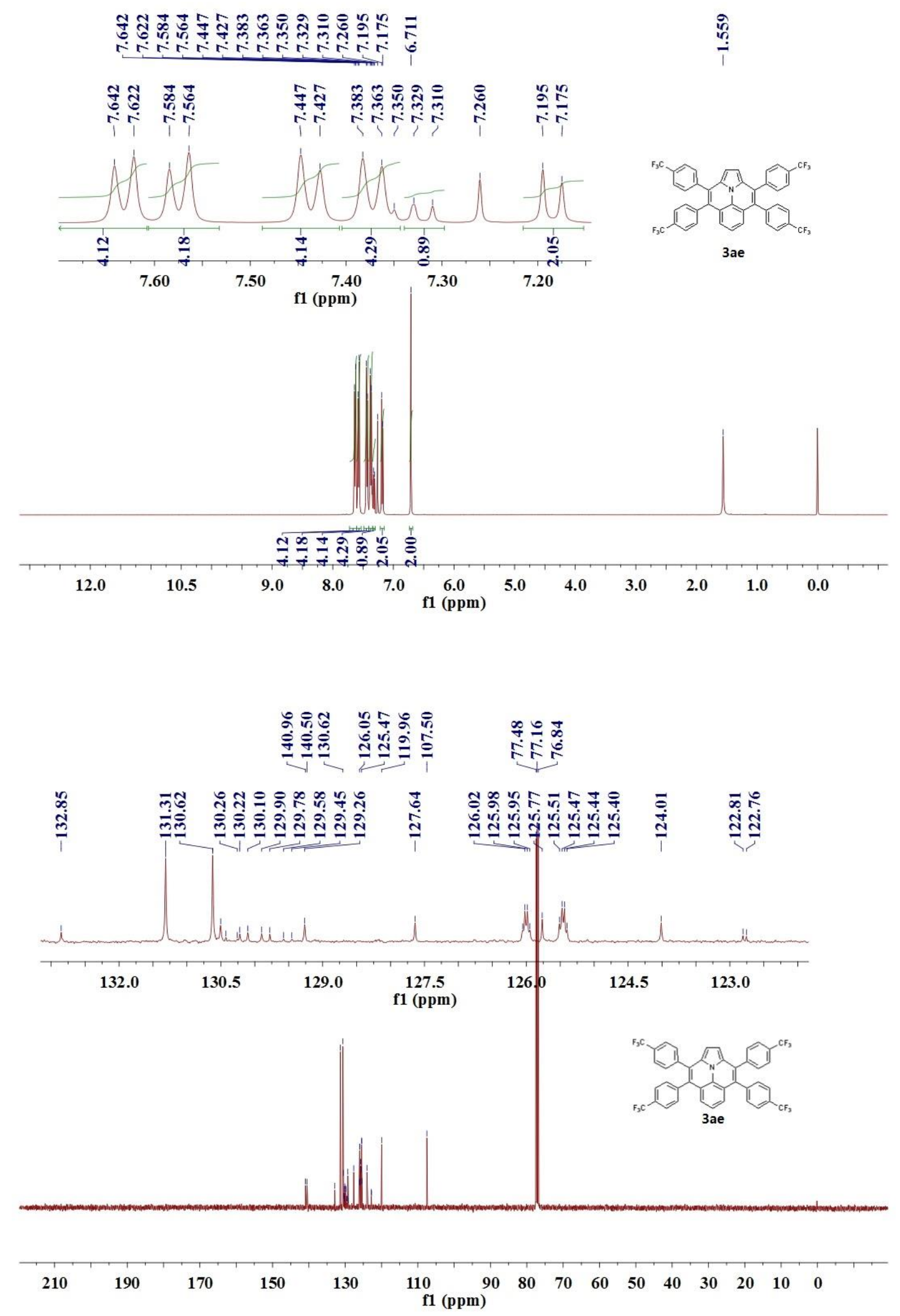


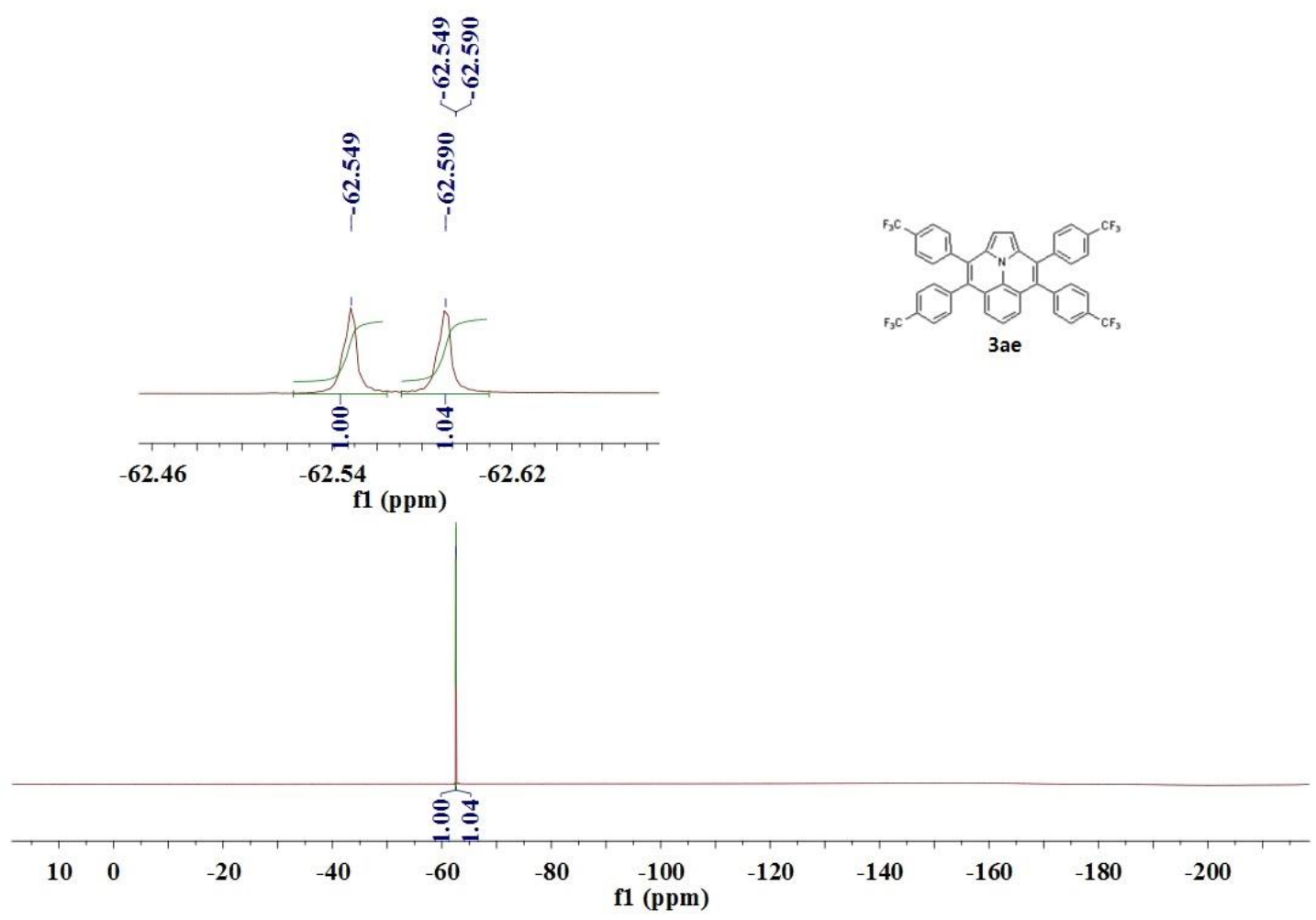

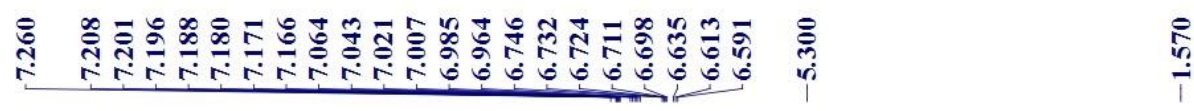

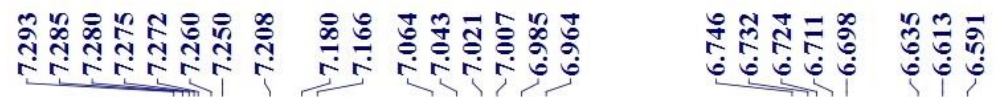
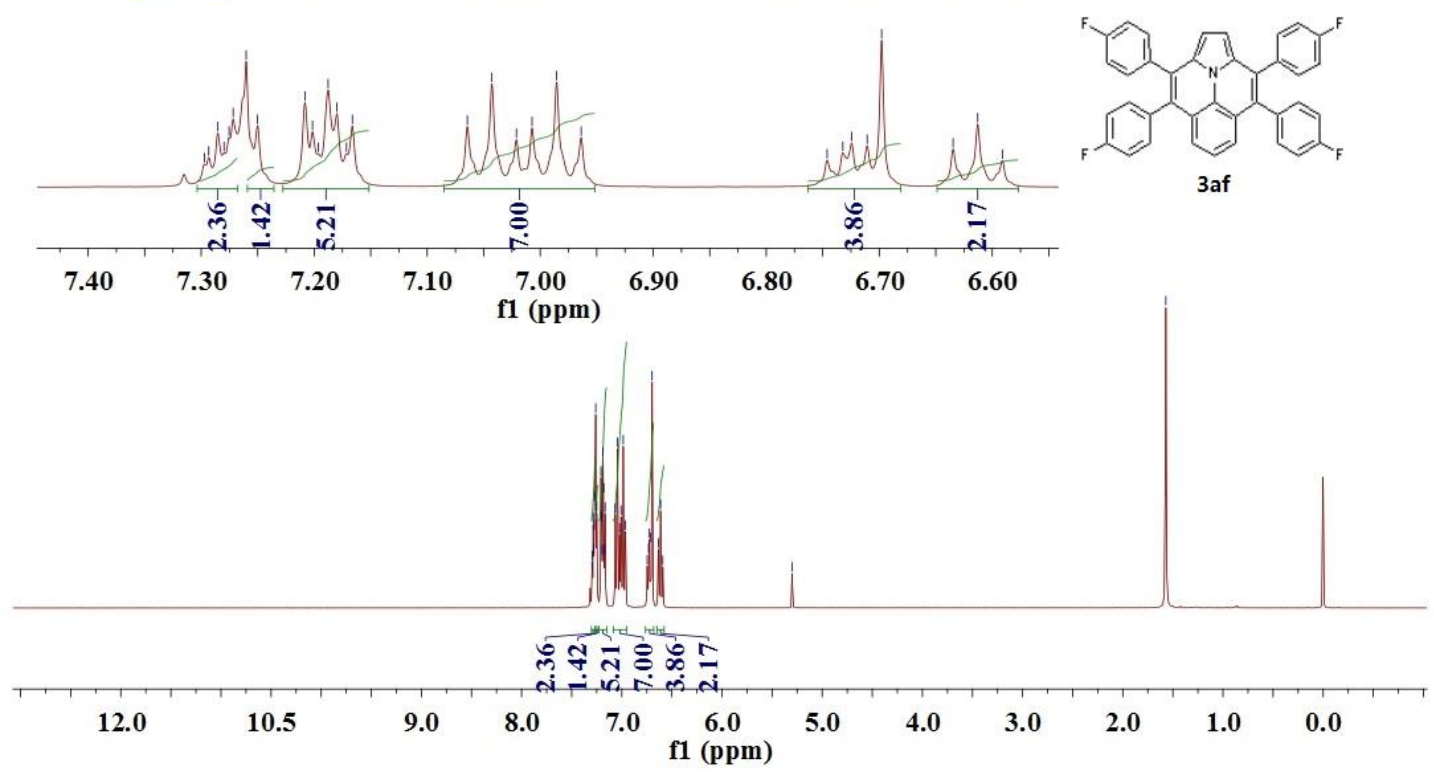


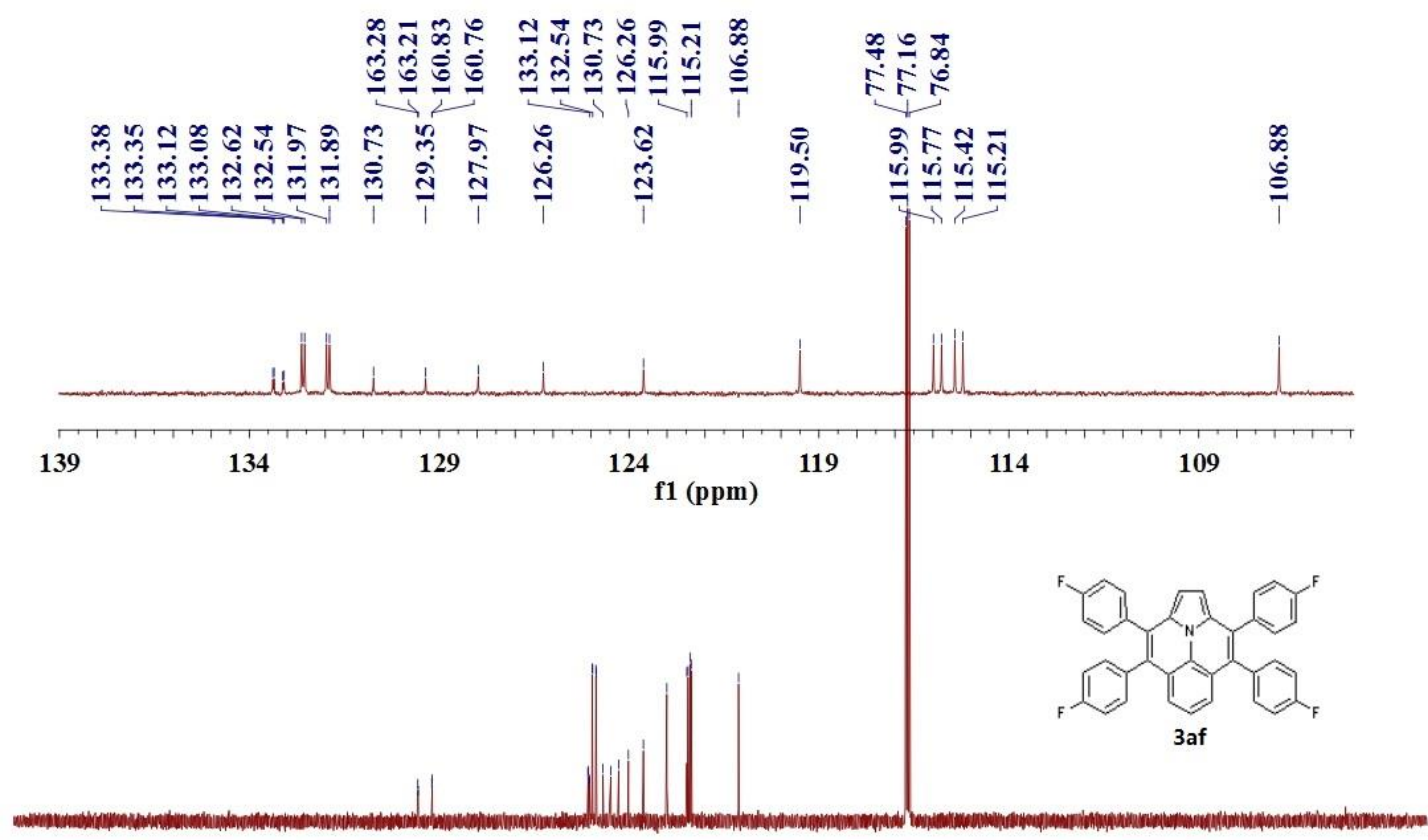

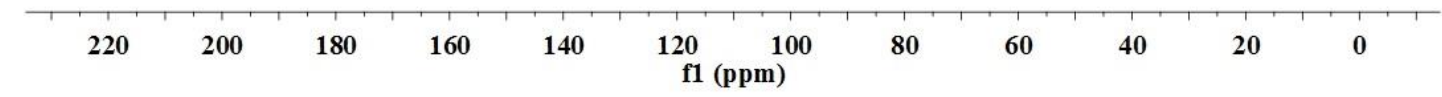

尝草草

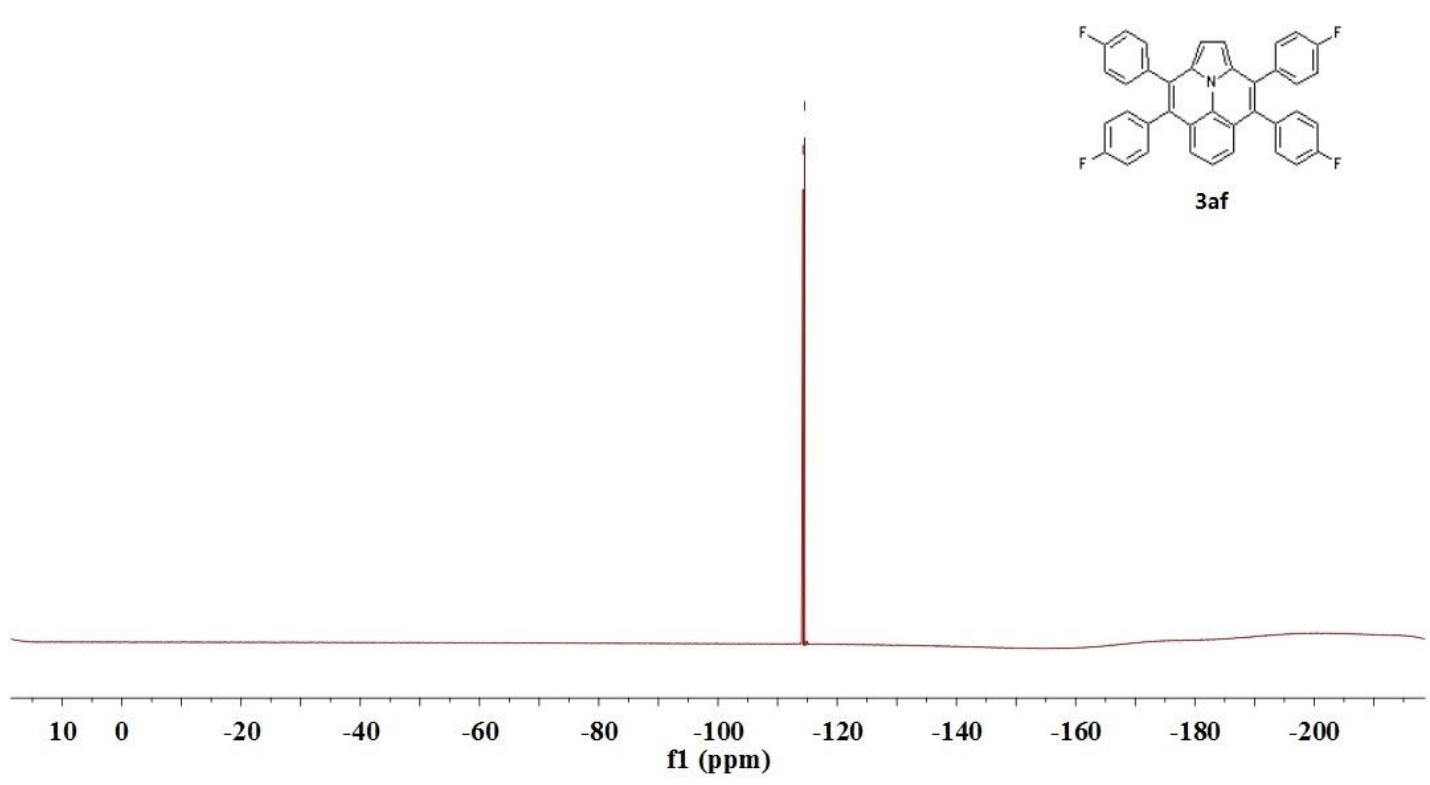




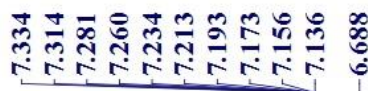

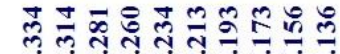

की
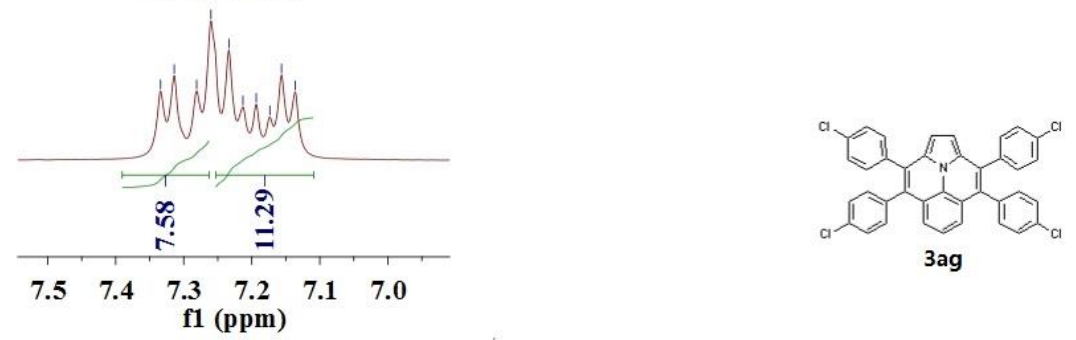

3ag

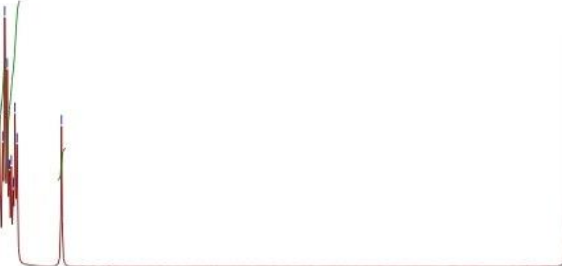

$\stackrel{\text { กे }}{=}$

$\begin{array}{lllllllllll}12.0 & 10.5 & 9.0 & 8.0 & 7.0 \underset{\mathrm{f} 1 \underset{(\mathrm{ppm})}{6.0}}{6.0} & 5.0 & 4.0 & 3.0 & 2.0 & 1.0 & 0.0\end{array}$

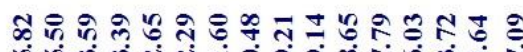

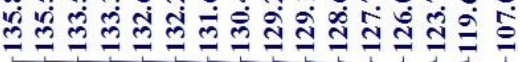
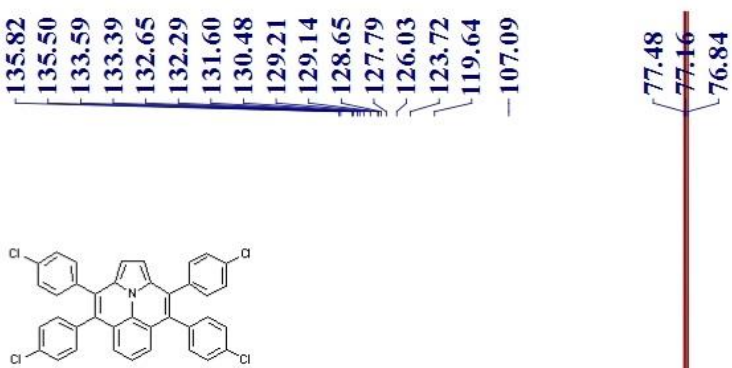

3ag
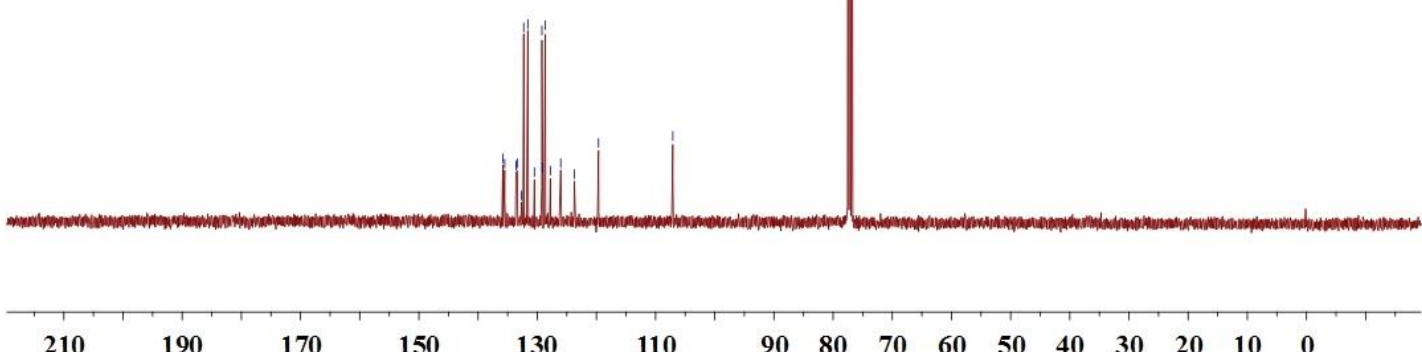

150

130

$110 \quad 90$

f1 (ppm) 


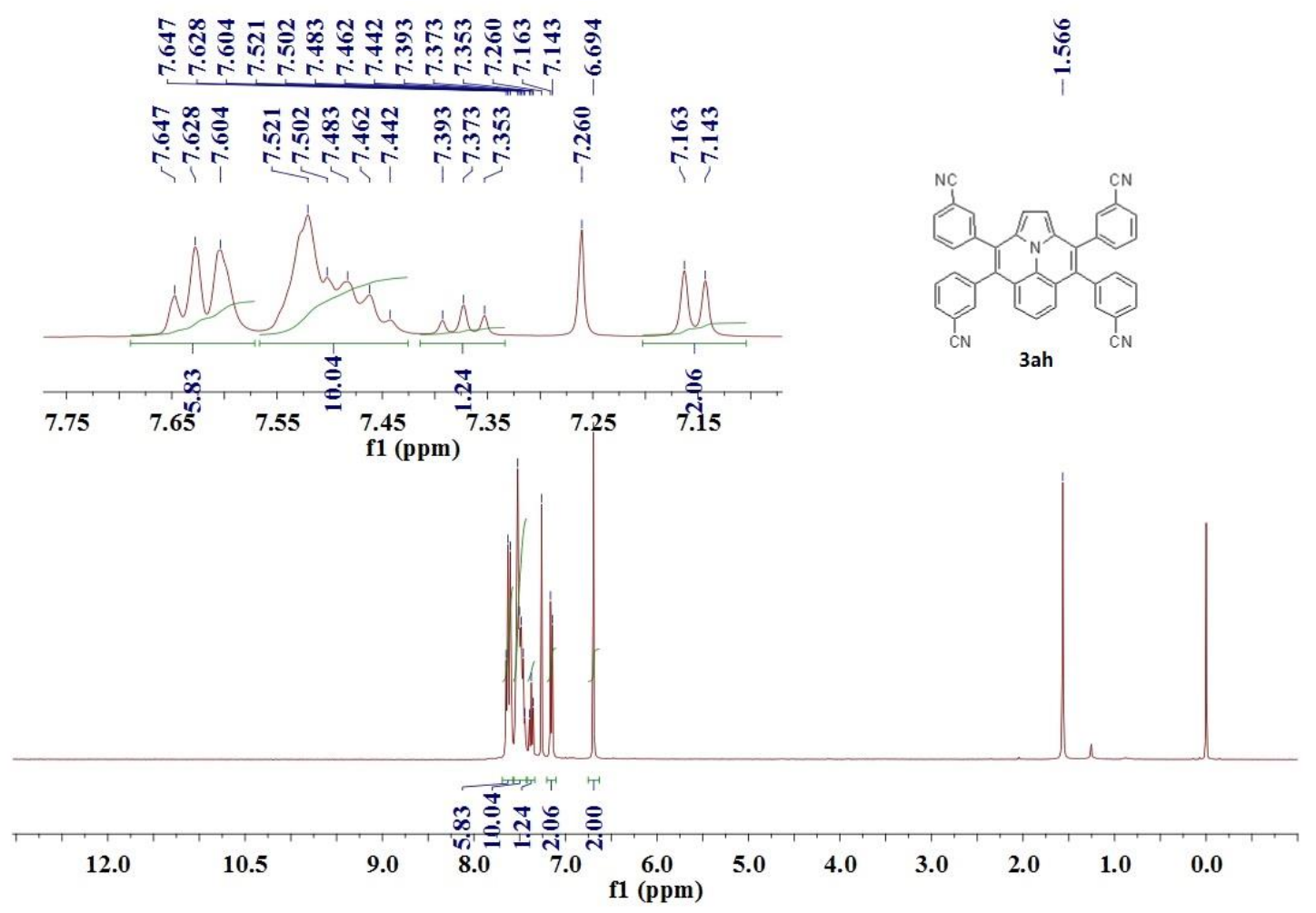

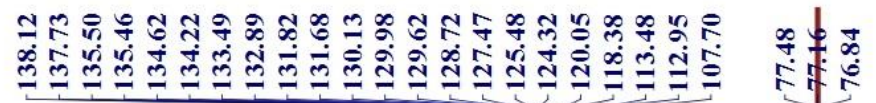

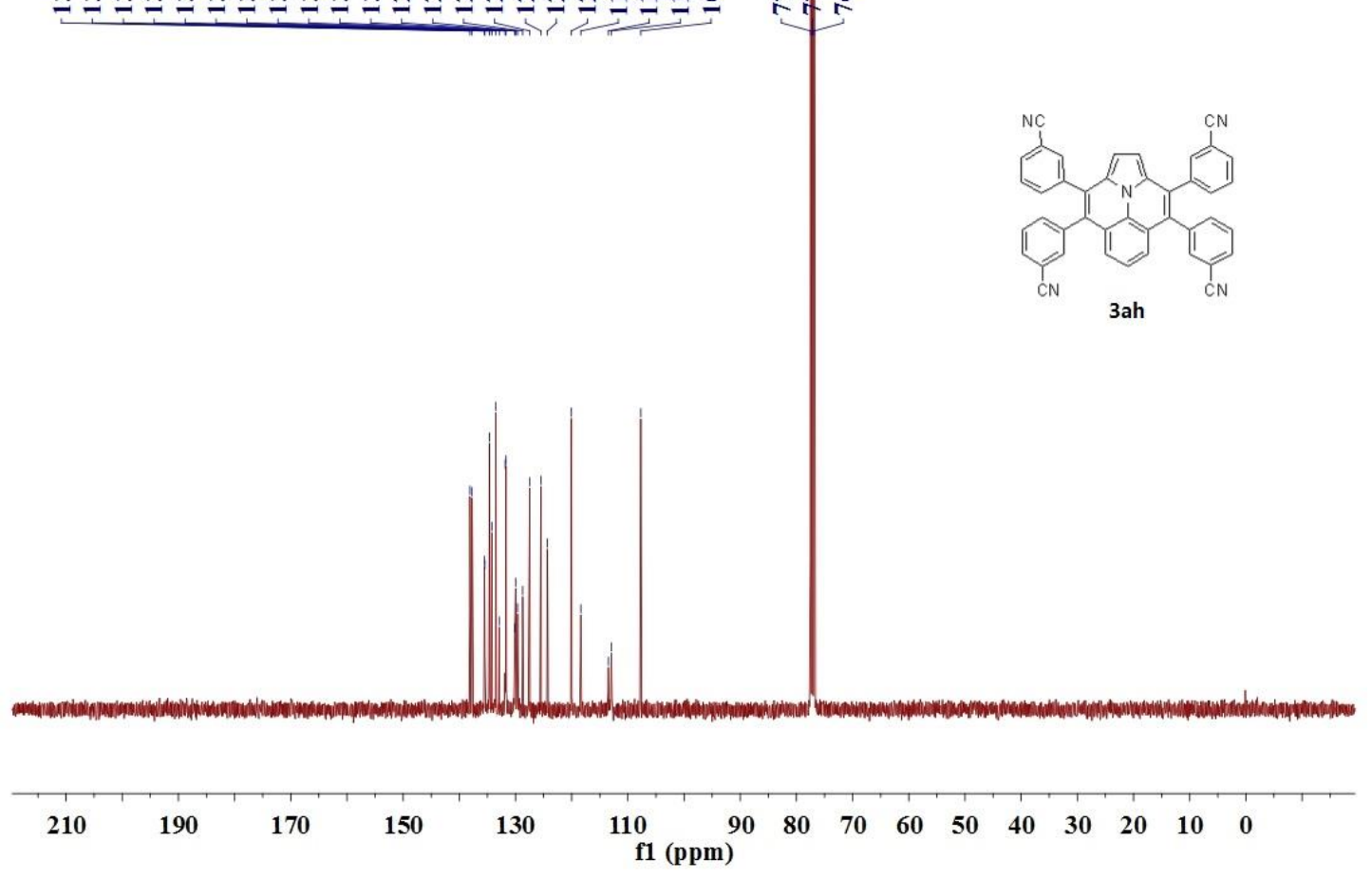




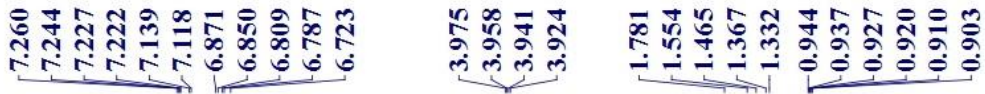

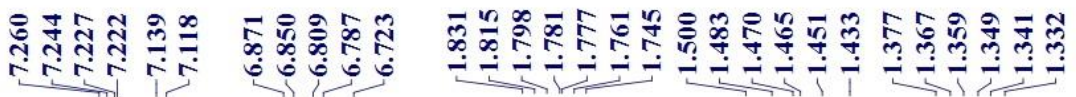
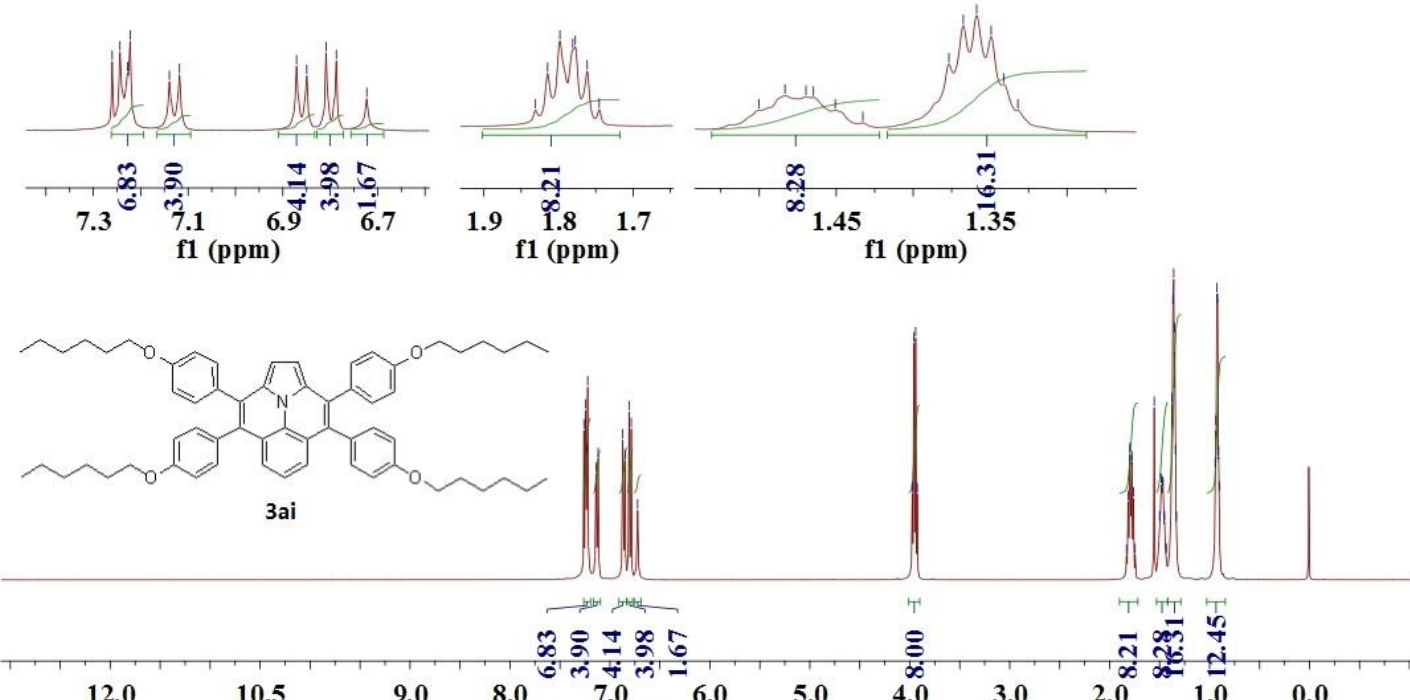

12.0
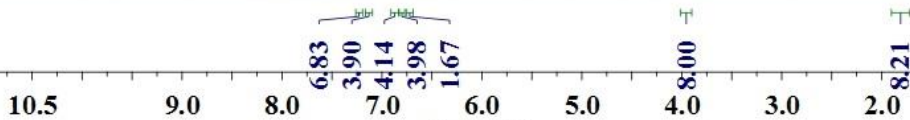

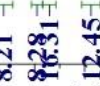
f1 (ppm)

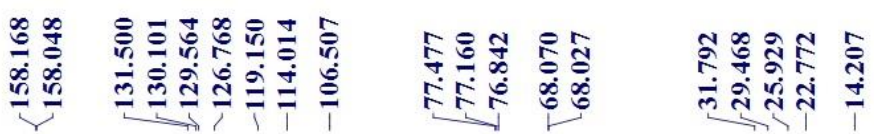

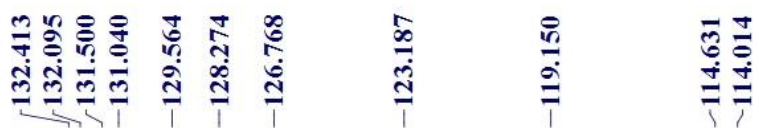
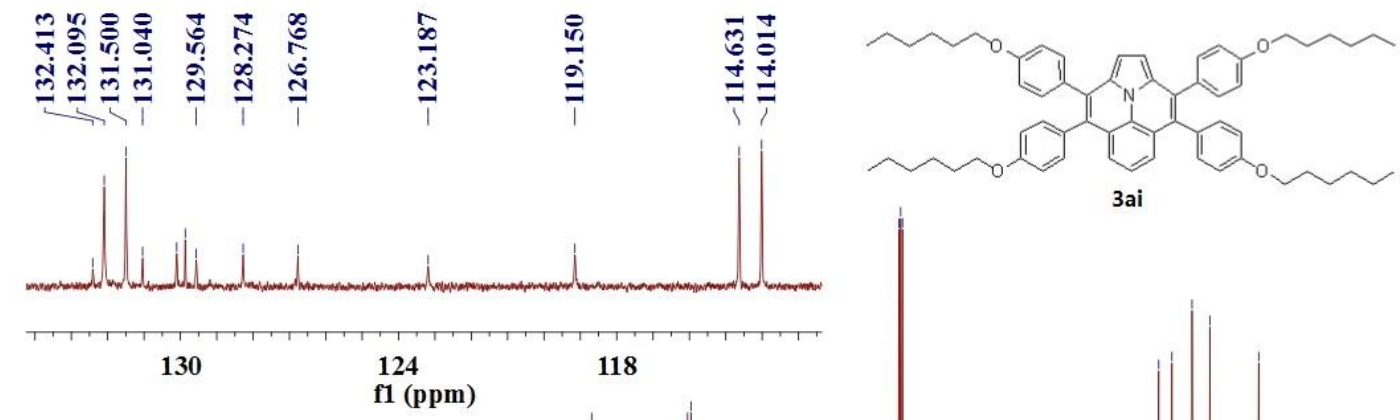

3ai

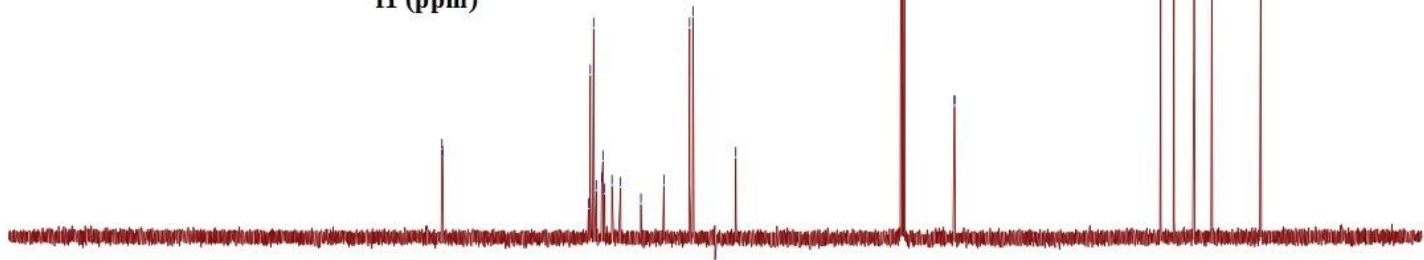

220

200

180

120

f1 (ppm) 


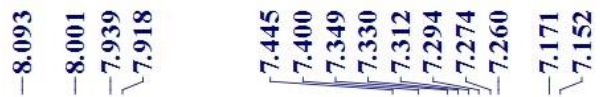

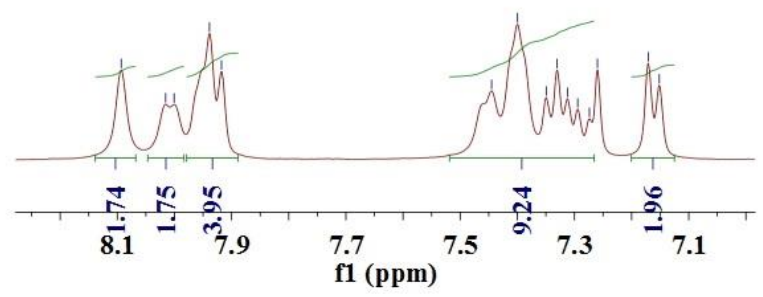

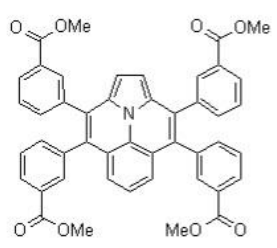

3aj

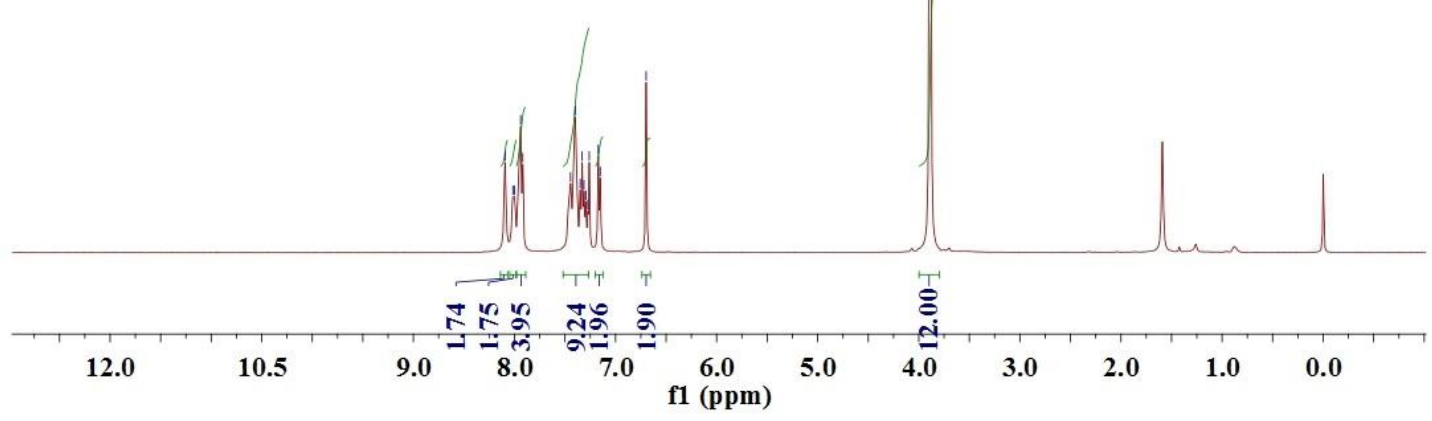

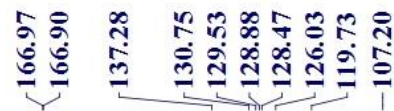

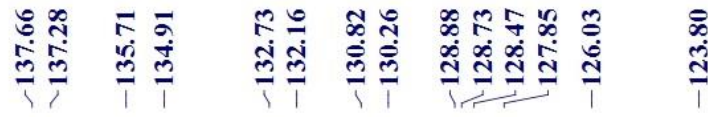

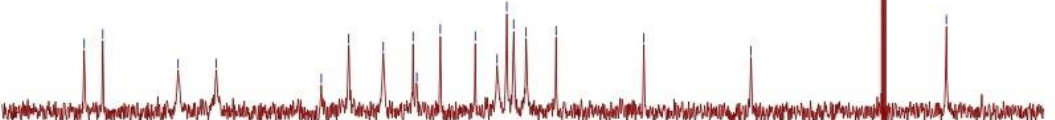

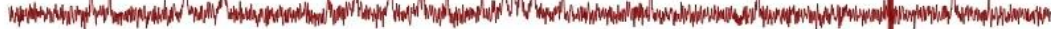

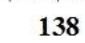

135

132

129

126

123

120

$\stackrel{9}{1}$

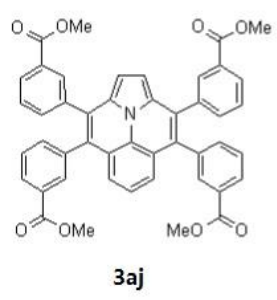

f1 (ppm)

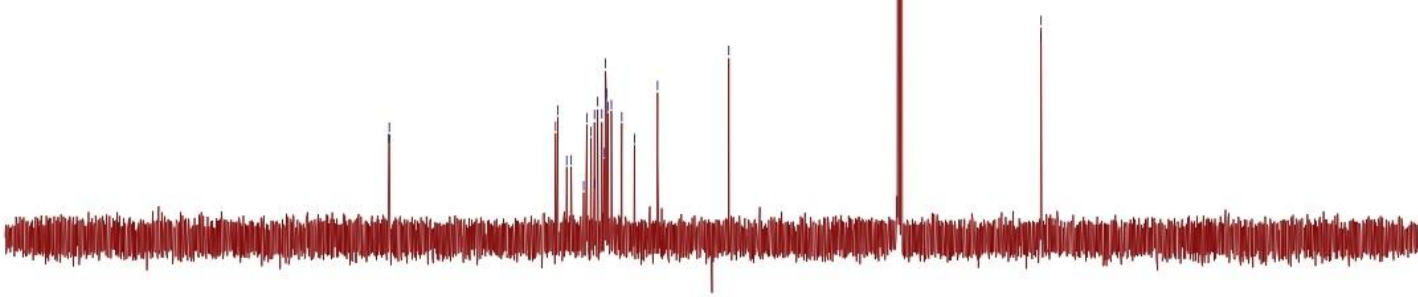

220

200

180

160

140

120 

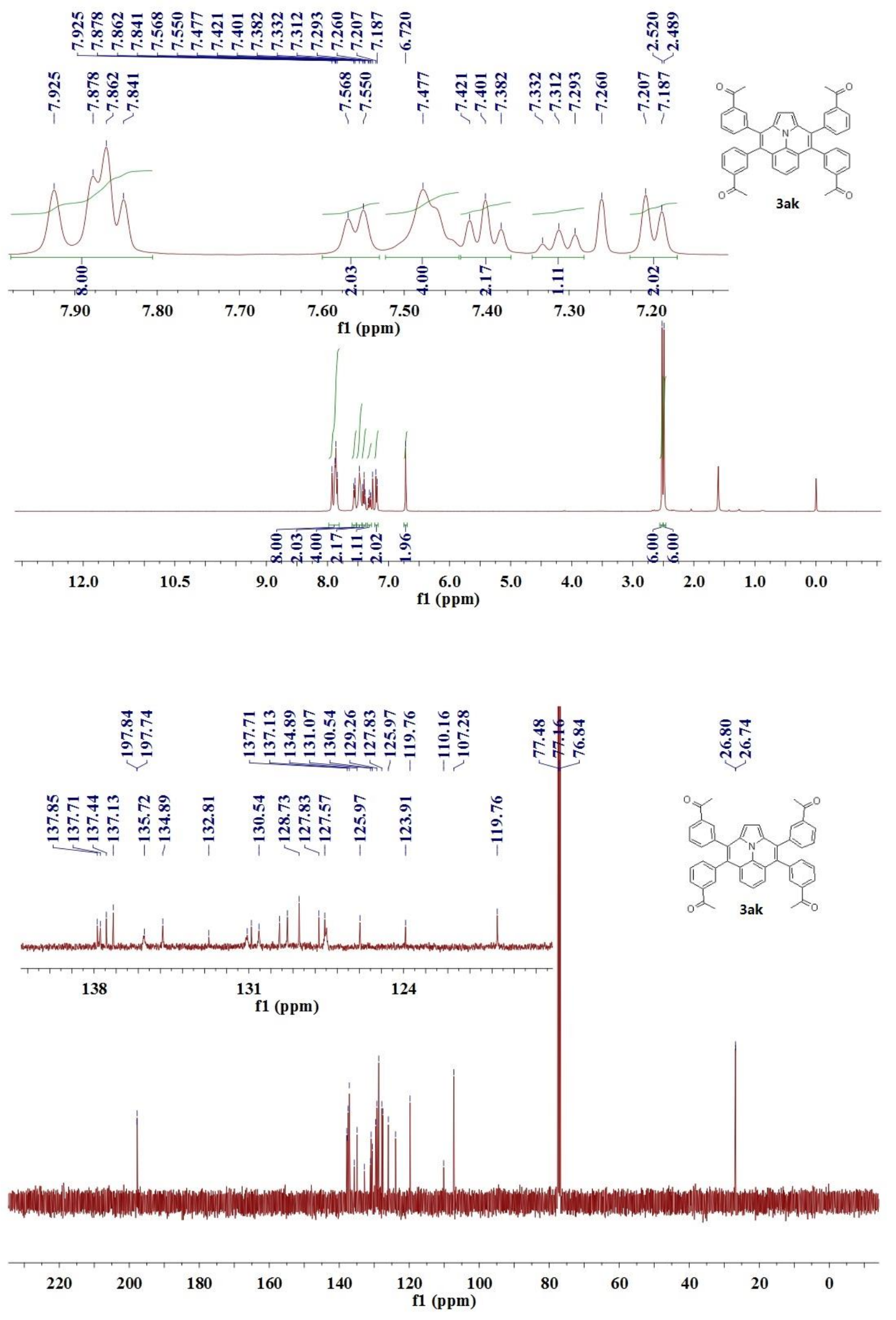

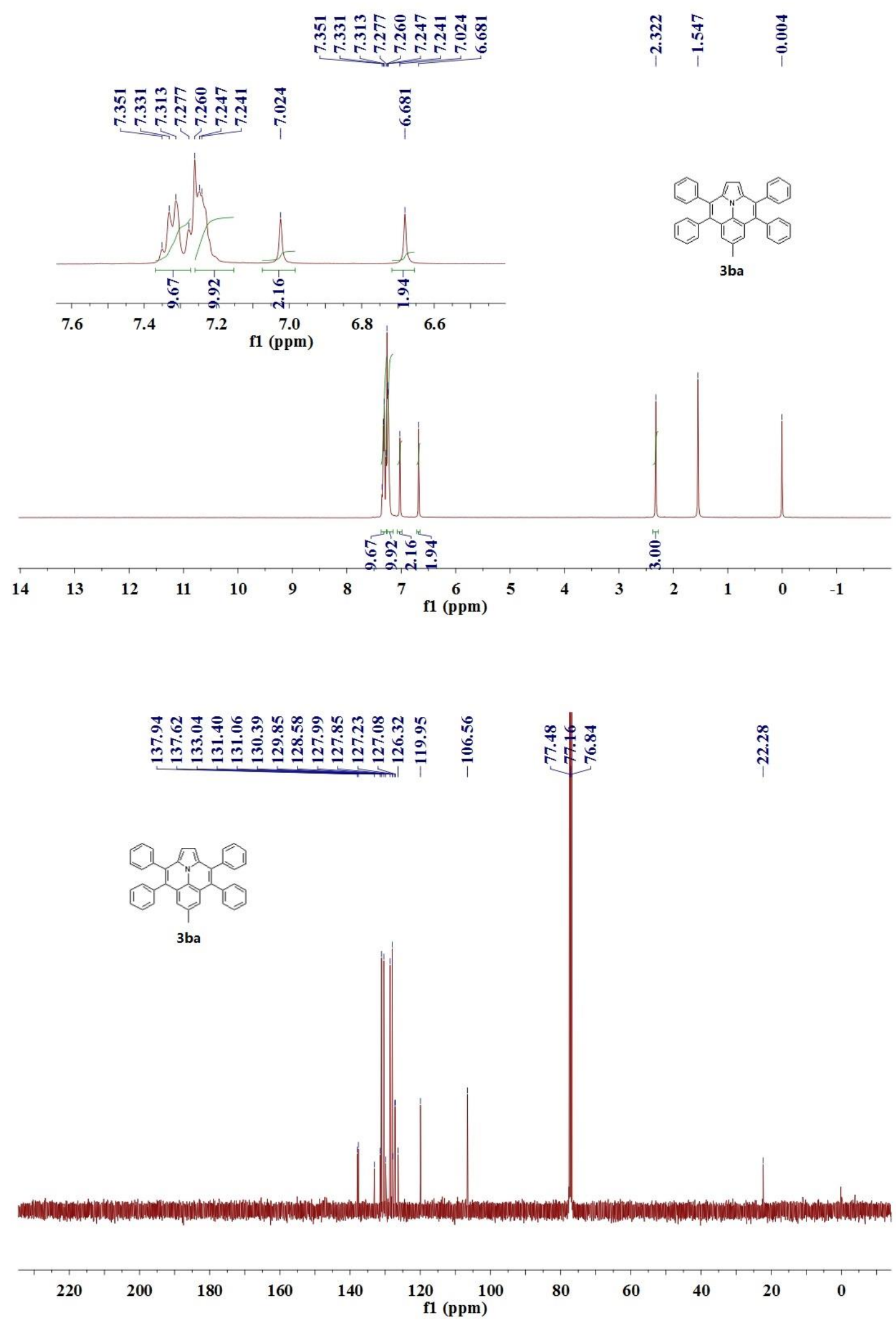

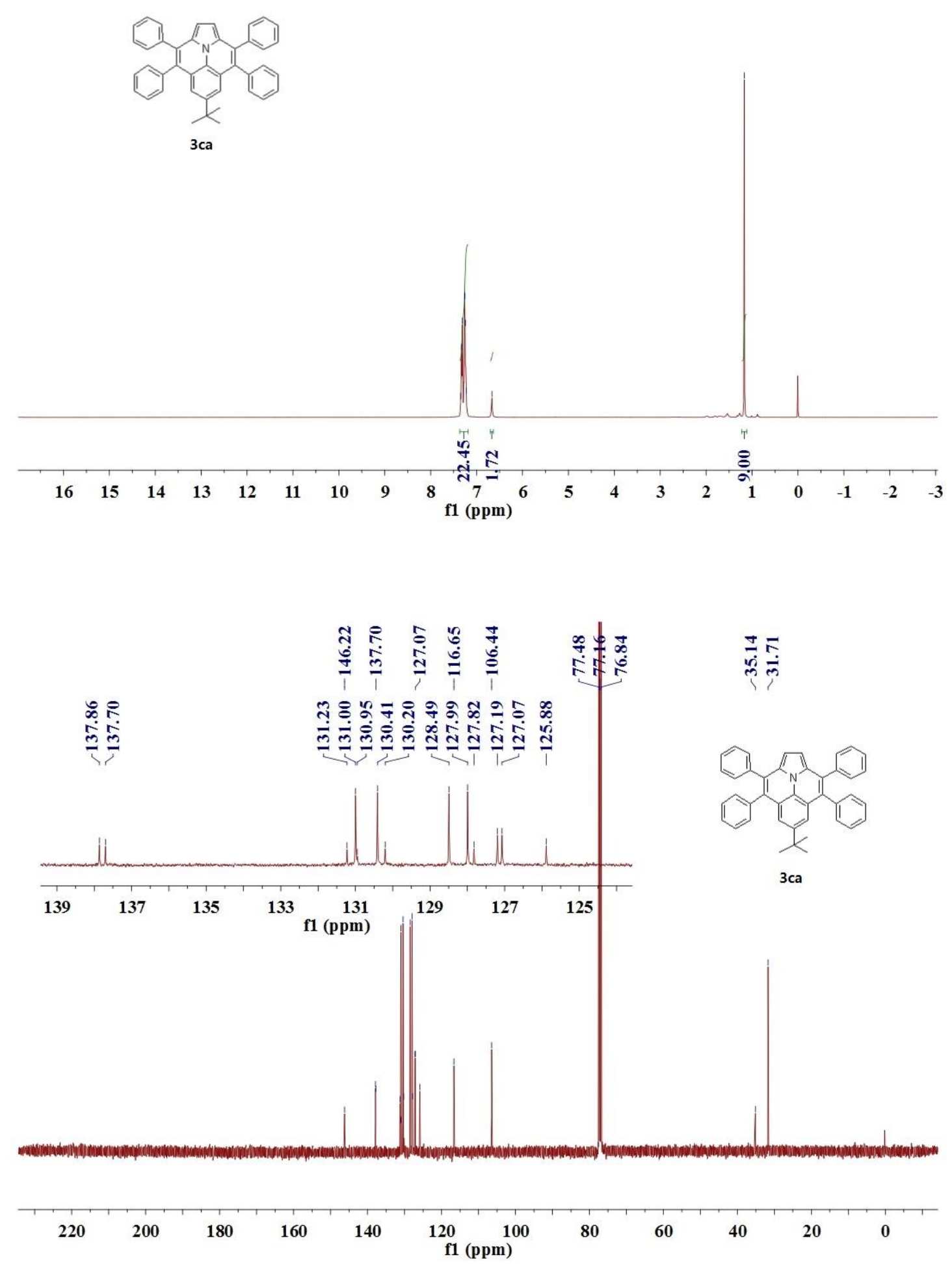


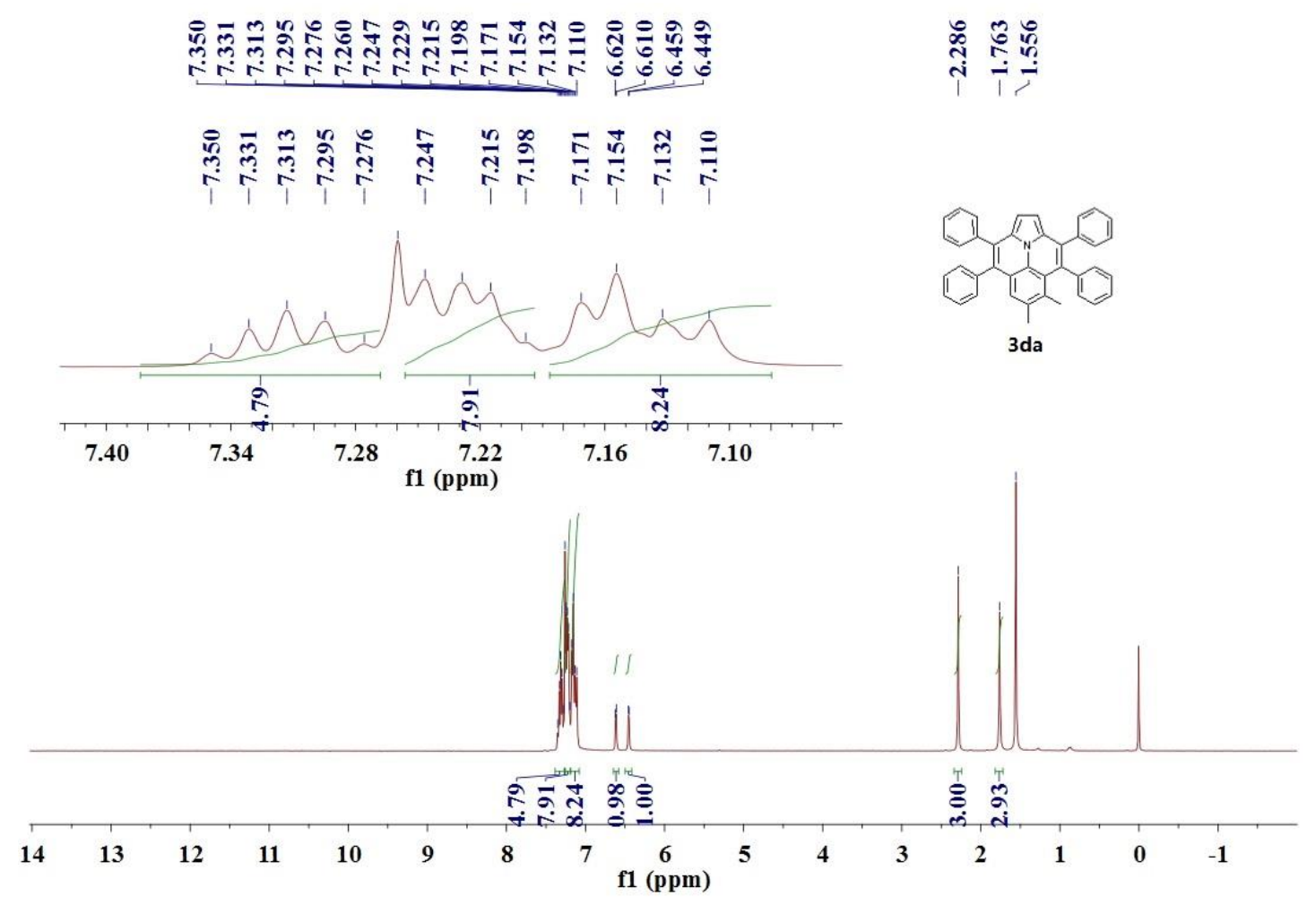

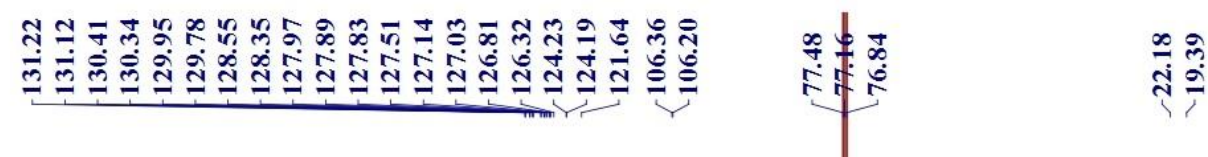

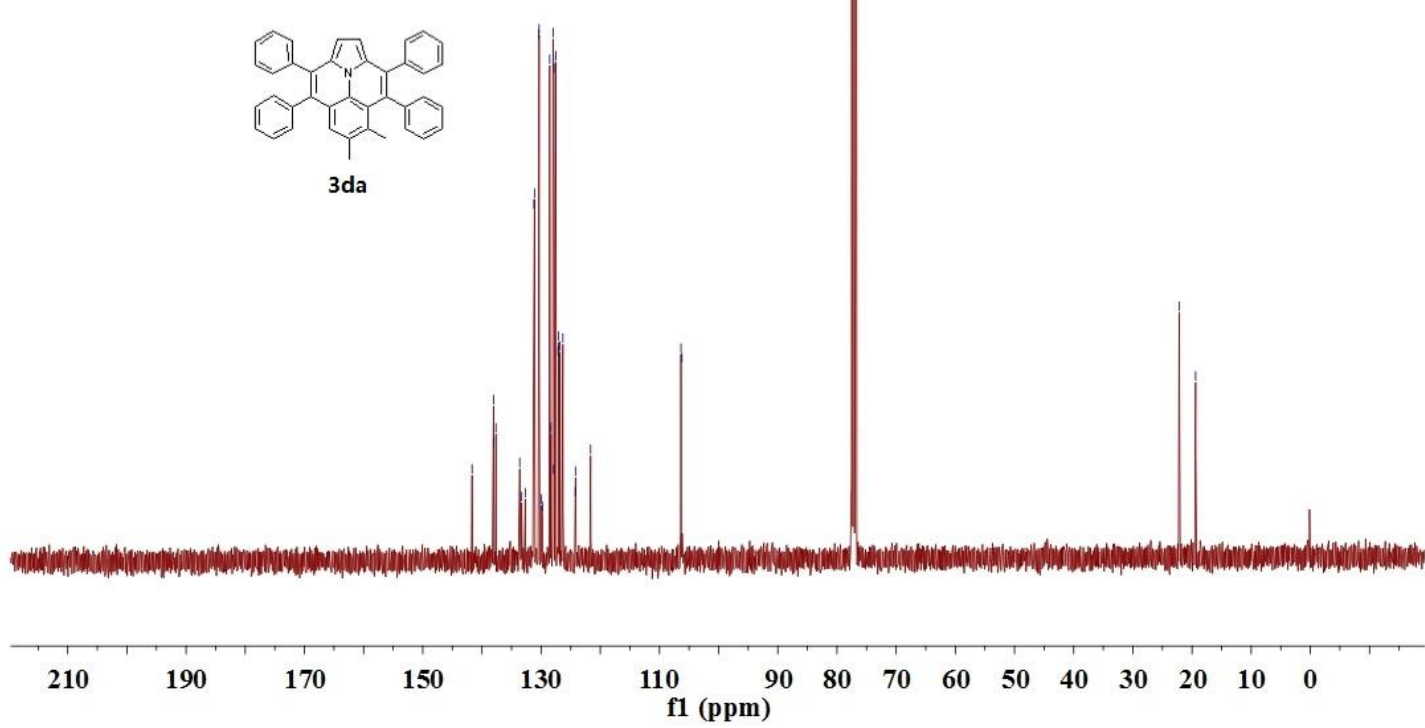




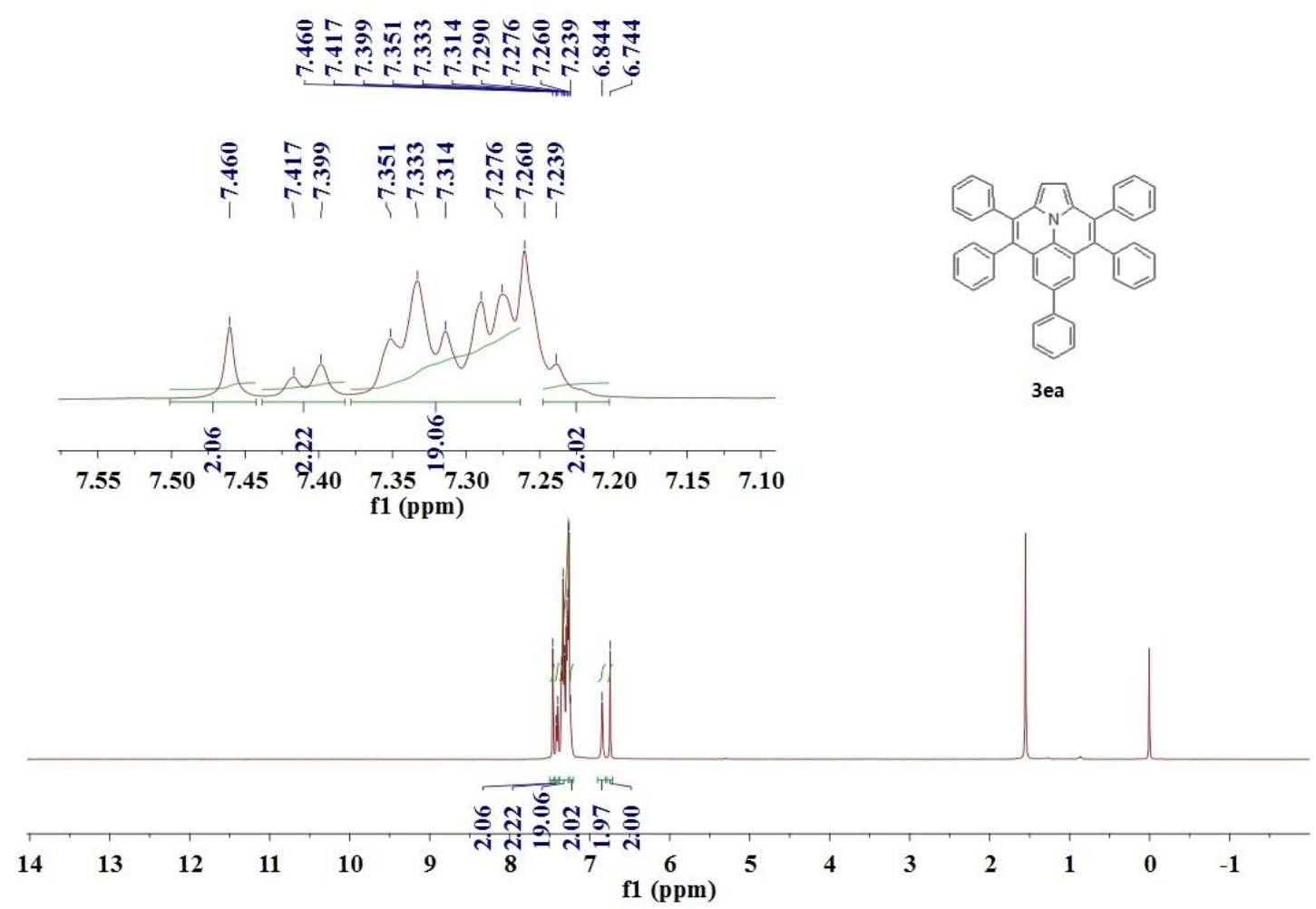

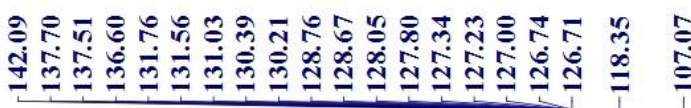

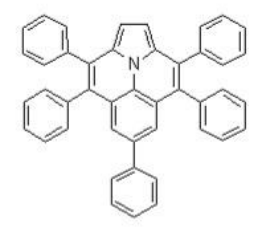

3ea
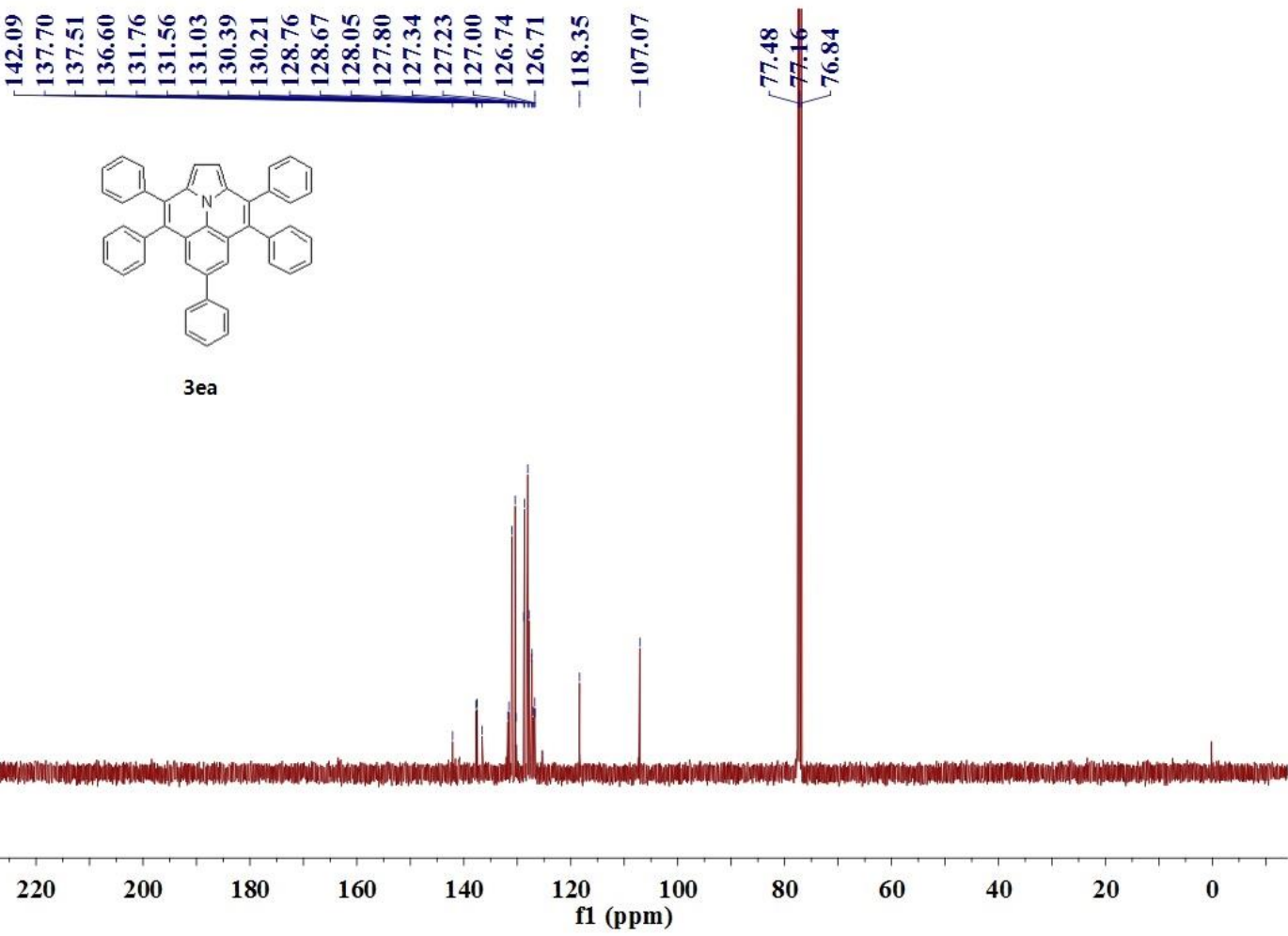

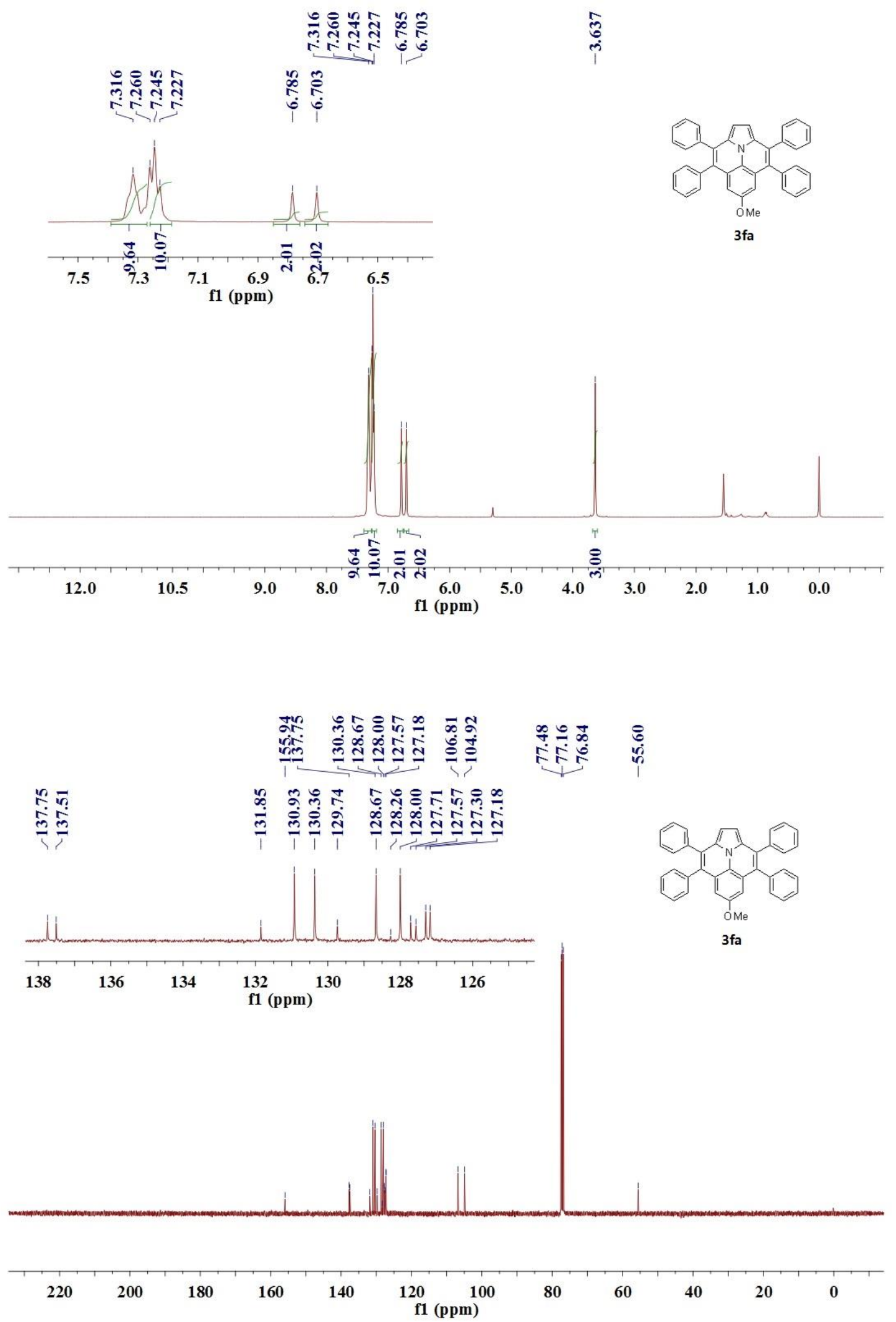


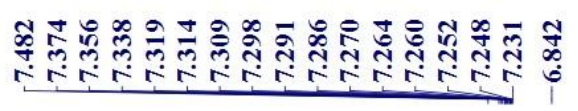

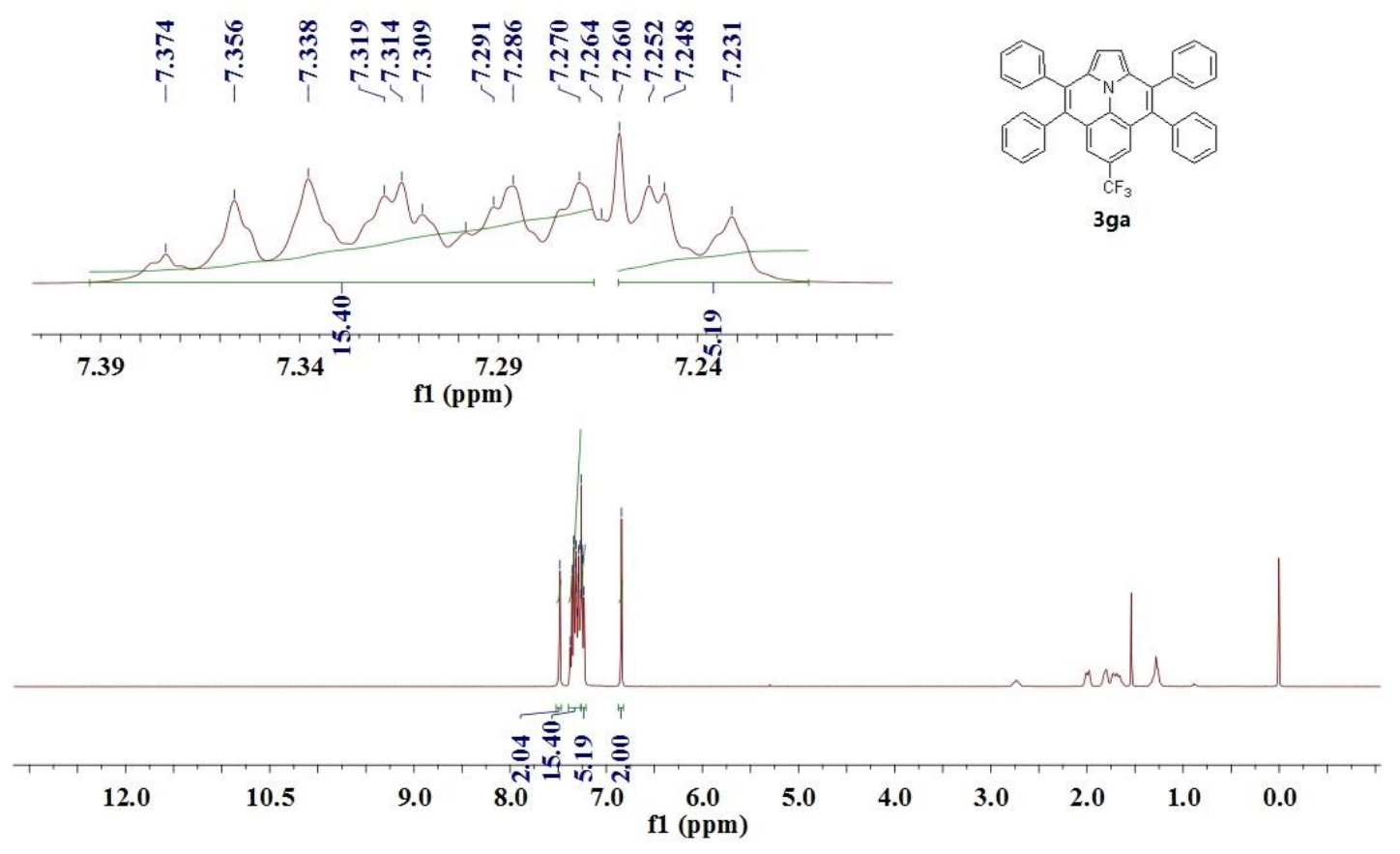

ถู

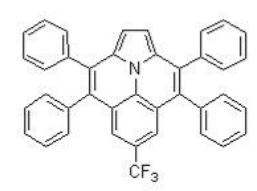

3ga

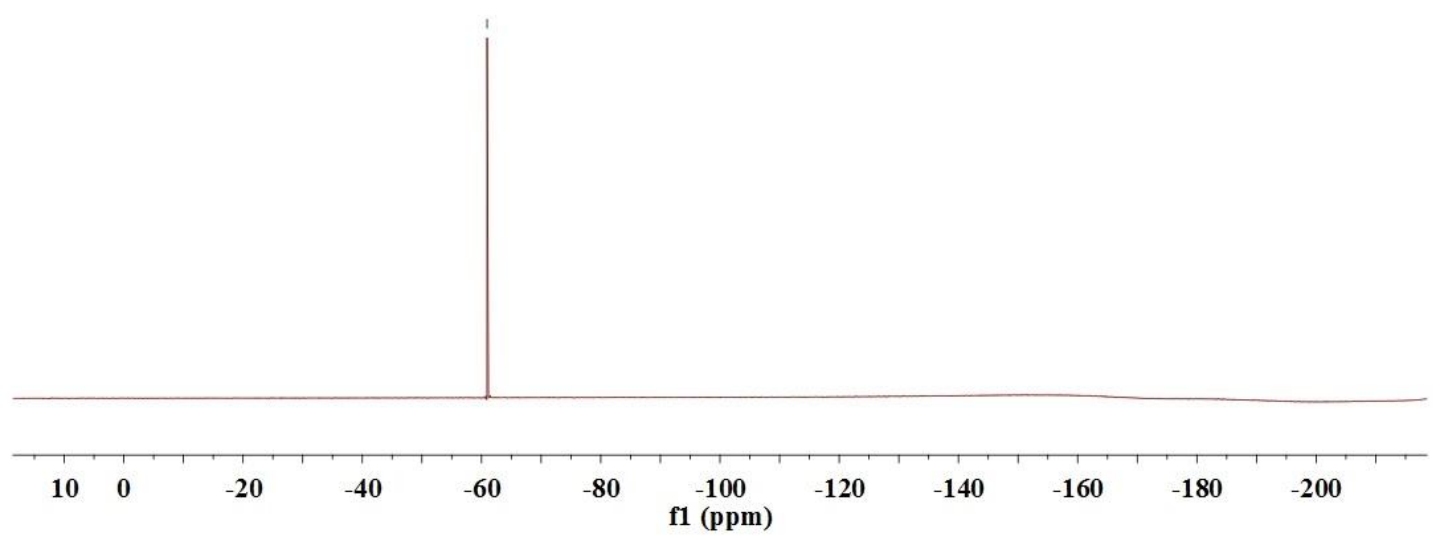



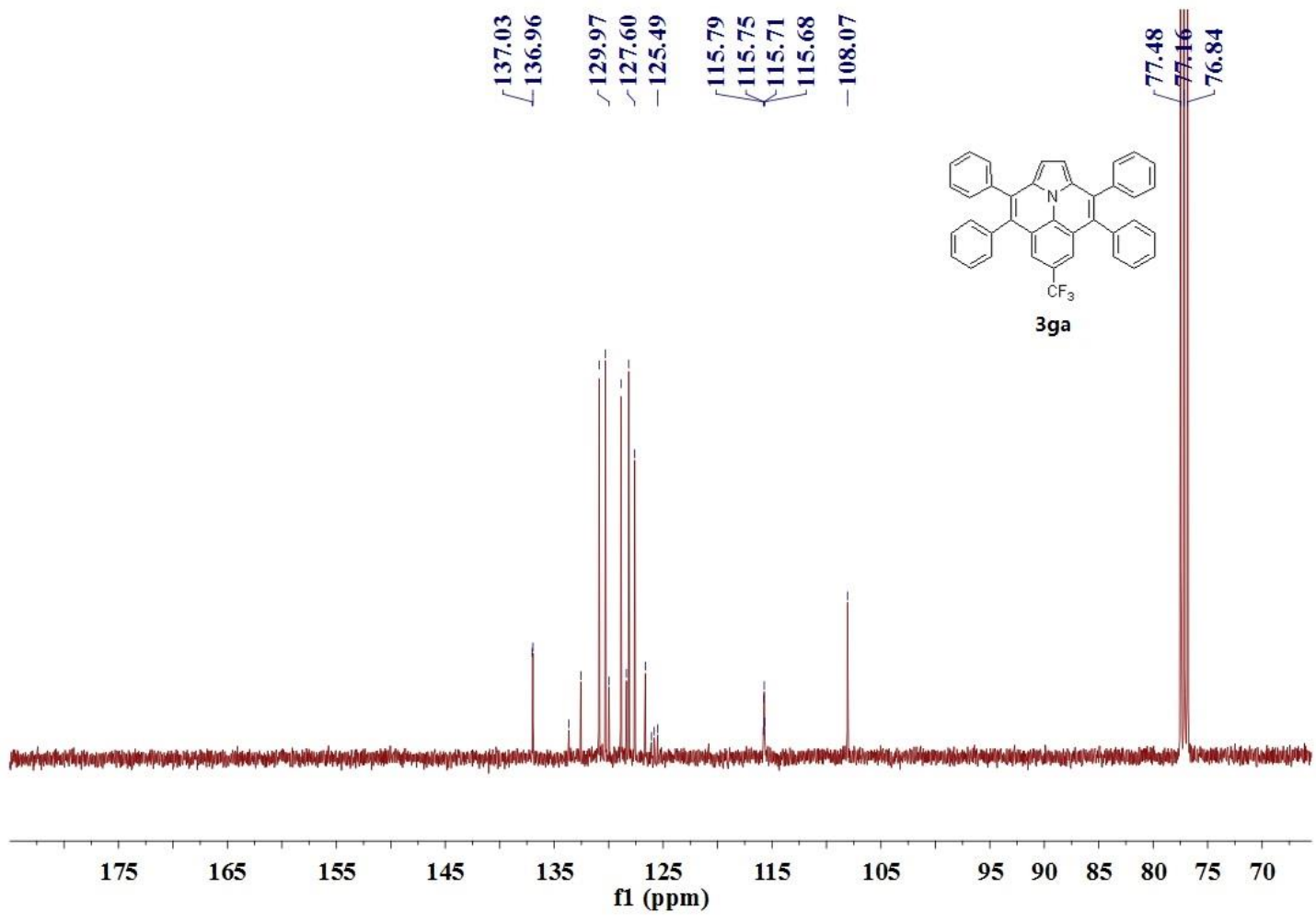

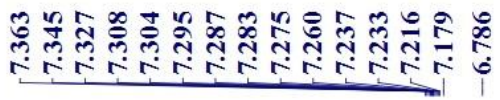

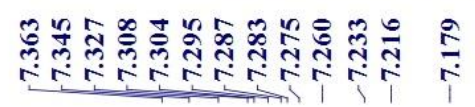
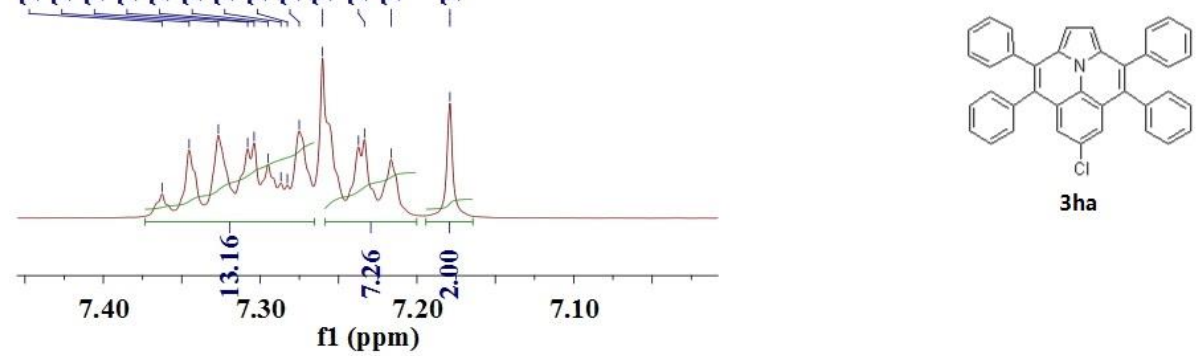

3ha

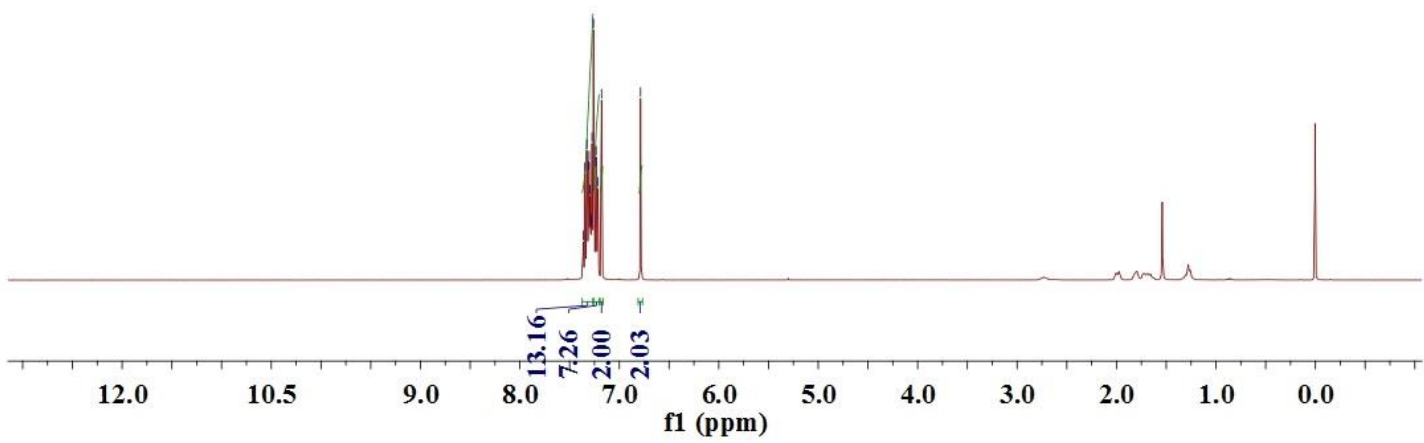




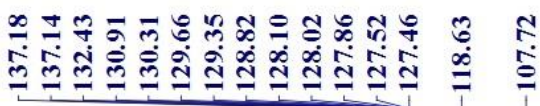

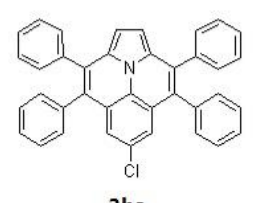

3ha

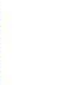

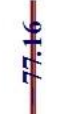

\begin{tabular}{lllllllllllllllllllll}
\hline 210 & 190 & 170 & 150 & 130 & 110 & 90 & 80 & 70 & 60 & 50 & 40 & 30 & 20 & 10 & 0 & &
\end{tabular}

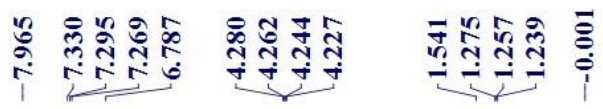

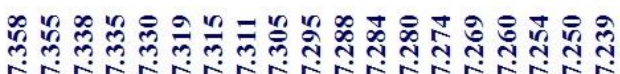

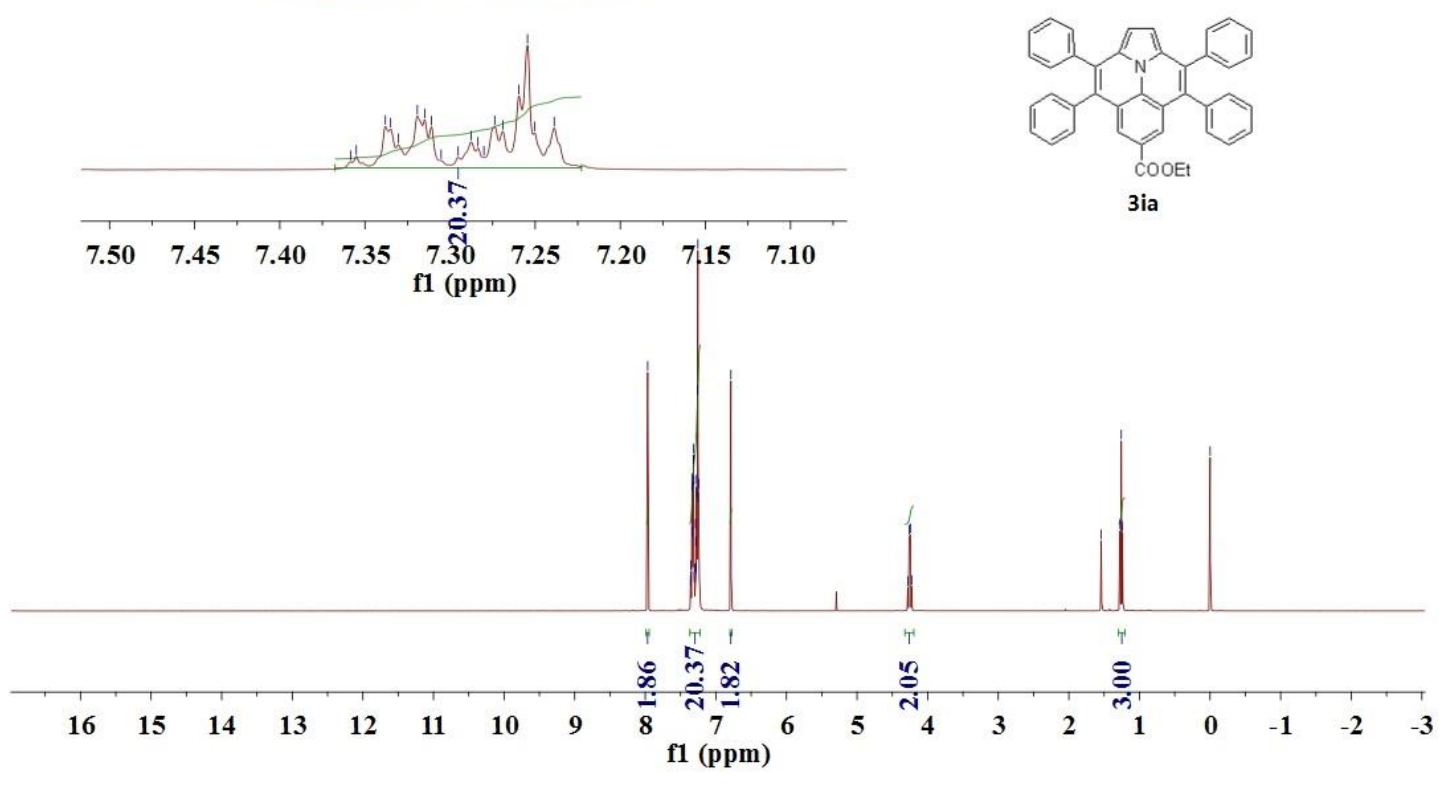




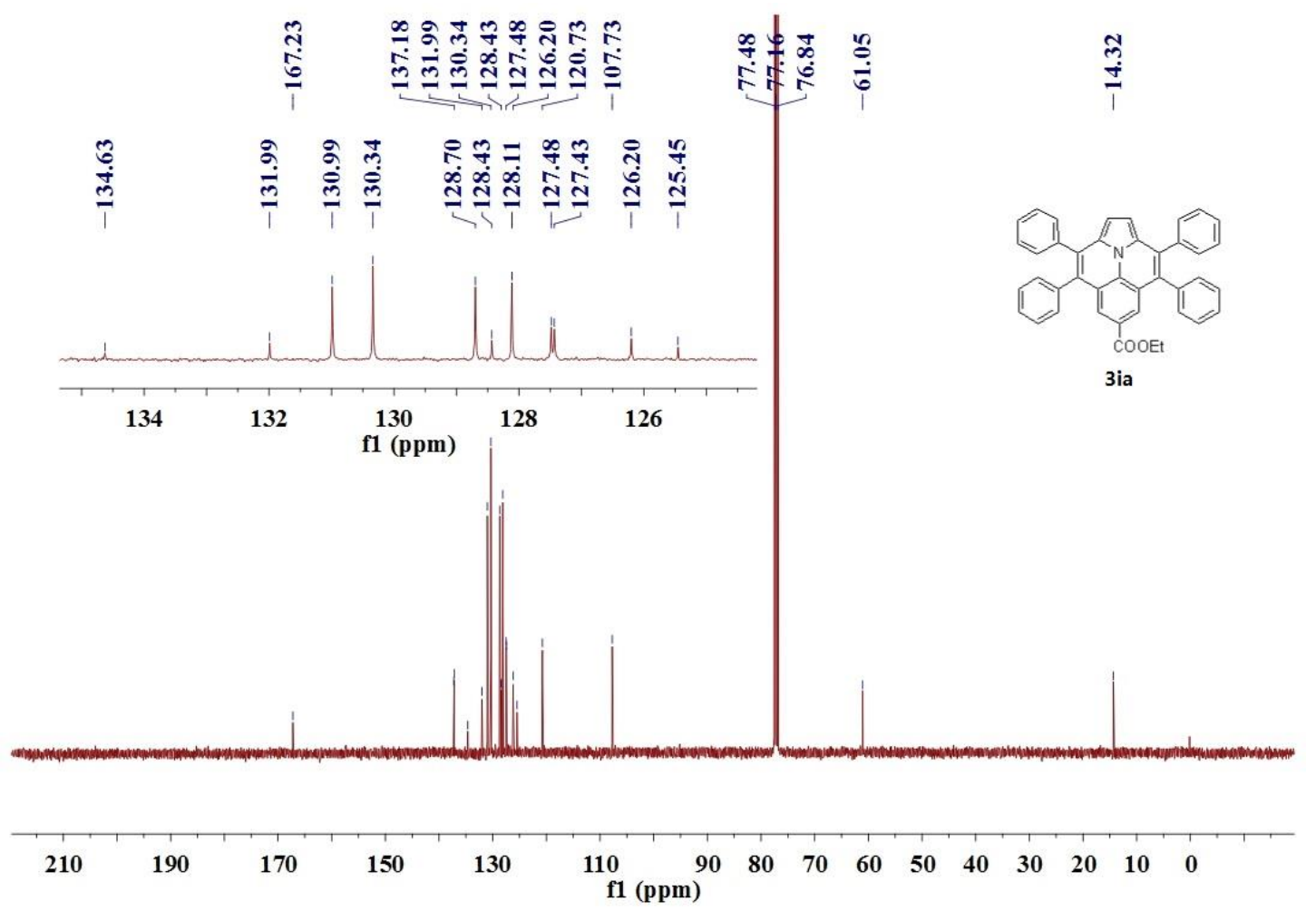

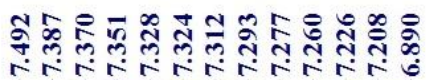

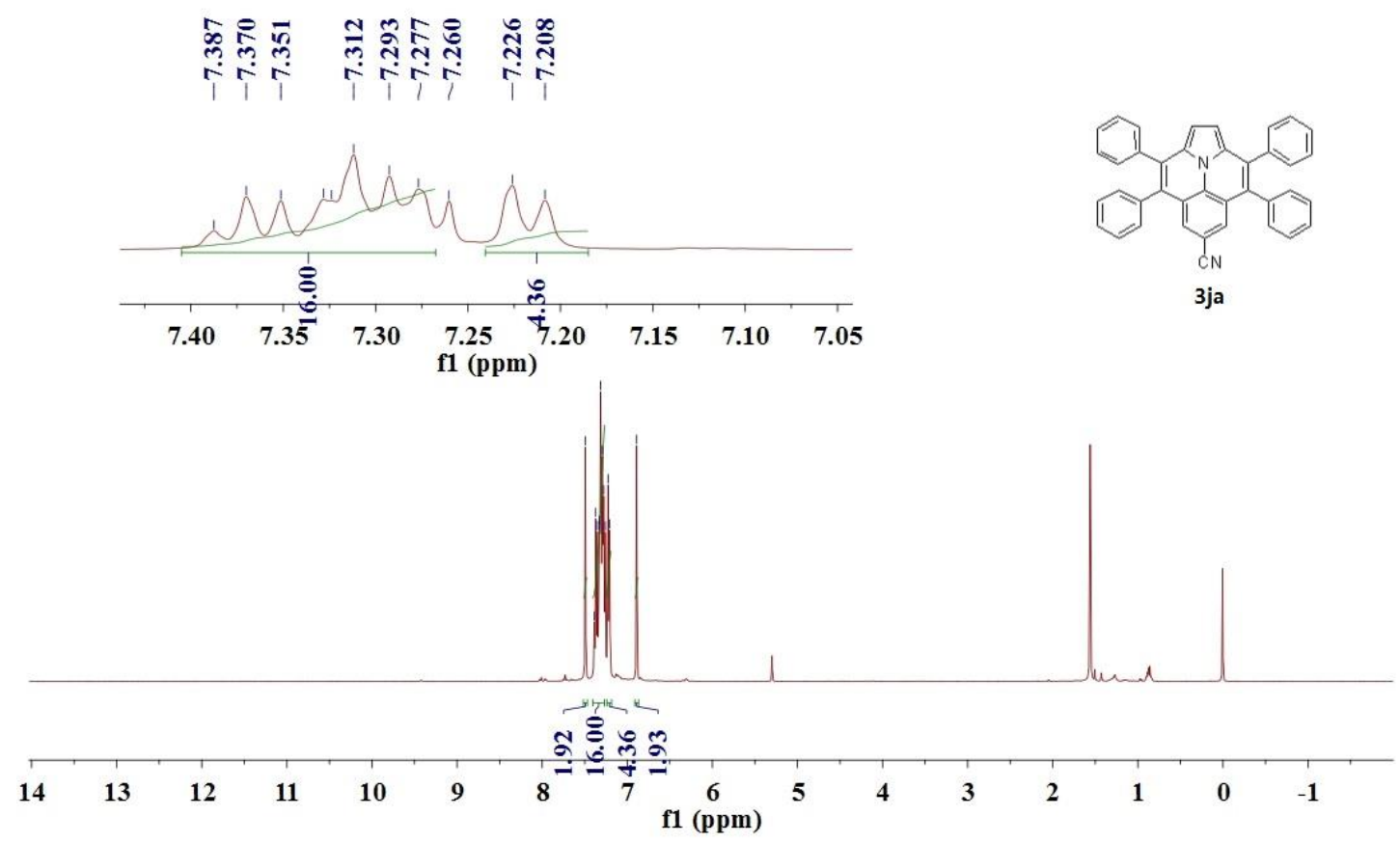




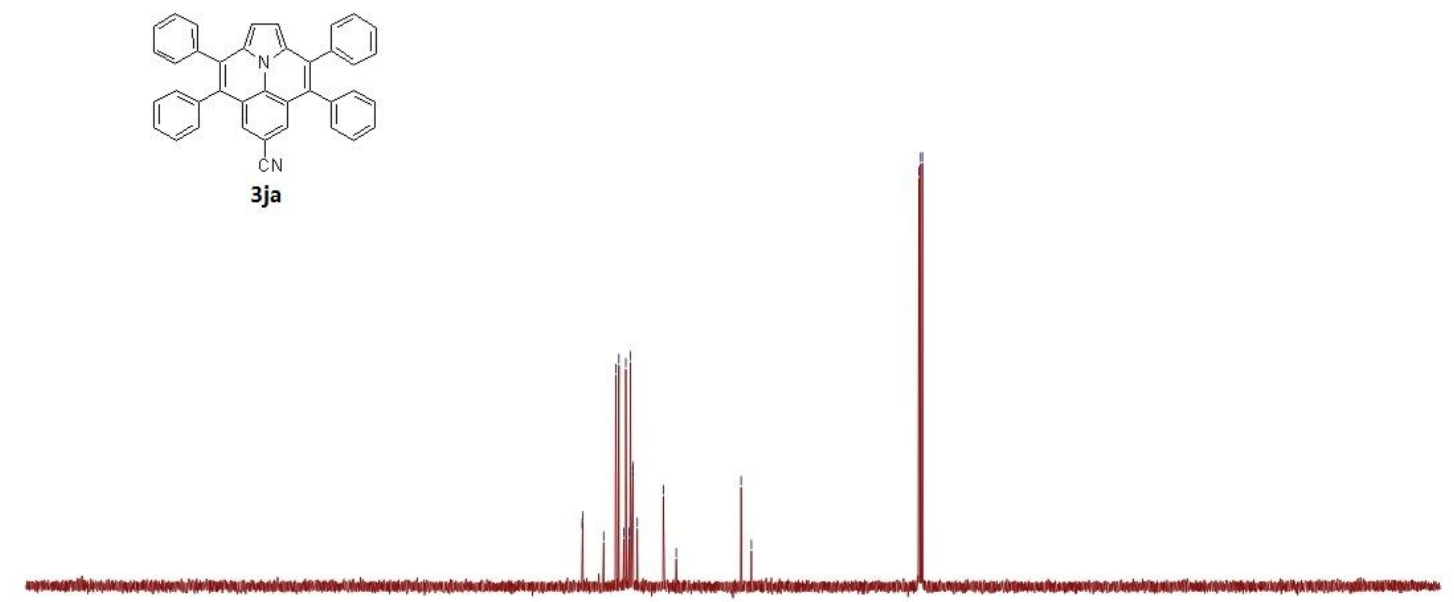

3ja

in

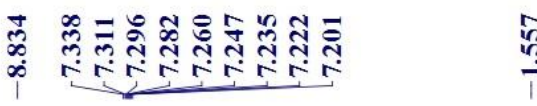

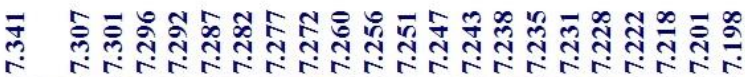

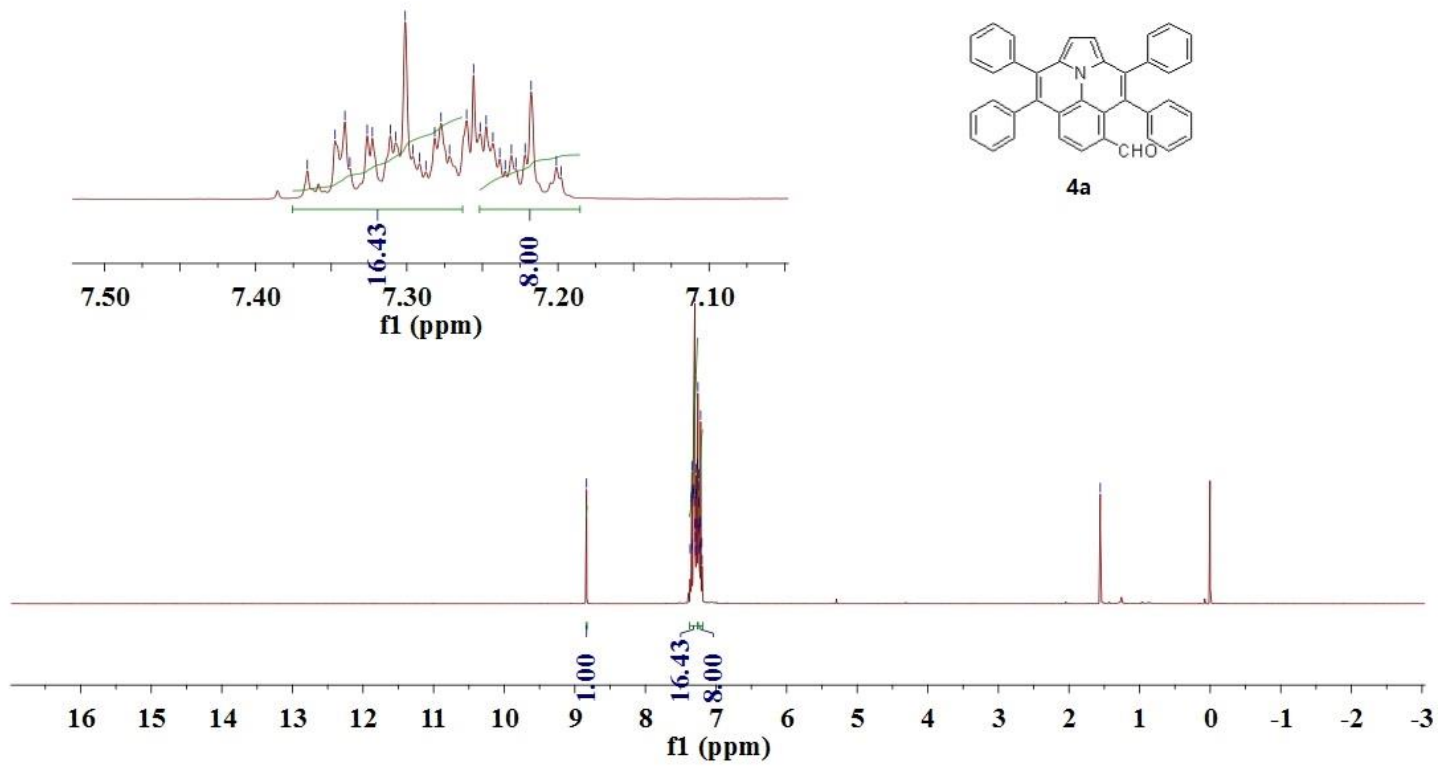




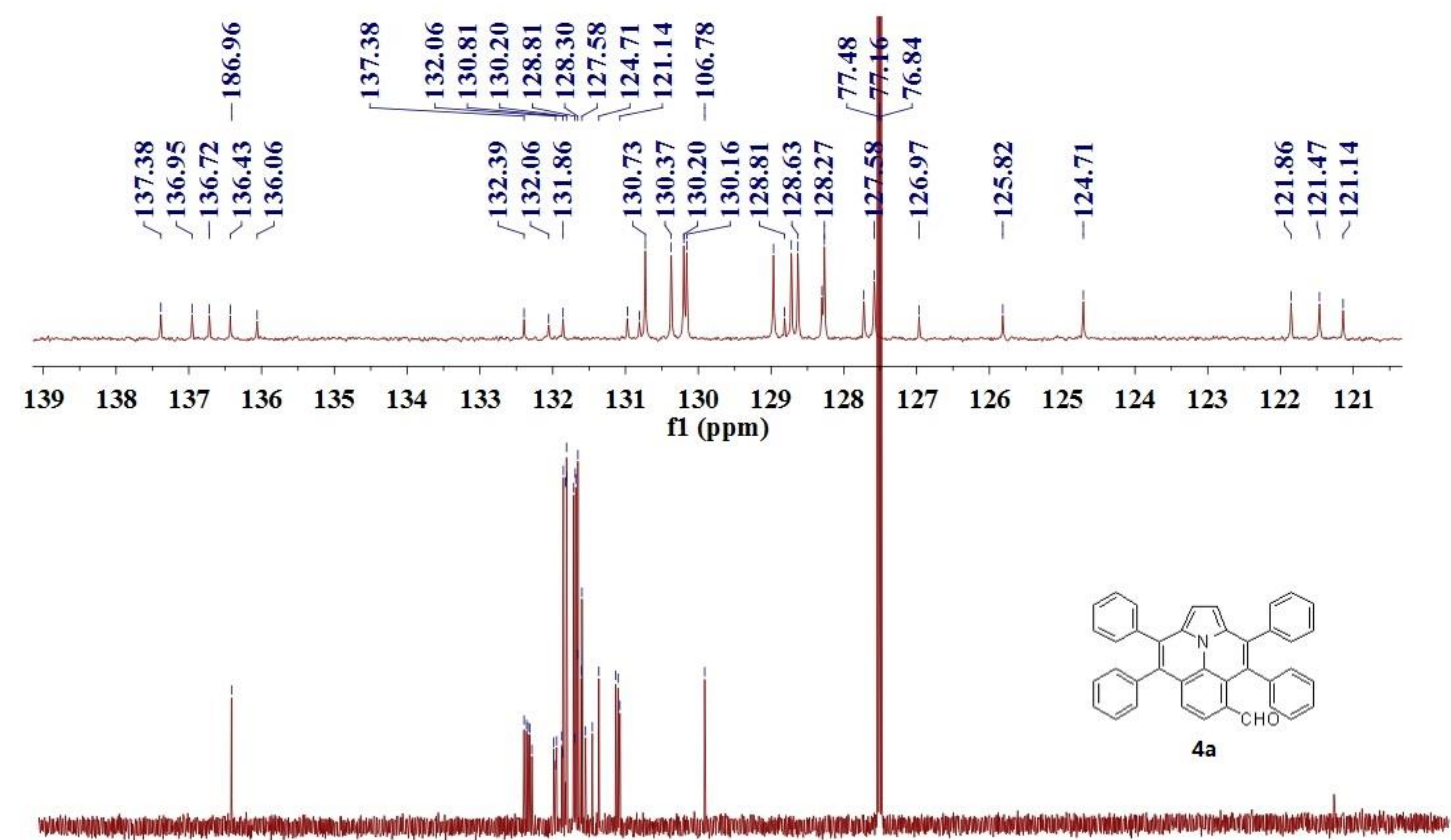

\begin{tabular}{lllllllllllllllll}
\hline 210 & 190 & 170 & 150 & 130 & 110 & 90 & 80 & 70 & 60 & 50 & 40 & 30 & 20 & 10 & 0 &
\end{tabular}

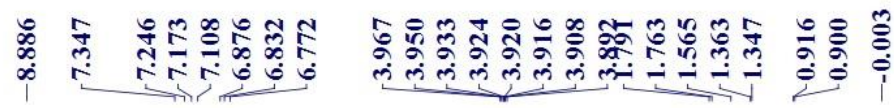

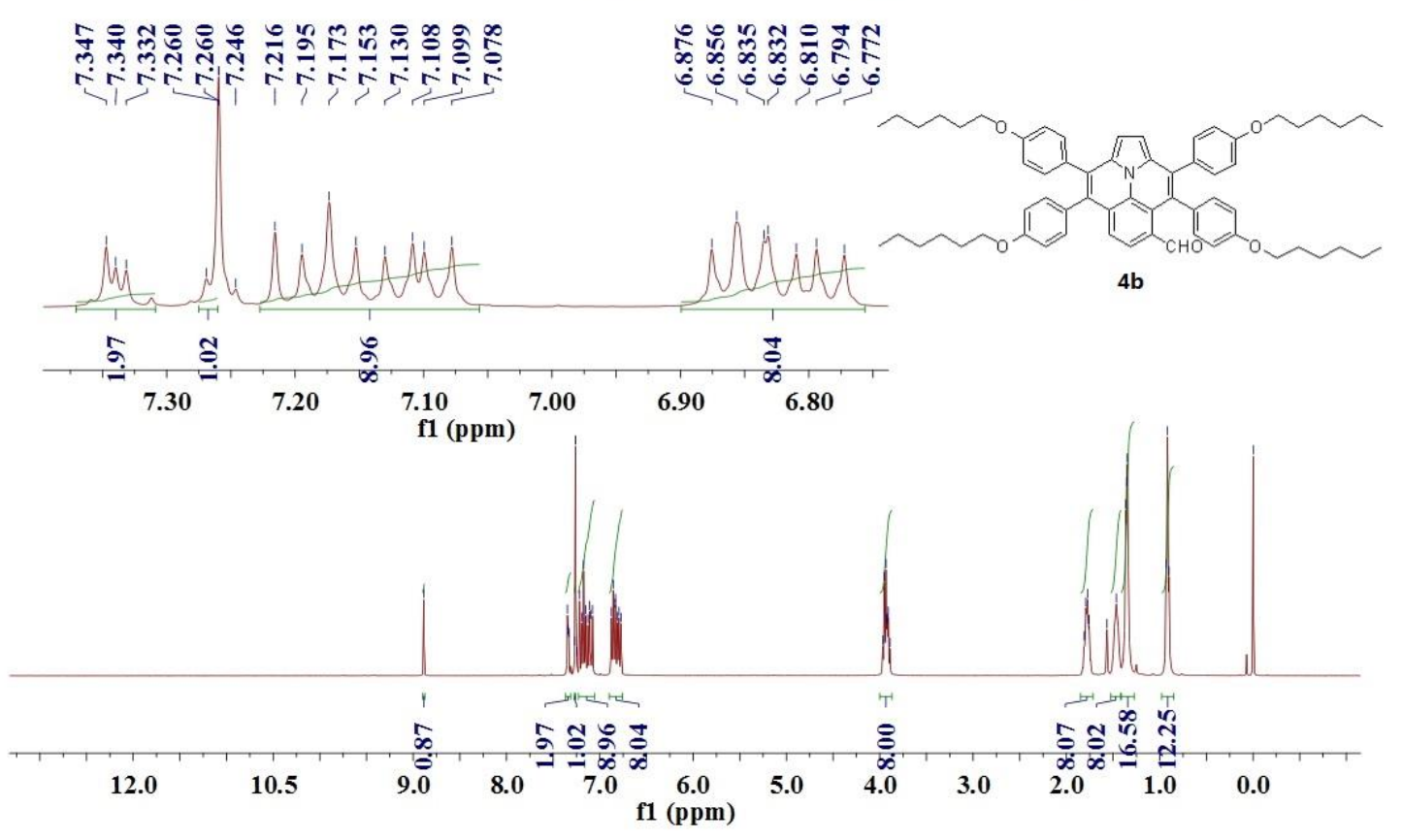




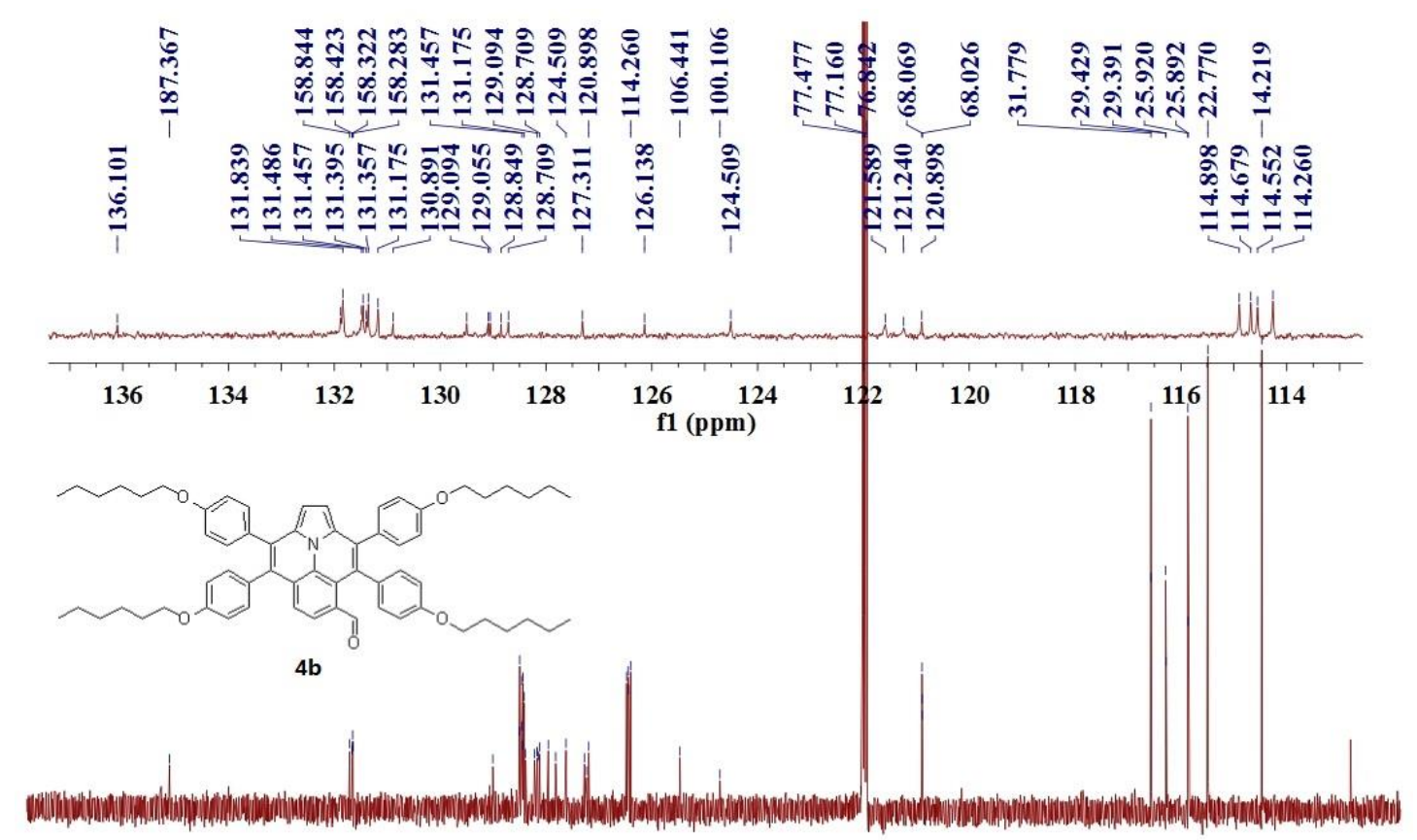

\begin{tabular}{rllllllllllllllllll}
\hline 190 & 170 & 150 & 130 & 110 & 90 & 80 & 70 & 60 & 50 & 40 & 30 & 20 & 10 & 0 &
\end{tabular}

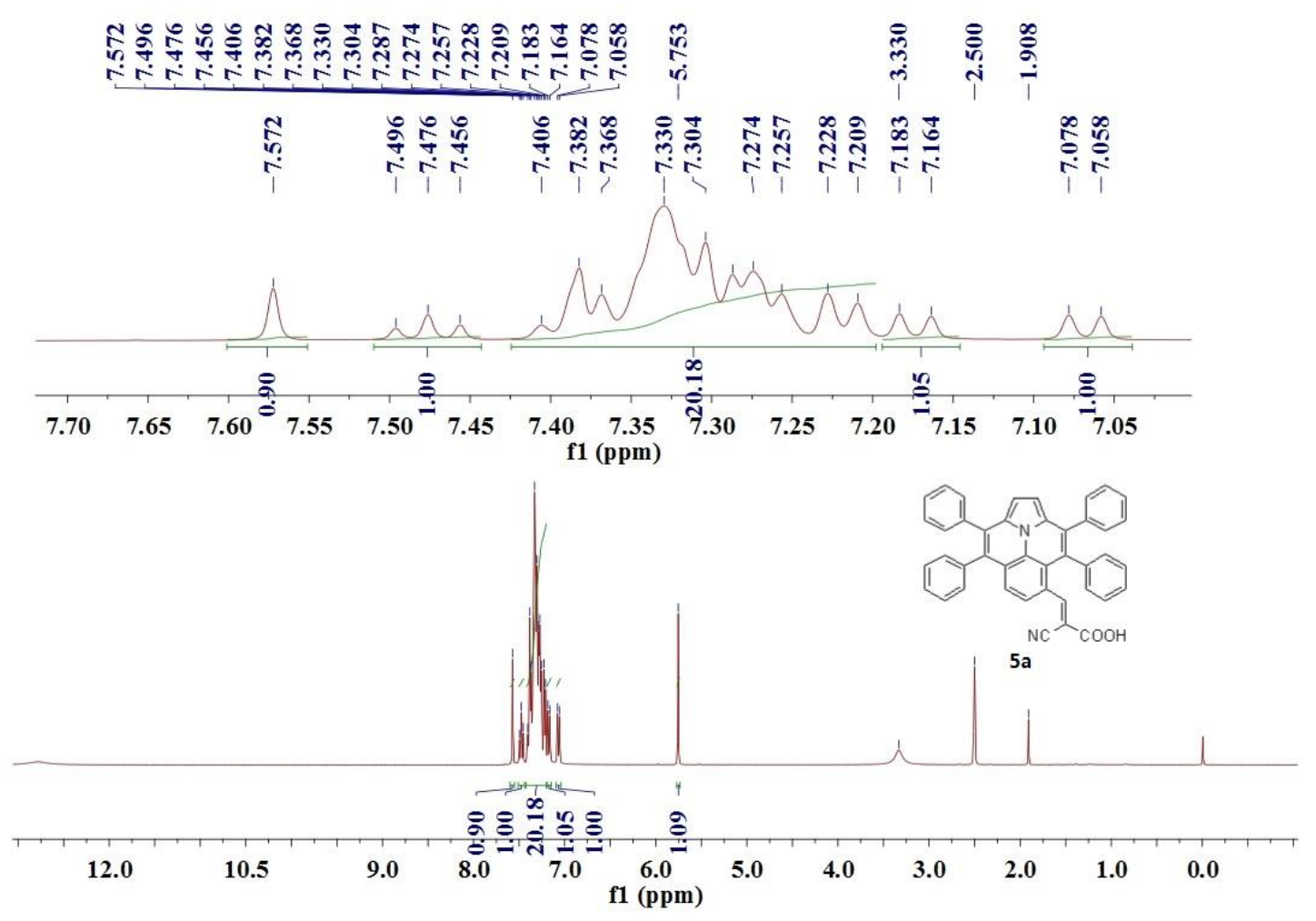




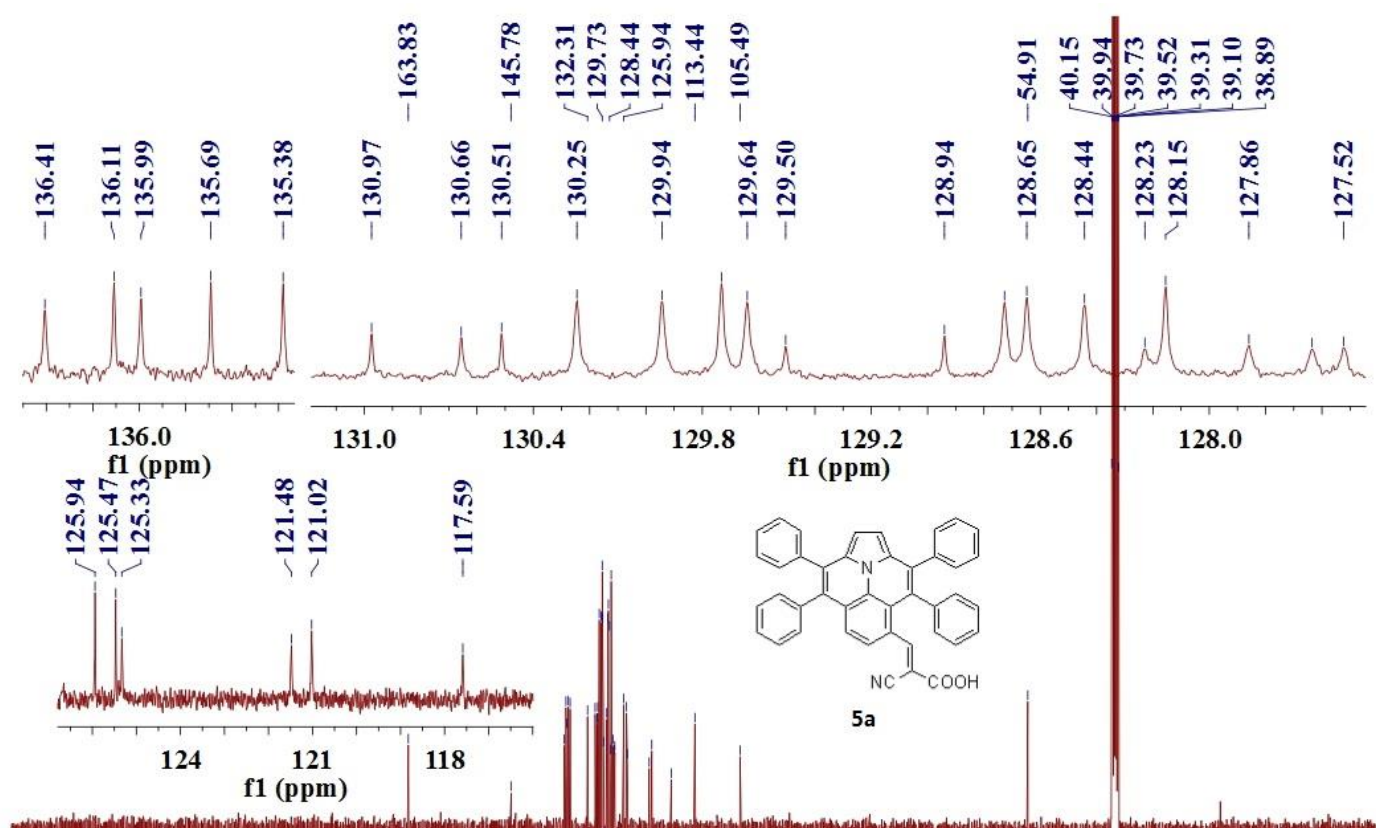

\begin{tabular}{|c|c|c|c|c|c|c|c|c|c|c|c|}
\hline 220 & 200 & 180 & 160 & 140 & $\begin{array}{r}120 \\
\text { f1 }\end{array}$ & $\begin{array}{l}100 \\
\mathrm{~m})\end{array}$ & 80 & 60 & 40 & 20 & 0 \\
\hline
\end{tabular}

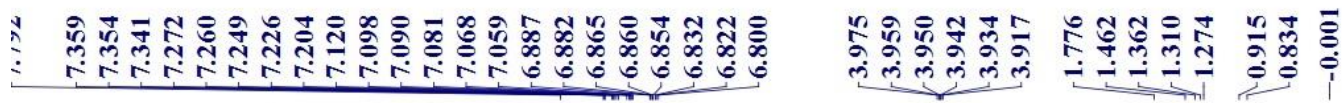

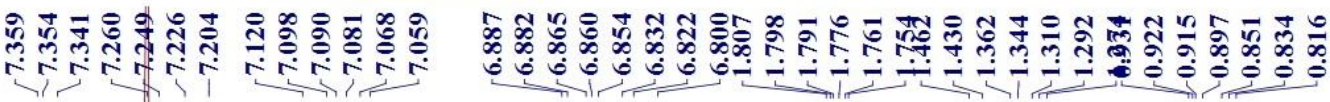
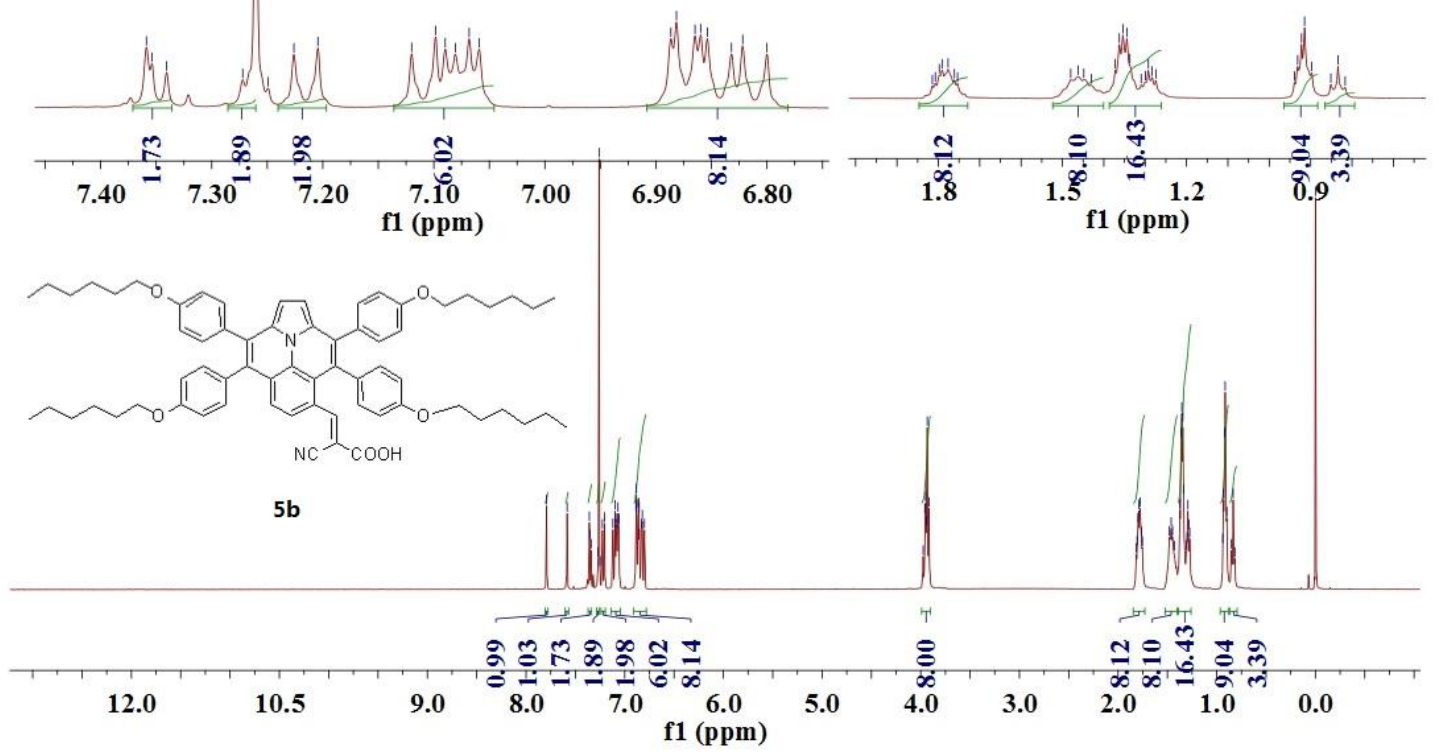

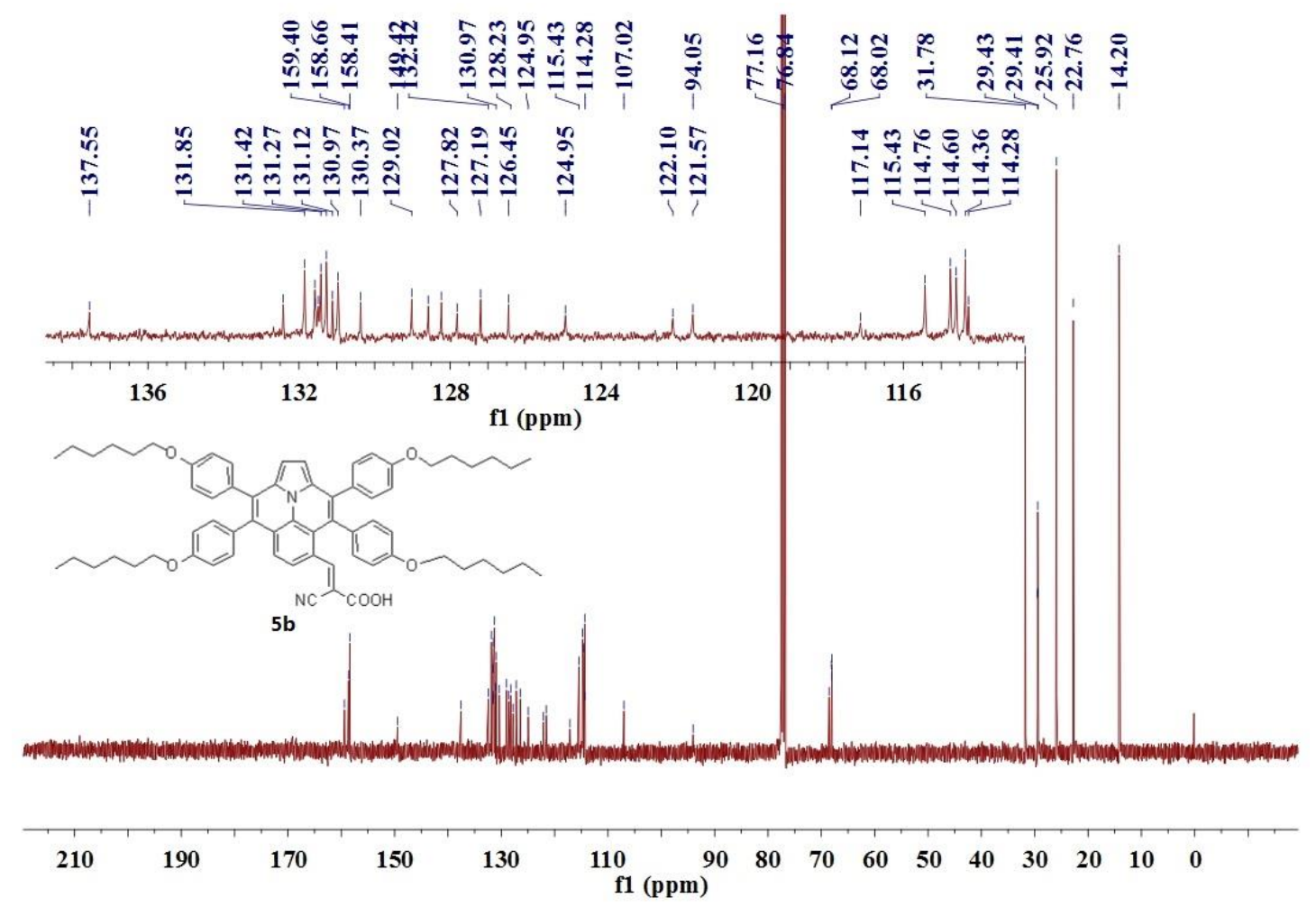

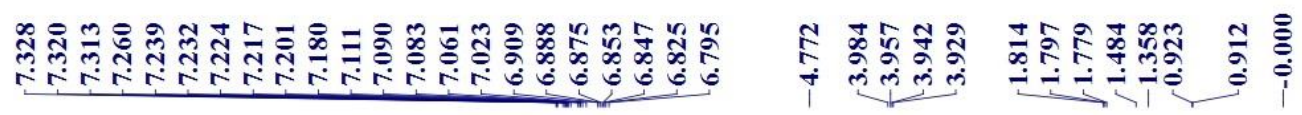

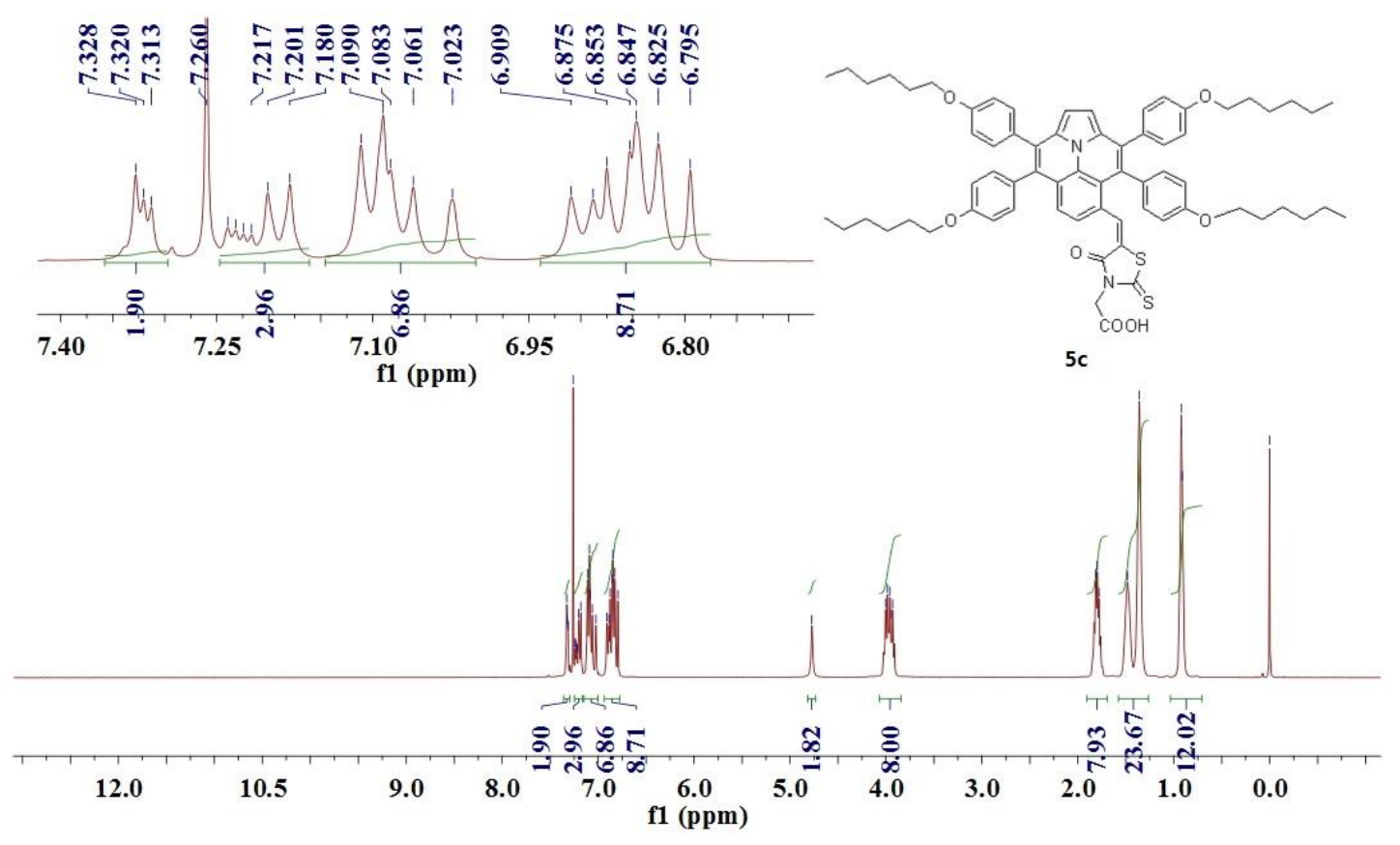



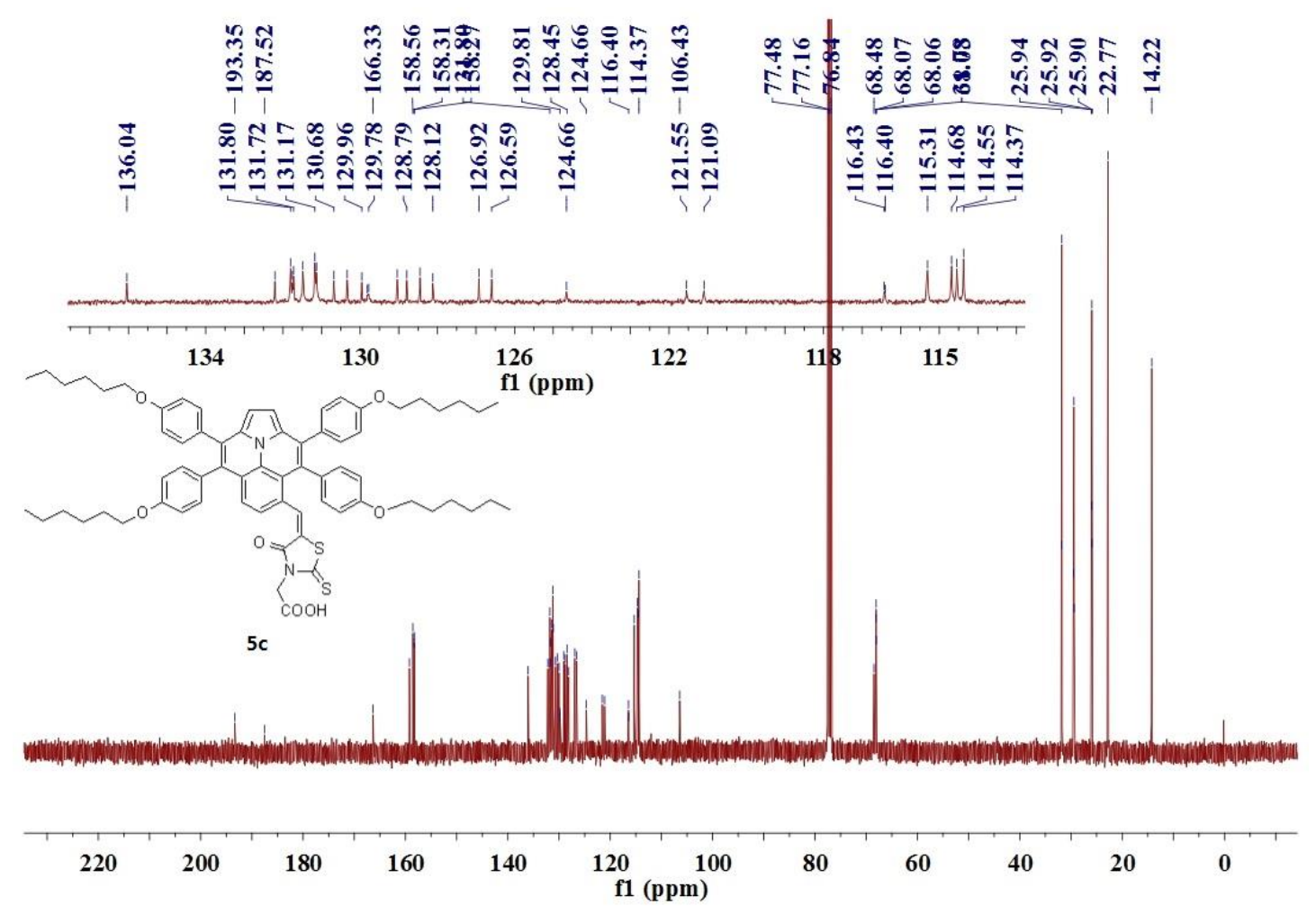